\title{
Measurement of inclusive two-particle angular correlations in pp collisions with the ATLAS detector at the LHC
}

\section{The ATLAS collaboration}

E-mail: atlas.publications@cern.ch

ABSTRACT: We present a measurement of two-particle angular correlations in protonproton collisions at $\sqrt{s}=900 \mathrm{GeV}$ and $7 \mathrm{TeV}$. The collision events were collected during 2009 and 2010 with the ATLAS detector at the Large Hadron Collider using a single-arm minimum bias trigger. Correlations are measured for charged particles produced in the kinematic range of transverse momentum $p_{\mathrm{T}}>100 \mathrm{MeV}$ and pseudorapidity $|\eta|<2.5$. A complex structure in pseudorapidity and azimuth is observed at both collision energies. Results are compared to PYTHIA 8 and HERWIG++ as well as to the AMBT2B, DW and Perugia 2011 tunes of PYTHIA 6. The data are not satisfactorily described by any of these models.

KEYwORDS: Hadron-Hadron Scattering 


\section{Contents}

1 Introduction 1

2 Definition of the two-particle correlation 2

3 The ATLAS detector $\quad 4$

4 Monte Carlo models $\quad 5$

$\begin{array}{lll}5 & \text { Data samples and event selection } & 7\end{array}$

$\begin{array}{llr}6 & \text { Correction procedure } & 8\end{array}$

$\begin{array}{lll}7 & \text { Statistical and systematic uncertainties } & 11\end{array}$

$\begin{array}{lll}7.1 & \text { Statistical uncertainty } & 11\end{array}$

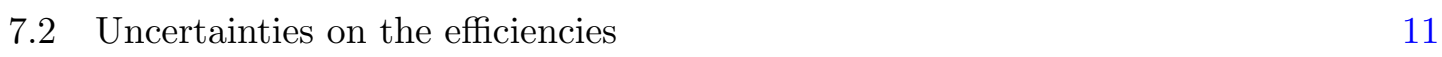

7.3 Uncertainty due to lack of closure using Monte Carlo samples 11

8 Results and discussion $\quad 13$

$\begin{array}{lll}8.1 & \text { Projections in } \Delta \eta \text { and } \Delta \phi & 14\end{array}$

9 Summary and conclusions $\quad 20$

The ATLAS collaboration $\quad 24$

\section{Introduction}

Several phenomenological models have been formulated in order to describe the dynamics of multi-particle production in high-energy hadron collisions. Examples include the string [1] and cluster [2] models, but all have proven to be incomplete since they provide only limited explanations for the observed production of soft particles. The study of correlations between final-state particles is a powerful method for investigating the underlying mechanisms of particle production, as demonstrated by previous experiments [3-5]. Phenomenological models of soft particle production are encapsulated in a variety of different Monte Carlo (MC) generators. Particle correlation measurements provide useful input for discriminating between such models and for tuning their parameters in order to better describe non-perturbative physics. Model features that are sensitive to measurements of correlations include fragmentation, resonance decays, multi-parton interactions, and the emergence of jets. The measurement of correlated particle production in proton-proton collisions is also very useful for studies of heavy ion collisions; any deviation in the latter from the behaviour seen in the former can be attributed to the fact that partons experience 
additional interactions, and other collective effects, in the hot and dense medium present in heavy ion collisions.

This study focuses on the two-particle angular correlations in pseudorapidity, $\eta$, and azimuthal angle, $\phi$, using proton-proton collision data collected by the ATLAS experiment ${ }^{1}$ at the Large Hadron Collider (LHC). A measurement of the inclusive two-particle angular correlation function is performed for charged particles produced in the kinematic region defined by $p_{\mathrm{T}}>100 \mathrm{MeV}^{2}$ and $|\eta|<2.5$ in proton-proton collisions at centre-of-mass collision energies of $\sqrt{s}=900 \mathrm{GeV}$ and $7 \mathrm{TeV}$. For the $7 \mathrm{TeV}$ centre-of-mass energy, an additional region of phase space is considered for which the charged-particle multiplicity per event is at least twenty.

The paper is organised as follows: the two-particle correlation definition used here is described in section 2, while a brief description of the ATLAS detector is presented in section 3. Section 4 summarises the different Monte Carlo models used in this study and the data samples and event selection are described in section 5. In section 6 the data-driven procedure for unfolding the effect of the detection apparatus from the physics is presented. The sources of systematic uncertainty affecting the analysis are discussed in section 7 . Finally, the corrected two-particle angular correlation functions and a comparison to a variety of different Monte Carlo predictions are shown in section 8.

\section{Definition of the two-particle correlation}

The correlation function is defined using two different distributions that are dependent on $\eta$ and $\phi$. The foreground distribution, $F\left(n_{c h}, \Delta \eta, \Delta \phi\right)$, describes the angular separation in azimuth $(\Delta \phi)$ and pseudorapidity $(\Delta \eta)$ between pairs of particles emitted in the same event of charged multiplicity $n_{c h} . F\left(n_{c h}, \Delta \eta, \Delta \phi\right)$ is defined for all events with $n_{c h}$ greater than one and contains correlations due to both the underlying physics processes and detector effects such as geometrical acceptance. The expression for $F\left(n_{c h}, \Delta \eta, \Delta \phi\right)$ is given by

$$
F\left(n_{c h}, \Delta \eta, \Delta \phi\right)=\left\langle\frac{2}{n_{c h}\left(n_{c h}-1\right)} \sum_{i} \sum_{j \neq i} \delta\left(\eta_{i}-\eta_{j}-\Delta \eta\right) \delta\left(\phi_{i}-\phi_{j}-\Delta \phi\right)\right\rangle,
$$

where the summation is over all charged particles in a single event and the average is taken over an ensemble of many events, all having the same charged-particle multiplicity of $n_{c h}$. The delta functions, $\delta\left(\eta_{i}-\eta_{j}-\Delta \eta\right)$ and $\delta\left(\phi_{i}-\phi_{j}-\Delta \phi\right)$, select particle pairs with the $\Delta \eta$ and $\Delta \phi$ separation that is appropriate for $F\left(n_{c h}, \Delta \eta, \Delta \phi\right)$. The total number of chargedparticle pairs in an event is $n_{c h}\left(n_{c h}-1\right) / 2$, therefore if the factor of $2 / n_{c h}\left(n_{c h}-1\right)$ were not included then a particle in a low multiplicity event would carry a lower weight than an

\footnotetext{
${ }^{1}$ ATLAS uses a right-handed coordinate system with its origin at the nominal interaction point (IP) in the centre of the detector and the $z$-axis along the beam pipe. The $x$-axis points from the IP to the centre of the LHC ring, and the $y$-axis points upwards. Cylindrical coordinates $(r, \phi)$ are used in the transverse plane, $\phi$ being the azimuthal angle around the beam pipe. The pseudorapidity is defined in terms of the polar angle $\theta$ as $\eta=-\ln \tan (\theta / 2)$.

${ }^{2}$ The quantity $p_{\mathrm{T}}$ corresponds to the component of the charged-particle momentum transverse to the beam direction.
} 
otherwise identical particle in a higher multiplicity event because there are fewer possible pair combinations.

Since the average charged-particle distribution, $\mathrm{d} n / \mathrm{d} \eta$, is approximately flat in $\eta$ within the acceptance of the measurement, phase-space alone dictates that the $\Delta \eta$ distribution is peaked at $\Delta \eta=0$ and falls approximately linearly to a maximum possible value of $\Delta \eta= \pm 2 \eta_{\max }$. This motivates the second distribution used in constructing the correlation function, the background correlation $B$, the $\Delta \eta$ dependence of which at a fixed chargedparticle multiplicity is given by

$$
\begin{aligned}
B\left(n_{c h}, \Delta \eta\right)= & \int_{-\eta_{\max }}^{+\eta_{\max }} \int_{-\eta_{\max }}^{+\eta_{\max }} \mathrm{d} \eta_{1} \mathrm{~d} \eta_{2} \delta\left(\eta_{1}-\eta_{2}-\Delta \eta\right) \\
& \times\left.\left.\frac{1}{n_{c h}} \frac{\mathrm{d} n\left(n_{c h}, \eta\right)}{\mathrm{d} \eta}\right|_{\eta=\eta_{1}} \frac{1}{n_{c h}} \frac{\mathrm{d} n\left(n_{c h}, \eta\right)}{\mathrm{d} \eta}\right|_{\eta=\eta_{2}},
\end{aligned}
$$

where $\mathrm{d} n\left(n_{c h}, \eta\right) / \mathrm{d} \eta$ is the $\eta$ distribution of charged particles in events of fixed chargedparticle multiplicity $n_{c h}$ and the factors of $1 / n_{c h}$ ensure that the integral of $B$ over all $\Delta \eta$ is unity. The function $\delta\left(\eta_{1}-\eta_{2}-\Delta \eta\right)$ is a Dirac delta function. The $\Delta \phi$ dependence of $B$ is defined analogously to, and is flatter than, the $\Delta \eta$ dependence.

The correlation function $C\left(n_{c h}, \Delta \eta, \Delta \phi\right)$ can be constructed using the foreground and background distributions, and is

$$
C\left(n_{c h}, \Delta \eta, \Delta \phi\right)=F\left(n_{c h}, \Delta \eta, \Delta \phi\right)-B\left(n_{c h}, \Delta \eta, \Delta \phi\right) .
$$

Previous analyses [3] have found that $\left(n_{c h}-1\right) \times C\left(n_{c h}, \Delta \eta, \Delta \phi\right)$ is approximately independent of $n_{c h}$. By multiplying equation (2.3) by a factor of $\left(n_{c h}-1\right)$ and averaging the resulting quantity over all charged particle multiplicities greater than one, we obtain the multiplicity-independent result, $\bar{C}$, given by

$$
\begin{aligned}
\bar{C}(\Delta \eta, \Delta \phi) & =\sum_{n_{c h}} P\left(n_{c h}\right)\left(n_{c h}-1\right)\left\{F\left(n_{c h}, \Delta \eta, \Delta \phi\right)-B\left(n_{c h}, \Delta \eta, \Delta \phi\right)\right\} \\
& =\left\langle\left(n_{c h}-1\right) F\left(n_{c h}, \Delta \eta, \Delta \phi\right)\right\rangle_{c h}-\left\langle\left(n_{c h}-1\right) B\left(n_{c h}, \Delta \eta, \Delta \phi\right)\right\rangle_{c h},
\end{aligned}
$$

where $P\left(n_{c h}\right)$ is the probability density function for $n_{c h}$ and the notation $\langle\ldots\rangle_{c h}$ indicates that a weighted average over contributions from all charged-particle multiplicities should be made.

Dividing equation (2.4) by the multiplicity-averaged background distribution then gives the multiplicity-independent two-particle correlation function

$$
R(\Delta \eta, \Delta \phi)=\frac{\left\langle\left(n_{c h}-1\right) F\left(n_{c h}, \Delta \eta, \Delta \phi\right)\right\rangle_{c h}}{\left\langle B\left(n_{c h}, \Delta \eta, \Delta \phi\right)\right\rangle_{c h}}-\frac{\left\langle\left(n_{c h}-1\right) B\left(n_{c h}, \Delta \eta, \Delta \phi\right)\right\rangle_{c h}}{\left\langle B\left(n_{c h}, \Delta \eta, \Delta \phi\right)\right\rangle_{c h}} .
$$

Since the $\mathrm{d} n / \mathrm{d} \eta$ distribution, and hence the background, is not strongly dependent on the charged particle multiplicity, the factor of $B$ approximately cancels from the second term on the right of equation (2.5) with two caveats. First, the background does have some multiplicity dependence at low multiplicity due to the contribution from double diffraction, which favours higher $\Delta \eta$ values compared to the non-diffractive contribution. Second, the 
effect of any track reconstruction inefficiency is larger in low multiplicity events compared to high multiplicity events because a small number of mis-reconstructed tracks has a proportionally larger significance in the former. As an example, consider a low multiplicity event with a small number of correlated track clusters; the loss of a small fraction of tracks could easily remove completely one of the track clusters, thus changing the overall correlation. The loss of the same fraction of tracks in a higher multiplicity event is less likely to remove such a feature; it will instead simply reduce the overall number of track pairs. Calculating a multiplicity independent background distribution, $B(\Delta \eta, \Delta \phi)$, using $\langle\mathrm{d} n / \mathrm{d} \eta\rangle_{c h}$, which is already averaged over all multiplicities, therefore has the advantage of diluting the effect of the experimentally more troublesome lower multiplicity events. Such an approach slightly reduces the sensitivity of the observable to diffraction. However, diffraction is not the motivation for this measurement. The final expression used for the inclusive two-particle correlation function is then given by

$$
R(\Delta \eta, \Delta \phi)=\frac{\left\langle\left(n_{c h}-1\right) F\left(n_{c h}, \Delta \eta, \Delta \phi\right)\right\rangle_{c h}}{B(\Delta \eta, \Delta \phi)}-\left\langle n_{c h}-1\right\rangle_{c h} .
$$

In practice, the expression $\left\langle\left(n_{c h}-1\right) F\left(n_{c h}, \Delta \eta, \Delta \phi\right)\right\rangle_{c h}$ is constructed by taking each pair of particles within a single event, calculating their absolute $\eta$ and $\phi$ separations and filling one quadrant of a two-dimensional distribution at those values using a weight of $2 / n_{c h}$. The other three quadrants are filled by reflection, making the distribution symmetric around $(0,0)$. This distribution is normalised by dividing each bin by the number of events entering the distribution. The background is determined by taking random pairs of events and, for each particle in one event, the $|\Delta \eta|$ and $|\Delta \phi|$ values with each particle in the other event are calculated and used to fill another two-dimensional distribution, in the same way as done to the foreground distribution, which is then normalised to unit integral.

Projections of the two-dimensional correlation function along both $\Delta \eta$ and $\Delta \phi$ help reveal more details of the structure of the correlations and permit easier comparisons with different models. These projections are calculated by first integrating separately the foreground and the background distributions before taking the ratio between the two, and normalising with the average track multiplicity.

\section{The ATLAS detector}

The ATLAS detector [6] is one of the two general-purpose detectors at the LHC [7]. Collision events are reconstructed by layers of tracking, calorimeter and muon systems covering almost the whole solid angle around the collision point. For the analysis presented in this paper, the tracking detectors and trigger systems are the most relevant.

At the centre of ATLAS lies the inner detector (ID), which is responsible for reconstructing the trajectories and measuring the transverse momenta of charged particles and reconstructing the vertices from which they originate. The ID has complete azimuthal coverage and consists of three sub-systems, which cover the pseudorapidity range of $|\eta|<2.5$. The three ID sub-systems are: a silicon pixel detector, a semiconductor tracking detector $(\mathrm{SCT})$ and a transition radiation tracker (TRT). They are immersed in a $2 \mathrm{~T}$ axial 
magnetic field and cover a radial distance from the nominal interaction point in the barrel of 50.5-150 mm, 299-560 $\mathrm{mm}$ and 563-1066 mm, respectively. The ID sensors provide a position resolution of 10,17 and $130 \mu \mathrm{m}$ for the $r-\phi$ coordinate and of 115 and $580 \mu \mathrm{m}$ for the second coordinate in the case of the pixel and SCT detectors. The angular resolution of the tracks reconstructed from the pixel, SCT and TRT hits is between $40 \mu \mathrm{rad}$ (for $\eta \simeq 0.25$ ) and $50 \mu \mathrm{rad}$ (for $\eta \simeq 1.75$ ) in azimuth and between 5 and $10 \mathrm{in} \cot (\theta)$.

There are three levels in the ATLAS trigger system: Level-1, Level-2 and the Event Filter. The first level uses limited information to perform an initial selection of potentially interesting events, with a fixed latency of $2.5 \mu \mathrm{s}$. The subsequent levels use full granularity measurements to further reduce the selected events to a level acceptable for offline reconstruction. For this measurement, only the Level-1 trigger signals from the beam pickup timing devices (BPTX) and minimum bias trigger scintillators (MBTS) are used to select candidate events. The BPTX electrostatic button pick-up detectors are attached to the beam-pipe and located at $\pm 175 \mathrm{~m}$ from the collision point. The MBTS consists of plastic scintillators on the inner edge of the calorimeter end-caps, which lie on both sides of the detector, each at a distance of $3.56 \mathrm{~m}$ from the interaction point. Segmented into eight sectors in azimuth, they have a pseudorapidity coverage of $2.09<|\eta|<2.82$ and $2.82<|\eta|<3.84$. A coincidence of the BPTX signals between the two sides of ATLAS (i.e. colliding bunches) and at least one hit above threshold from either side of the MBTS (referred to as single-arm trigger) are required.

\section{Monte Carlo models}

In order to compare the present measurement to different soft QCD models, three MC event generators are considered: PYTHIA 6.4 (versions 6.421 and 6.425) [8], PYTHIA 8.150 [9] and HERWIG++ version 2.5.1 [10].

The PYTHIA approach to soft interactions uses phenomenological adaptations of the lowest-order perturbative QCD to describe low- $p_{\mathrm{T}}$ scattering processes in order to extend its physics description to the non-perturbative regime. HERWIG ++ , on the other hand, extends its eikonal concept of independent multi-partonic scatters down into the infrared region [11]. Other differences between HERWIG++ and PYTHIA include the hadronisation model and the ordering of the parton shower. PYTHIA uses a hadronisation model based upon colour strings and a $p_{\mathrm{T}}$ or virtuality-ordered shower, while HERWIG ++ implements a cluster hadronisation scheme and a shower that is ordered in emission angle.

Each Monte Carlo generator provides a set of parameters whose values can be altered to produce different MC tunes. Several tunes of PYTHIA version 6.4 are compared to data: AMBT2B [12], Perugia 2011 [13] and DW [14]. Version 6.425 of PYTHIA was used in all cases apart from tune DW at $900 \mathrm{GeV}$, for which version 6.421 was used. ${ }^{3}$ Tune $4 \mathrm{C}$ of PYTHIA 8.150 [15] as well as the UE7-2 underlying-event tune at $\sqrt{s}=7 \mathrm{TeV}$ and the MU900-2 minimum bias tune at $\sqrt{s}=900 \mathrm{GeV}$ [16] of HERWIG $++[17]$ are also used for comparison.

\footnotetext{
${ }^{3}$ The small differences between the minor PYTHIA versions do not affect the non-perturbative physics relevant for this measurement.
} 
AMBT2B is a tune of PYTHIA 6 that was produced by the ATLAS Collaboration as a result of optimising earlier tunes to best describe ATLAS results. In particular, parameters controlling the initial state radiation (ISR) cut-off and evolution, plus further parameters that adjust the model for multi-parton interactions (MPI) were modified to reproduce data on jet shapes and minimum bias events from ATLAS. The tune AMBT2B with the CTEQ6L1 parton density functions (PDFs) [18] is used here, and provides one of the best available descriptions of existing minimum bias data.

The Perugia series of tunes predates the availability of LHC data; however, the most recent Perugia 2011 tune has been adjusted to take account of measurements made at the LHC. Differences between the Perugia 2011 and AMBT2B tunes include the ISR evolution and the parameters controlling the reconnection of outgoing string fragments to the beam remnants. Perugia 2011 is shown here with the CTEQ 5L PDFs.

Both AMBT2B and Perugia 2011 use a $p_{\mathrm{T}}$-ordered parton shower and a model in which multi-parton interactions are interleaved with ISR. DW, on the other hand, is a tune that uses a virtuality-ordered parton shower and a MPI model that is not interleaved with the initial state shower. It is optimised to describe underlying event and Drell-Yan data from the Tevatron.

PYTHIA 8 adds to the MPI model of PYTHIA 6 by interleaving not only the ISR, but also the final state radiation (FSR) with the MPI. PYTHIA 8 includes an updated model of diffraction that includes harder colour singlet exchange; compared to PYTHIA 6 , this allows for harder $p_{\mathrm{T}}$ and particle multiplicity distributions from the single- and double-diffractive contributions. Like the version of AMBT2B shown here, tune $4 \mathrm{C}$ of PYTHIA 8 uses the CTEQ6L1 PDFs. While PYTHIA 8 has not yet been tuned to data as extensively as PYTHIA 6 , tune $4 \mathrm{C}$ was the result of comparisons to early LHC data after PYTHIA 8 had already been tuned to give reasonable agreement with Tevatron minimum bias data. The result is that tune $4 \mathrm{C}$ has a reduced diffractive contribution and more realistic average $p_{\mathrm{T}}$ per particle compared to earlier tunes of PYTHIA 8.

HERWIG++ is used here with the MRST2007LO* PDFs [19]. The current version of HERWIG ++ does not have a model for diffraction, so it has been tuned to diffractionsuppressed LHC data. HERWIG++ has a model for hadronisation that is based on cluster decay, rather than the string fragmentation used by PYTHIA. It is therefore interesting to test HERWIG ++ against the present measurement in order to compare the two hadronisation models and to ascertain the importance of a diffractive model in describing two-particle correlations.

In addition to these models, the older Perugia 0 [20] and ATLAS MC09 [21] PYTHIA 6 tunes, the Tune 1 of PYTHIA 8.130 and the PHOJET [22] generator are used for systematic studies.

Samples containing non-diffractive and diffractive components, mixed according to the generator cross sections, are used when appropriate for those models that provide a separate diffractive contribution. Particles with a mean decay length $(c \tau)$ greater than $10 \mathrm{~mm}$ are not decayed by the generator. As a consequence, secondary particles (such as the decay products of neutral kaons) are excluded from the generator-level distributions. This setting also defines the hadronic final state to which the measured distributions are corrected. 
The generated events are processed through the GEANT 4 [23] based ATLAS detector simulation program [24]. In addition to simulating the detector, GEANT 4 also handles the decays of particles whose mean decay length is longer than $10 \mathrm{~mm}$. Having been fully simulated, MC events are then reconstructed and analysed in the same manner as the data.

\section{Data samples and event selection}

This study uses the same selection criteria as the charged-particle multiplicity analysis carried out at $\sqrt{s}=900 \mathrm{GeV}$ and $7 \mathrm{TeV}$ [25]. In total, approximately $7 \mu \mathrm{b}^{-1}$ of $900 \mathrm{GeV}$ data and $190 \mu \mathrm{b}^{-1}$ of $7 \mathrm{TeV}$ data are analysed. The events were collected during 2009 and 2010 using low instantaneous luminosity proton beams such that the mean number of interactions per bunch crossing was around 0.01. Only events in which the inner detector was fully operational and the solenoidal magnet was on are used.

Tracks are selected for analysis if they have:

- $p_{\mathrm{T}}>100 \mathrm{MeV}$,

- $|\eta|<2.5$

- at least 1 hit in the innermost layer of the pixel detector,

- at least 1 hit in any of the layers of the pixel detector,

- at least 2, 4 or 6 hits in the SCT for tracks with $p_{\mathrm{T}}>100,200$ and $300 \mathrm{MeV}$, respectively,

- a transverse impact parameter with respect to the primary vertex, $d_{0}^{\mathrm{PV}}$, of $\left|d_{0}^{\mathrm{PV}}\right|<$ $1.5 \mathrm{~mm}$,

- a longitudinal impact parameter with respect to the primary vertex, $z_{0}^{\mathrm{PV}}$, of $\left|z_{0}^{\mathrm{PV}} \sin \theta\right|<1.5 \mathrm{~mm}$ (where $\theta$ is the angle between the track and the $z$-axis),

- a track-fit $\chi^{2}$ probability of at least 0.01 for those tracks with $p_{\mathrm{T}}>10 \mathrm{GeV}$ (to remove tracks with mis-measured $p_{\mathrm{T}}$ due to misalignment or interactions within the detector material).

To reduce the contribution from background events (such as beam-halo, beam-gas interactions and cosmic rays) and non-primary tracks, events are required:

- to have been triggered by the single-arm trigger,

- to contain at least one primary vertex. Primary vertices are required to be associated with at least two tracks with $p_{\mathrm{T}}>100 \mathrm{MeV}$ with a transverse impact parameter with respect to the beam spot ${ }^{4}$ of $\left|d_{0}^{\mathrm{BS}}\right|<4 \mathrm{~mm}$,

- not to have a second interaction vertex associated to more than four tracks (to remove events with more than one proton-proton interaction in a single bunch crossing),

\footnotetext{
${ }^{4}$ The beam spot is defined as the centre of the luminous region in which the proton beams interact.
} 


\begin{tabular}{|l|c|c|c|}
\hline Phase-Space Region & $\sqrt{s}[\mathrm{TeV}]$ & Selected Events & Selected Tracks \\
\hline$n_{c h} \geq 2, p_{\mathrm{T}}>100 \mathrm{MeV},|\eta|<2.5$ & 0.9 & 357,523 & $4,532,663$ \\
\cline { 2 - 4 } & 7 & $10,066,072$ & $209,809,430$ \\
\hline$n_{c h} \geq 20, p_{\mathrm{T}}>100 \mathrm{MeV},|\eta|<2.5$ & 7 & $4,029,565$ & $153,553,766$ \\
\hline
\end{tabular}

Table 1. Total number of selected events and tracks in $900 \mathrm{GeV}$ and $7 \mathrm{TeV}$ data.

- to contain a minimum number of tracks, depending on the phase-space region, passing the track selection criteria previously described.

For data taken at $\sqrt{s}=7 \mathrm{TeV}$, two different phase-space regions with varying contributions from diffractive events are explored: $n_{c h} \geq 2$ and $n_{c h} \geq 20$. For data taken at $\sqrt{s}=900 \mathrm{GeV}$, only the particle multiplicity requirement of $n_{c h} \geq 2$ is used. The total numbers of selected events and tracks are shown in table 1.

\section{Correction procedure}

In order to disentangle the effects caused by the apparatus and the reconstruction algorithms from the true physics processes, the following corrections are applied to the reconstructed data.

First, the foreground and multiplicity distributions are weighted with the inverse of the trigger and vertex reconstruction efficiencies. These efficiencies, taken directly from ref. [25], are calculated before a requirement on the vertex is made. The efficiencies were determined in ref. [25] from data by using a trigger independent of the Level 1 MBTS (for the trigger efficiency) and by relaxing the requirement on the presence of a reconstructed vertex (for the vertex efficiency). These efficiencies depend on the track multiplicity, where the total number of selected tracks is obtained by applying all of the selection criteria described in section 5 apart from the $d_{0}^{\mathrm{PV}}$ and $z_{0}^{\mathrm{PV}} \sin \theta$ requirements. A requirement on the transverse impact parameter with respect to the beam spot position, $d_{0}^{\mathrm{BS}}$, is made instead such that $\left|d_{0}^{\mathrm{BS}}\right|<1.8 \mathrm{~mm}$. The trigger efficiency is determined using a test trigger that selects events from random proton bunch crossings and uses information from the pixel and SCT detectors at Level 2.

The effect of tracks lost due to inefficiencies in the reconstruction is corrected for using the model-independent Hit Backspace Once More (HBOM) method described in ref. [26]. Figure 1 illustrates this correction procedure, in which the observable is first computed using all reconstructed tracks that satisfy the analysis selection criteria (solid circles in figure 1a). The track reconstruction efficiency as a function of the track $p_{\mathrm{T}}$ and $\eta$ is taken from ref. [25]. For each track, a random number, $r_{i}$, uniformly distributed between zero and one, is generated and compared to the track reconstruction efficiency, $\epsilon_{i}\left(p_{\mathrm{T}}, \eta\right)$. A track is removed from the sample if the random number is greater than the track reconstruction efficiency. This results in a subset of the original tracks, defined by all those tracks satisfying

$$
\epsilon_{i}\left(p_{\mathrm{T}}, \eta\right)>r_{i}
$$




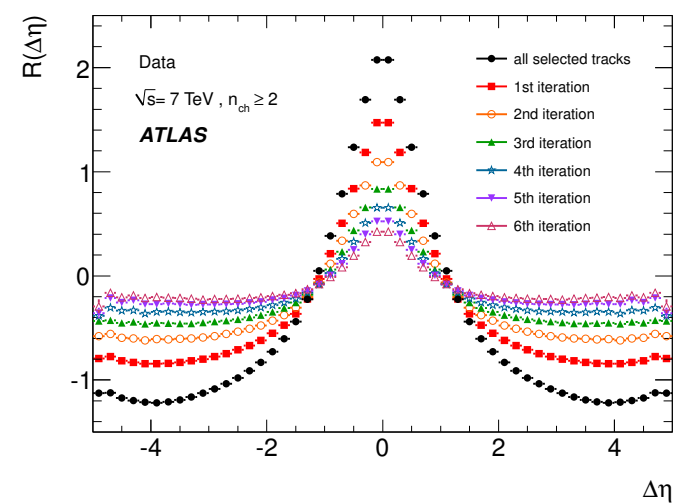

(a)

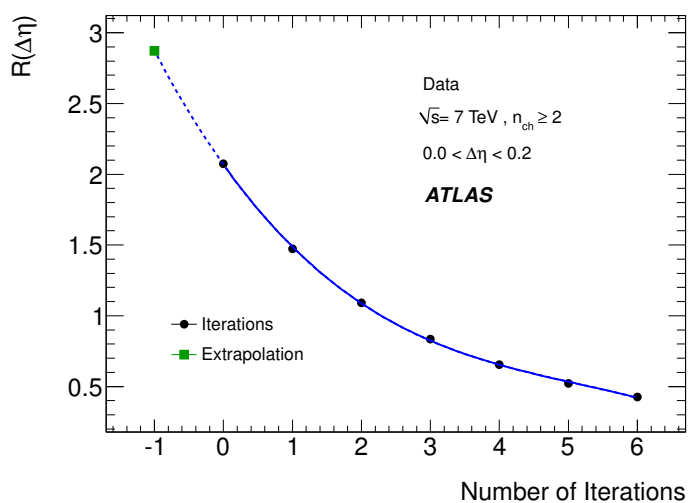

(b)

Figure 1. (a) Two-particle pseudorapidity correlation function, $R(\Delta \eta)$, after each iteration of the probabilistic track removal correction method. (b) Example of a third-degree polynomial fit to the values of $R(\Delta \eta)$ in a central bin. Plots of $R(\Delta \eta)$ shown here and throughout this paper are symmetric around $\Delta \eta=0$ by construction.

Tracks removed from the sample in this way are lost with the same probability as they would be lost by the detector reconstruction. The reduced sample of tracks, which now expresses an exaggerated detector reconstruction effect, is used to compute the observable again. This defines one iteration of the track-removal procedure (the full set of uncorrected tracks is the 0th iteration). A second iteration takes as input the tracks used in the first iteration and again uses a new set of random numbers to remove additional tracks according to their track reconstruction efficiency. The observable is then determined from this third sample of tracks that shows a larger detector effect than the first two samples. Further iterations are carried out in the same way.

Using this probabilistic track removal method, it is possible to quantify the effect that detector inefficiencies have on an observable; each iteration corresponds to an additional application of the detector effect to the data. The value of each bin of the observable can be plotted as a function of the iteration number $(0,1,2, \ldots, N)$ and a function can be fitted to the resulting set of points. By extrapolating the fit to the $N=-1$ iteration, an estimate of the observable at the particle level, without detector effects, can be made. In this analysis the fit and subsequent extrapolation use a third-degree polynomial, with six track removal iterations in total used as the input, as illustrated in figure $1 \mathrm{~b}$ for one particular $\Delta \eta$ bin.

No specific correction is made for tracks arising from the secondary decays of longer lived particles nor from material interactions. The effect of secondary particles is discussed in section 7.3.

In order to test the correction procedure, Monte Carlo studies are performed in which the distributions at the generated particle level are recovered from a fully detector-simulated and reconstructed Monte Carlo sample. This is shown in figure 2 for the PYTHIA 6 MC09 tune at $\sqrt{s}=900 \mathrm{GeV}$ and $7 \mathrm{TeV}$ for $R(\Delta \eta)$ with $0<\Delta \phi<\pi$. The size of the correction that must be applied to the uncorrected correlation function depends on the value of $\Delta \eta$ and $\Delta \phi$; in general the correction has an absolute value of around 0.5. At high $|\Delta \eta|$ the 


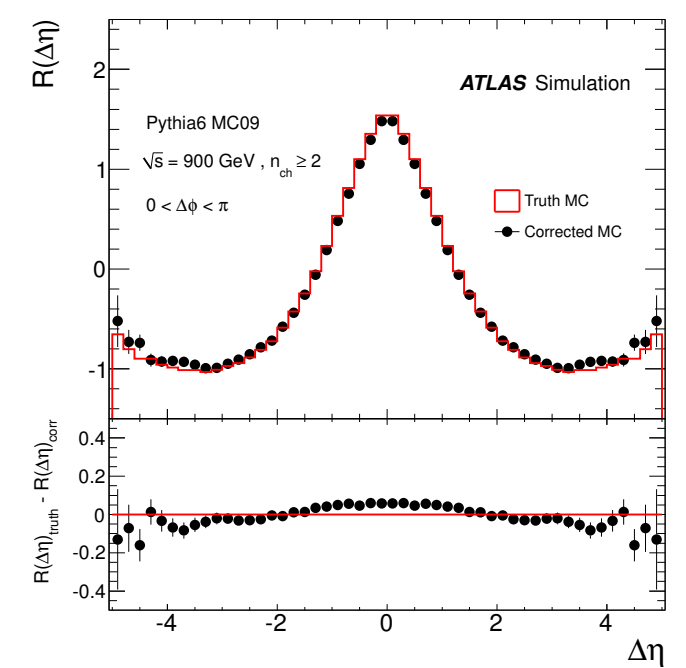

(a)

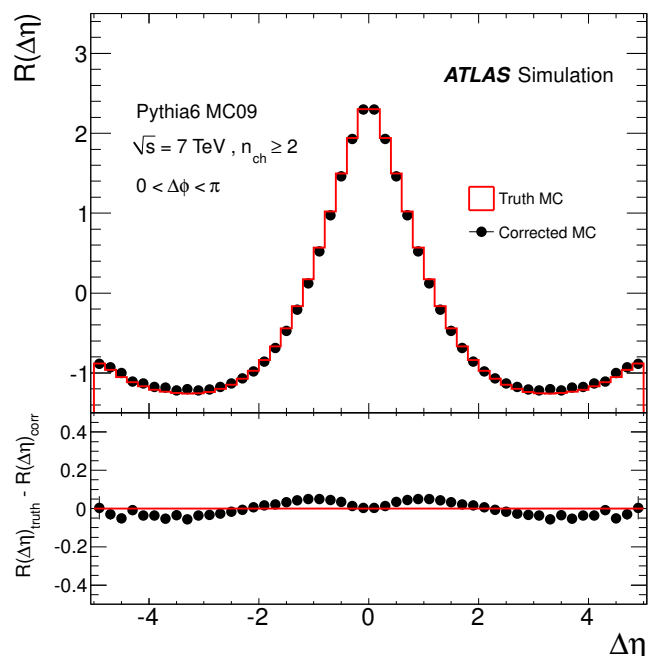

(b)

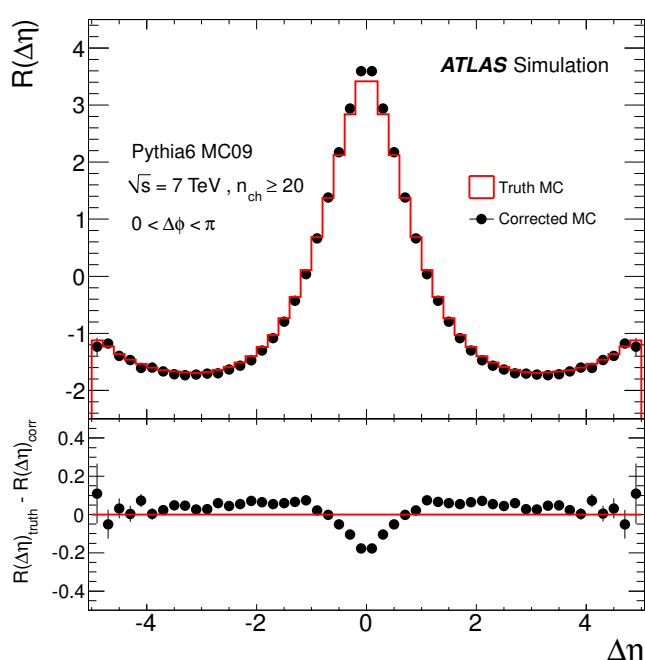

(c)

Figure 2. Comparison between the generated and corrected two-particle correlation functions $R(\Delta \eta)$ at (a) $\sqrt{s}=900 \mathrm{GeV}$ and $7 \mathrm{TeV}$ for the phase-space regions (b) $n_{c h} \geq 2$ and (c) $n_{c h} \geq 20$ for the Monte Carlo MC09 tune. The absolute difference between the generated and corrected MC as a function of $\Delta \eta$ is shown underneath each distribution.

correction is negative, while at low $|\Delta \eta|$ the correction is positive, so the overall effect is that the peak at $\Delta \eta=0$ is narrowed significantly. The generated and corrected distributions are generally in good agreement with each other, showing an absolute difference of approximately 0.05 in all but a few bins. The remaining differences are considered as a systematic uncertainty, as discussed in section 7.3.

All the distributions presented in this paper, both one- and two-dimensional, have been corrected using this procedure. No correction is made for the effects of angular smearing because the resolution of the tracking detector is much better than the bin size used. Any such correction would be insignificant in comparison to the uncertainty on the efficiency correction that is applied. 


\section{Statistical and systematic uncertainties}

The sources of uncertainty identified for this measurement are described in this section. The most significant contributions to both the one- and two-dimensional distributions are associated with the track reconstruction efficiency and with the correction procedure (arising from the small differences between the generated and corrected Monte Carlo distributions).

\subsection{Statistical uncertainty}

Each of the parameters in the third-degree polynomial fits in the probabilistic track removal method carries a statistical error. The statistical uncertainty in the corrected values is the result of propagating these uncertainties, using the full covariance matrix, when evaluating the third-degree equation at $N=-1$. In general, this uncertainty is negligible compared to the ones associated to the track reconstruction efficiency and the non-closure of the correction method. The exceptions to this are the highest $\Delta \eta$ bins where, due to reduced statistics, the uncertainty on the fit parameters is of the same magnitude as the other uncertainties.

\subsection{Uncertainties on the efficiencies}

In order to evaluate the systematic uncertainty that is associated with uncertainties in the trigger, vertex and track reconstruction efficiencies, the complete analysis is repeated varying each of these quantities, one at a time, according to their uncertainties. It is assumed there is no difference in the size of these uncertainties between the different collision energies, as discussed in ref. [25].

The systematic uncertainties associated with the track reconstruction efficiency come from various sources: material effects, track selection, momentum resolution and badly measured high- $p_{\mathrm{T}}$ tracks. The tracking efficiency is obtained in ref. [25] by matching MC generated charged particles to tracks reconstructed after the full detector simulation. Uncertainties on the efficiency are determined by varying the parameters of the detector simulation. These uncertainties are summarised in table 2. All of these uncertainties are added in quadrature to obtain the total uncertainty on the tracking efficiency in each $\left(p_{\mathrm{T}}, \eta\right)$ bin.

In the case of the trigger and vertex reconstruction efficiencies, the systematic uncertainties are of the order of $1 \%$ for events with a track multiplicity of two and decrease rapidly as the multiplicity increases.

\subsection{Uncertainty due to lack of closure using Monte Carlo samples}

The absolute difference between the generated and corrected Monte Carlo distributions is used as the systematic uncertainty resulting from the small non-closure of the correction procedure. From this it is possible to derive an estimate for the non-closure uncertainty in data as a function of the corrected value of $R$.

This is illustrated in figure 3 for $R(\Delta \eta$ ) (where $\Delta \phi$ was integrated from 0 to $\pi$ ) for different tunes at $\sqrt{s}=7 \mathrm{TeV}$ for $n_{c h} \geq 2$. The absolute amount of non-closure does not depend strongly on either the Monte Carlo model or on the value of $R(\Delta \eta)$ and, for this 


\begin{tabular}{|l|l|}
\hline Source & Uncertainty \\
\hline Material description in MC & $\begin{array}{l}\text { Increases at high- }|\eta| \text { and decreases at high- } p_{\mathrm{T}} ; \\
\text { max. } 15 \% \text { in the range } 2.3<|\eta|<2.5 \\
\text { and } 100 \mathrm{MeV}<p_{\mathrm{T}}<150 \mathrm{MeV}\end{array}$ \\
\hline Track selection & $1 \%$ in all $\left(p_{\mathrm{T}}, \eta\right)$ bins \\
\hline$p_{\mathrm{T}}$ resolution in MC & $5 \%$ in the first $p_{\mathrm{T}}$ bin $\left(100 \mathrm{MeV}<p_{\mathrm{T}}<150 \mathrm{MeV}\right)$ \\
\hline High- $p_{\mathrm{T}}$ tracks & $10 \%$ due to the requirement on the track-fit $\chi^{2}$ probability \\
\cline { 2 - 2 } & $\begin{array}{l}\eta \text { and } p_{\mathrm{T}} \text { dependent uncertainty due to } \\
\text { mis-measured tracks }\end{array}$ \\
\hline
\end{tabular}

Table 2. Uncertainties associated to the track reconstruction efficiency. Values are taken from and are identical to those in ref. [25], which provides a more complete discussion on this subject.

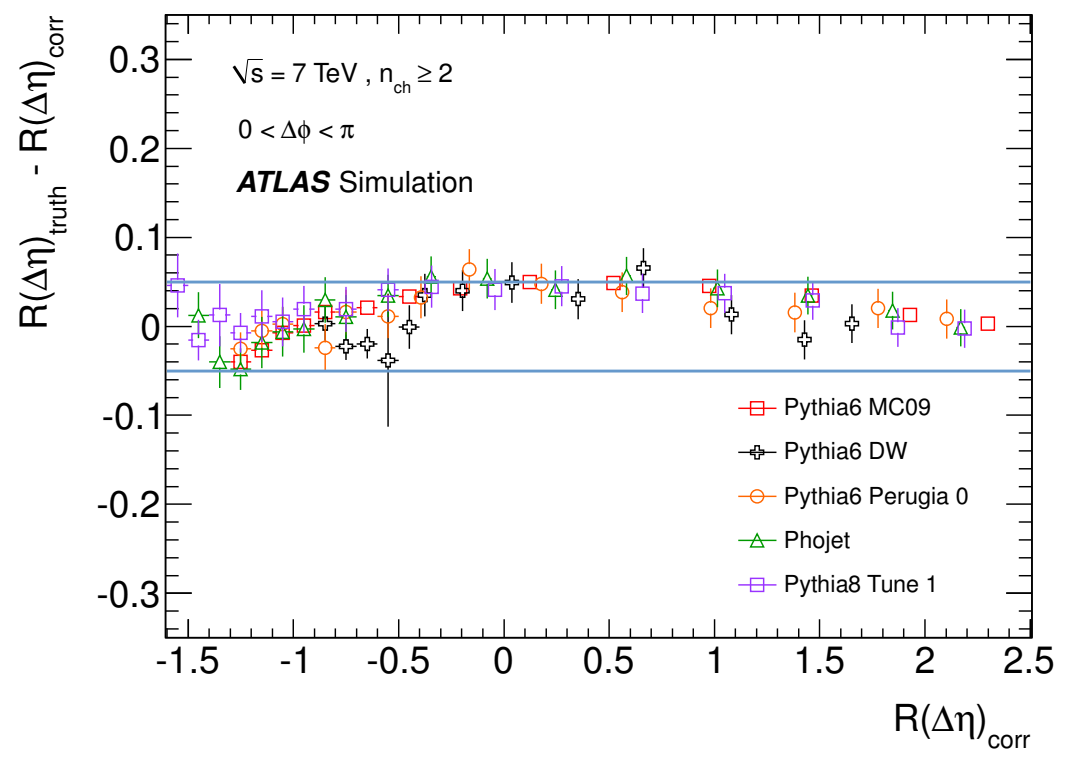

Figure 3. Difference between the generated and corrected two-particle correlation functions as a function of $R(\Delta \eta)_{\text {corr }}$ for various Monte Carlo tunes at $\sqrt{s}=7 \mathrm{TeV}$ for $n_{c h} \geq 2$.

example, lies generally in the region between \pm 0.05 . Therefore in this case a systematic uncertainty of 0.05 is assigned to all bins in the data distribution.

The systematic uncertainty associated with the non-closure of the correction method is calculated in this manner for each of the one-dimensional projections and the twodimensional distributions. In most cases the systematic uncertainty has a value of 0.05 or smaller and has very little dependence on $R$; however, in a small number of cases for some of the $n_{c h} \geq 20$ distributions, the uncertainty can be as large as 0.2 .

The small non-closure of the corrected samples with the hadron-level Monte Carlo distributions is primarily caused by the introduction of additional particles in the detector 
simulation that are not produced by the Monte Carlo generator. Such secondary particles can be produced either by the decays of longer-lived particles that are set stable by the generator (primarily neutral kaons), or by interactions between primary particles and the detector material. Both sources of secondary particles produce correlated pairs of tracks, and as such contribute to the non-closure in the same manner; however, the pairs of secondary particles initiated by detector interactions produce an effect that is between five and ten times as large as the effect produced by neutral decay products.

Using the reconstruction efficiency to remove tracks from the data sample provides an approximation to the effect that the detector has on the observable. This approximation does not account for the effect of fake tracks or of tracks that arise from secondary decays and material interactions. In order to apply a correction for these small effects, the probabilities that such particles are produced would need to be assessed so that they could be used to add additional particles probabilistically in each iteration of the correction procedure. Studies using the full detector simulation show that the size of such corrections would be small in comparison to the correction for tracking inefficiencies and would be of a similar size to other systematic uncertainties present in this measurement. This justifies the approach of applying a correction for tracking inefficiencies, which is the largest detector effect, and assigning a non-closure systematic based on studies using the full detector simulation.

\section{Results and discussion}

The corrected inclusive two-particle angular correlation functions for data and the PYTHIA 6 AMBT2B Monte Carlo tune are shown in figures 4 and 5 for $\sqrt{s}=900 \mathrm{GeV}$ and $7 \mathrm{TeV}$, respectively.

At both energies a complex structure can be observed across the full $\Delta \eta$ and $\Delta \phi$ range. Several components can be identified that reflect the contributions from different underlying processes to the correlation structure. Most obvious are the near-side correlations, which are seen as a sharp peak around $(0,0)$ that can be attributed to particles originating from the same (relatively) high- $p_{\mathrm{T}}$ process. Such closely correlated particles indicate the emergence of jet-like structures within this minimum bias event selection. Another prominent structure in the two-dimensional distribution is the away-side correlation, which is seen as a ridge extending across the whole of the $\Delta \eta$ range near $\Delta \phi \sim \pi$. This away-side activity arises from the recoil of one particle against another and would be seen quite strongly in, for example, back-to-back jet production. A third distinct structure is the broad Gaussian-like structure spanning the $\Delta \phi$ domain at $\Delta \eta=0$ with a width of $\Delta \eta \simeq 2$. This last structure can be related to processes such as the decays of resonances, clusters or string fragmentation.

As the centre-of-mass energy increases, so too does the height of the peak at $(0,0)$. This peak becomes even more pronounced for the set of events with a higher charged-particle multiplicity (figure 5c). Conversely, the height of the away-side ridge is constant regardless of the collision energy or multiplicity. PYTHIA 6 AMBT2B exhibits similar structures to those observed in data; however it does not reproduce the strength of the correlations. 


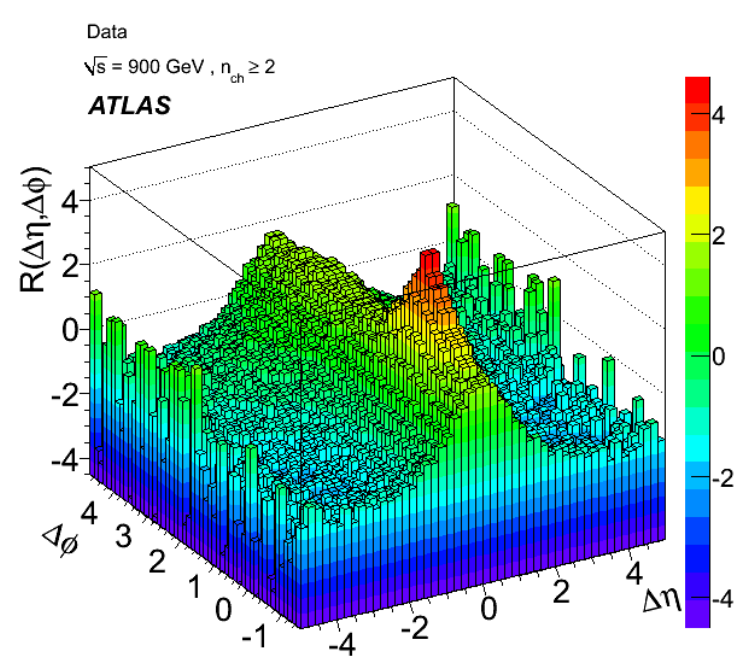

(a)

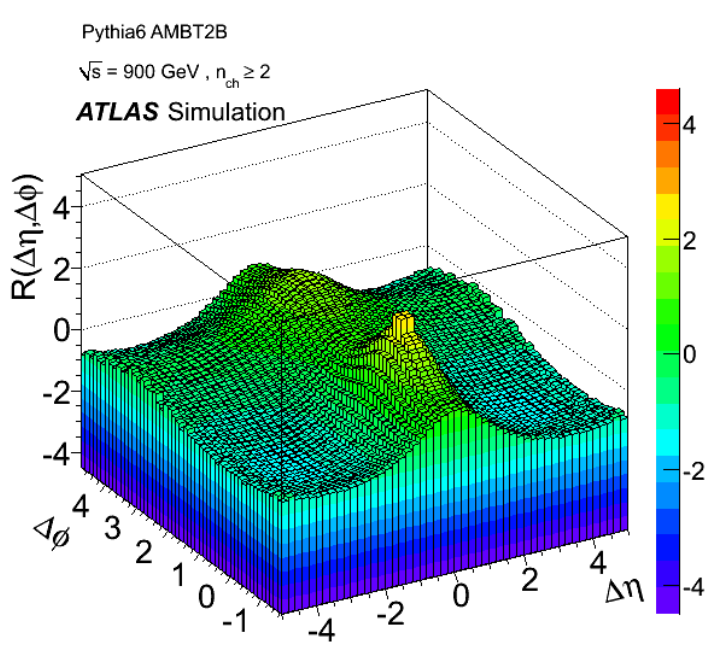

(b)

Figure 4. Corrected $R(\Delta \eta, \Delta \phi)$ two-particle correlation functions for (a) data and (b) the AMBT2B Monte Carlo tune, at $\sqrt{s}=900 \mathrm{GeV}$.

\subsection{Projections in $\Delta \eta$ and $\Delta \phi$}

In all of the following figures, the solid markers correspond to the corrected data values, the error bars are statistical only (after correcting for track detection inefficiency) and the solid bands correspond to the total uncertainty in the bin, which is obtained by adding in quadrature the statistical and systematic uncertainties described in section 7 . The data distributions are compared to different Monte Carlo tunes. In general, none of the models reproduce the strength of the correlations seen in data. Some approximate the shape of the distributions more closely than others, but for all distributions HERWIG ++ is the most discrepant with the data.

The corrected pseudorapidity correlation distributions are shown in figure 6 and were obtained by integrating separately the foreground and background functions over $\Delta \phi$ between 0 and $\pi$. The AMBT2B and Perugia 2011 tunes of PYTHIA 6 together with tune 4C of PYTHIA 8 show the best agreement with the data distributions for events with $n_{c h} \geq 2$ at both collision energies. For the sample of events satisfying $n_{c h} \geq 20$ the AMBT2B tune shows the closest agreement to the data over the entire $\Delta \eta$ range, although the agreement cannot be considered satisfactory.

In order to separate the $\eta$ correlations on the near side $(\Delta \phi \simeq 0)$ from those on the away side $(\Delta \phi \simeq \pi)$, the pseudorapidity correlation function was also calculated by integrating the foreground and background functions over $\Delta \phi$ within the more limited ranges of $\left(0: \frac{\pi}{2}\right)$ (near side) and $\left(\frac{\pi}{2}: \pi\right)$ (away side). Figure 7 shows that, when only the near side is included, the peak at $(0,0)$ becomes narrower, higher and more pronounced, which indicates a stronger correlation between nearby particles. Tune $4 \mathrm{C}$ of PYTHIA 8 provides the best description of the data across the entire $\Delta \eta$ range for the $n_{c h} \geq 2$ event samples at both energies, while the AMBT2B tune provides the most reasonable description of events for which $n_{c h} \geq 20$. 


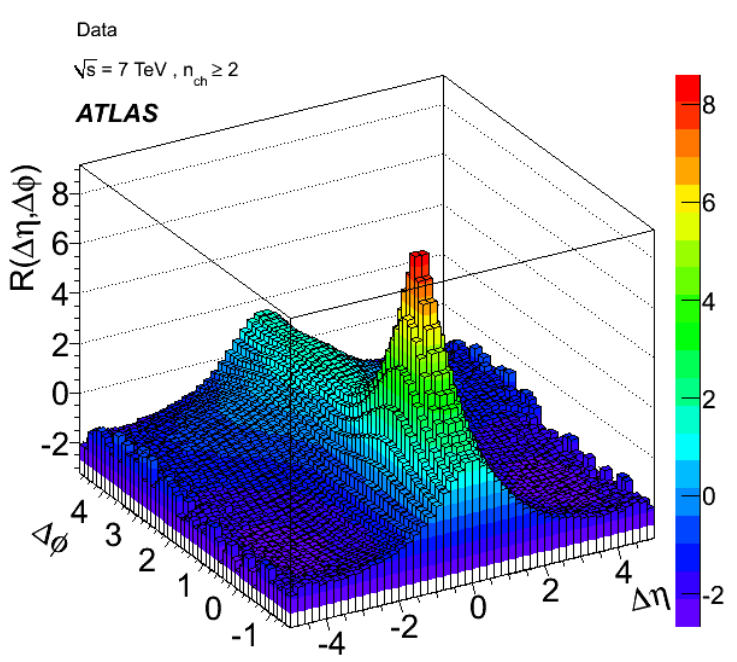

(a)

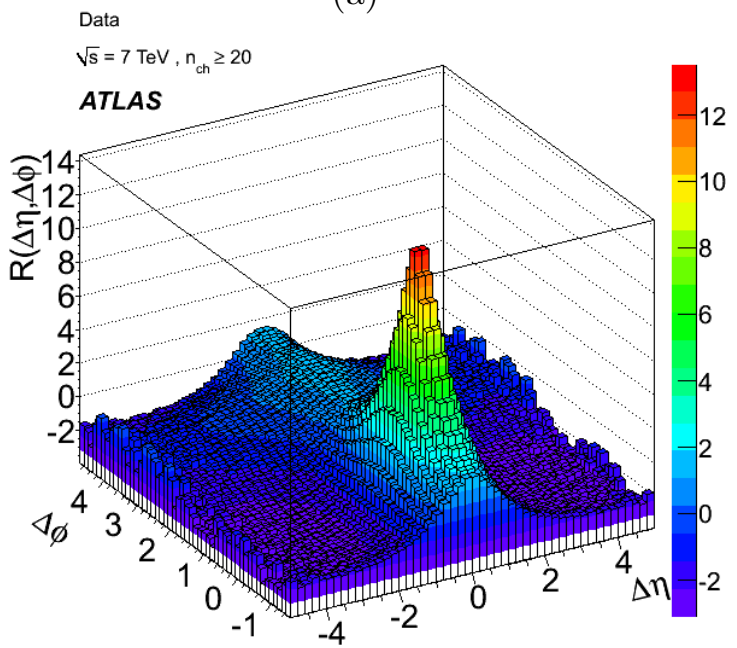

(c)

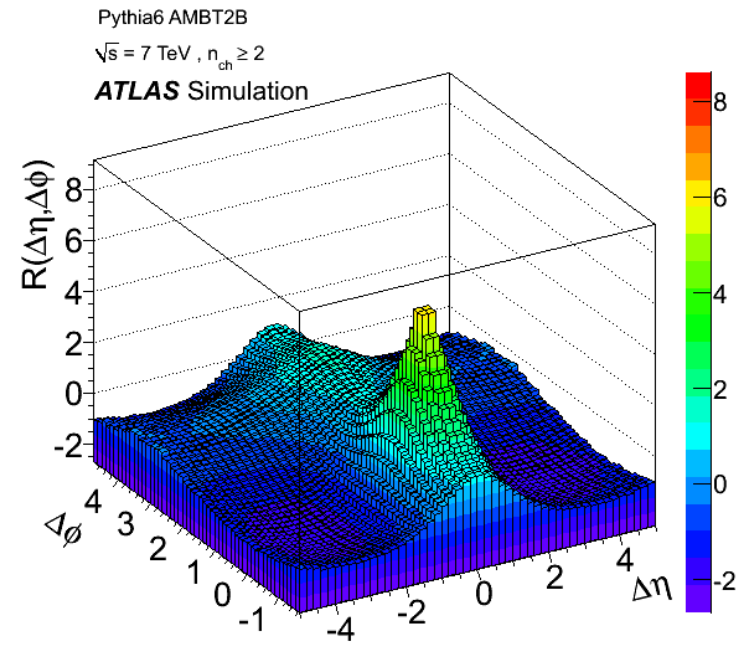

(b)

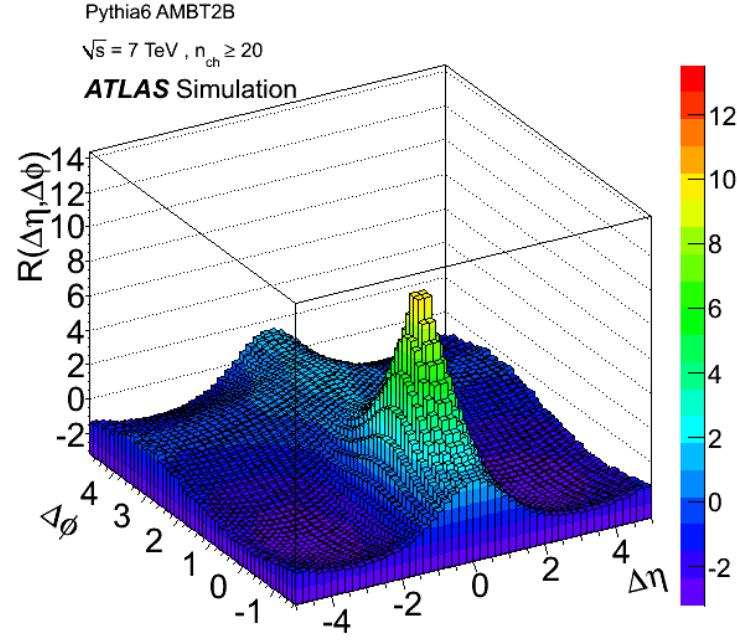

(d)

Figure 5. Corrected $R(\Delta \eta, \Delta \phi)$ two-particle correlation functions at $\sqrt{s}=7 \mathrm{TeV}$ for $n_{c h} \geq 2$ (a) data and (b) Monte Carlo (AMBT2B), and for $n_{c h} \geq 20$ (c) data and (d) Monte Carlo (AMBT2B). These plots are symmetric around $\Delta \eta=0$ by construction.

The $\eta$ correlations on the away side are shown in figure 8 , and here most of the tunes (with the exception of HERWIG++) display a better agreement with data. The Perugia 2011 tune shows a particularly good agreement with data across the $\Delta \eta$ range at both collision energies.

The dependence of the correlation function on the azimuthal separation can similarly be studied by integrating the foreground and background over $\Delta \eta$ for two different ranges: (0:2) (short-range correlations) and (2:5) (long-range correlations). The short-range correlation functions, shown in figure 9 , contain two peaks. The first peak is at $\Delta \phi=0$ and becomes more pronounced if either the collision energy or the particle multiplicity is increased. The second peak is at $\Delta \phi=\pi$ and has a height that is approximately indepen- 

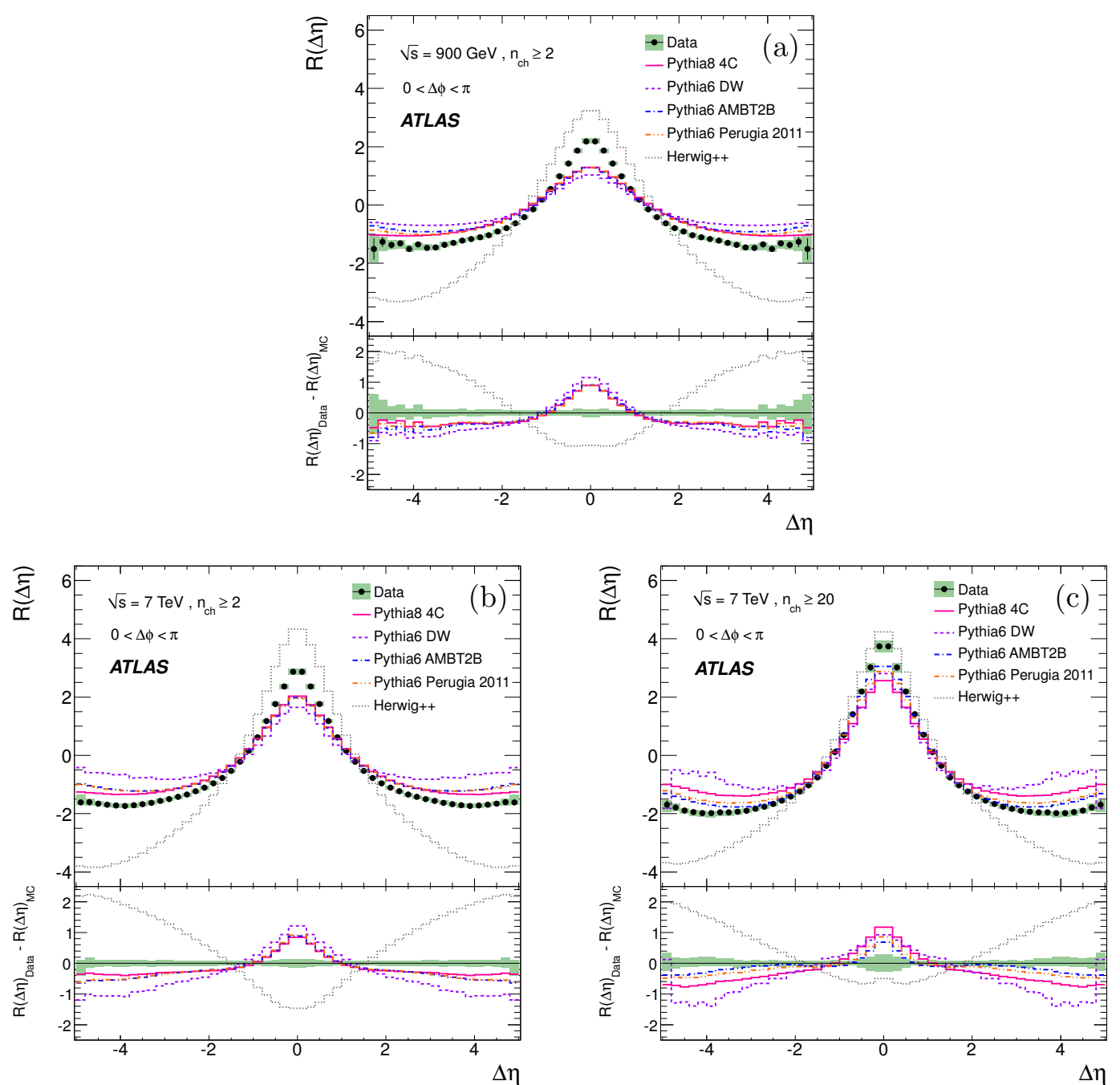

Figure 6. Corrected $R(\Delta \eta)$ two-particle correlation functions obtained by integrating the foreground and background distributions over $\Delta \phi$ between 0 and $\pi$ for data and several different Monte Carlo tunes at (a) $\sqrt{s}=900 \mathrm{GeV}$ and $7 \mathrm{TeV}$ for events with a charged-particle multiplicity (b) $n_{c h} \geq 2$ and (c) $n_{c h} \geq 20$.

dent of both the collision energy and the multiplicity. We note the general similarity in the shape of these functions to some distributions obtained in measurements of underlying event; in particular the trough in the correlation function around $\Delta \phi \simeq \pi / 2$ is somewhat analogous to the "transverse" region of ref. [27].

None of the Monte Carlo models provides a good description of the $\Delta \phi$ dependence of the short-range correlation function across the entire $\Delta \phi$ range. AMBT2B and Perugia 2011 tend to agree with data on the away side, but less so on the near side. DW, which has previously provided reasonable agreement with the transverse region of underlying event data, does not agree particularly well with the correlation function. Tune $4 \mathrm{C}$ of PYTHIA 8 shows good agreement with data in some regions near the two peaks, but a clear disagreement in others, particularly the region around $\Delta \phi \simeq \pi / 2$ at $\sqrt{s}=7 \mathrm{TeV}$. 

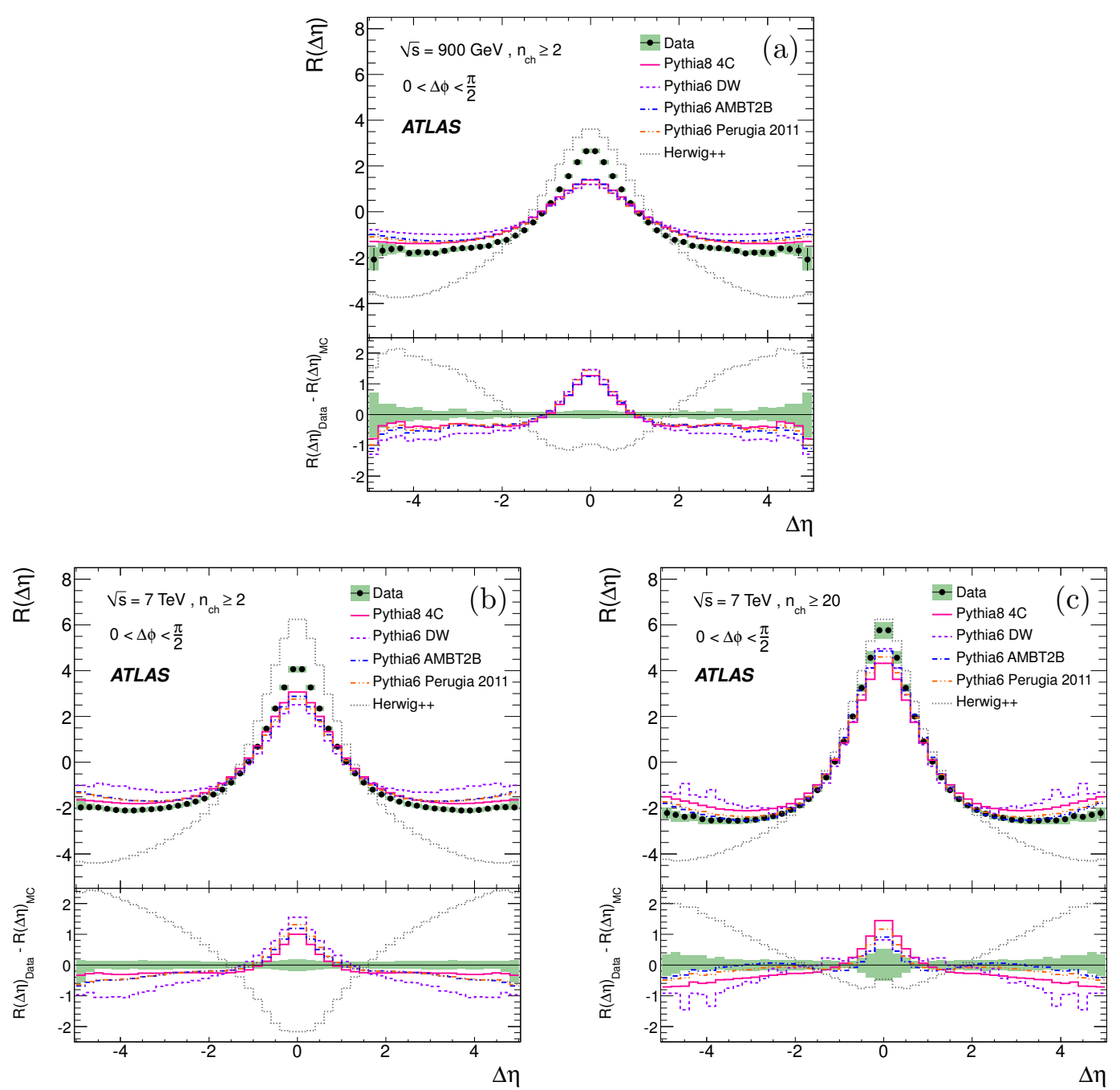

Figure 7. Corrected $R(\Delta \eta)$ two-particle correlation functions obtained by integrating the foreground and background distributions over $\Delta \phi$ between 0 and $\frac{\pi}{2}$ for data and the different Monte Carlo tunes at (a) $\sqrt{s}=900 \mathrm{GeV}$ and $7 \mathrm{TeV}$ for events with a charged-particle multiplicity (b) $n_{c h} \geq 2$ and (c) $n_{c h} \geq 20$.

Unlike the short-range correlation function, the $\Delta \phi$ dependence of the long-range correlation function does not exhibit a peak at $\Delta \phi=0$. This is shown in figure 10, which reveals a trough at $\Delta \phi=0$ while the away-side peak is still present. With the exception of PYTHIA 6 tune DW, the difference between the Monte Carlo models and the data is approximately flat in $\Delta \phi$. While the models here describe the shape of the correlation function, they do not describe the strength of the correlations. AMBT2B, Perugia 2011 and 4C are the best of the models considered here, although $4 \mathrm{C}$ diverges slightly more than the other two tunes when compared to the $n_{c h} \geq 20$ sample of events. This $\Delta \phi$ projection was recently studied in high-multiplicity events by the CMS Collaboration and a ridge-like structure was observed at $\Delta \phi=0$ in a specific kinematic range, different to the one studied here [28]. 

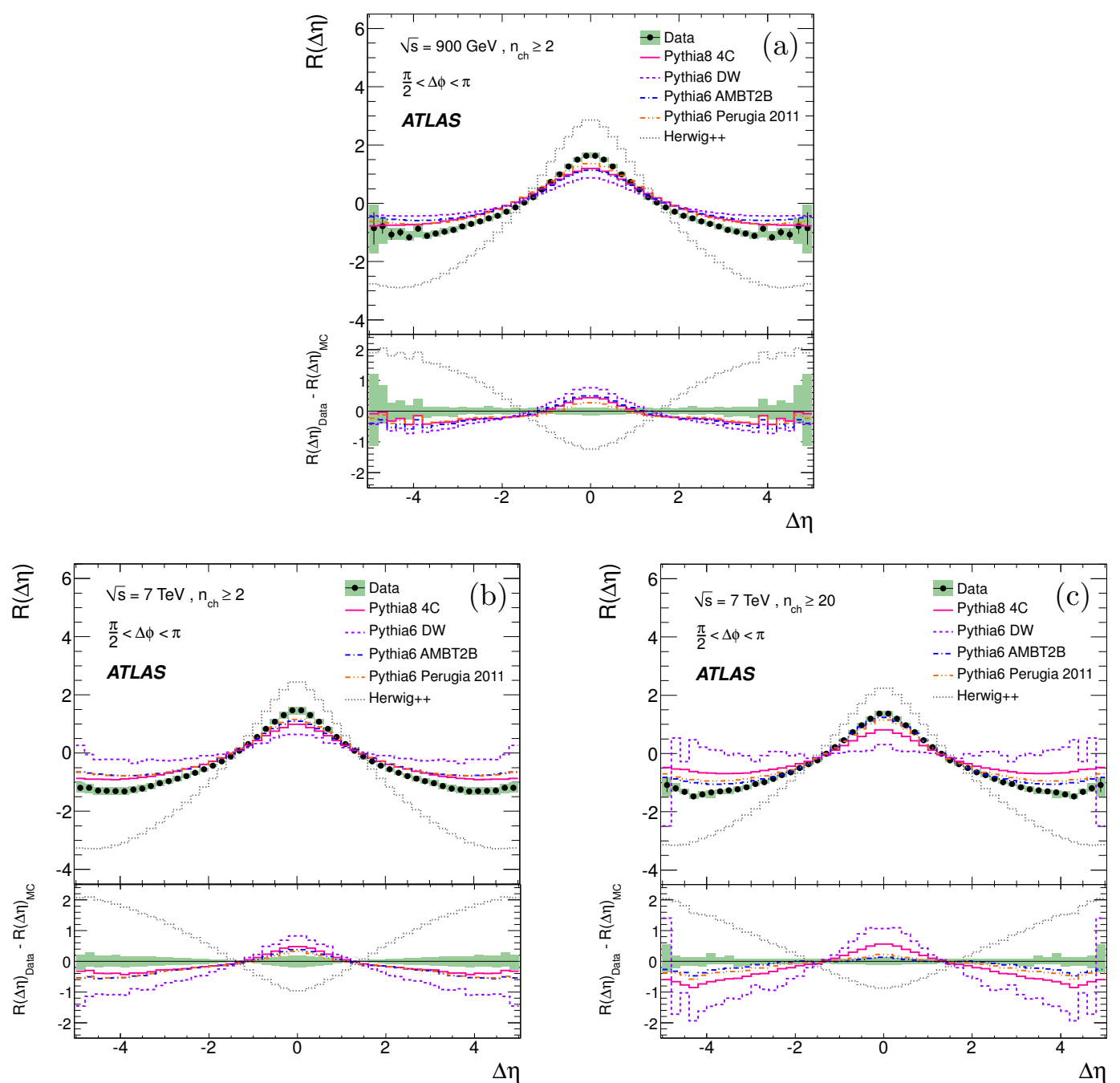

Figure 8. Corrected $R(\Delta \eta)$ two-particle correlation functions obtained by integrating the foreground and background distributions over $\Delta \phi$ between $\frac{\pi}{2}$ and $\pi$ for data and the different Monte Carlo tunes at (a) $\sqrt{s}=900 \mathrm{GeV}$ and $7 \mathrm{TeV}$ for events with a charged-particle multiplicity (b) $n_{c h} \geq 2$ and (c) $n_{c h} \geq 20$.

CMS has also studied the $p_{\mathrm{T}}$-inclusive two-particle angular correlation function at $\sqrt{s}=900 \mathrm{GeV}$ and $7 \mathrm{TeV}$. Their results [28] include the two-dimensional distributions, $R(\Delta \eta, \Delta \phi)$, and a single $R(\Delta \eta)$ projection where the correlation function has been integrated over $\Delta \phi$ between 0 and $\pi$. The phase-space used in their analysis is $p_{\mathrm{T}}>100 \mathrm{MeV}$ and $|\eta|<2.4$, a difference of 0.1 units in pseudorapidity with respect to the analysis presented in this paper. In comparing the $\Delta \eta$ dependence of the correlation function obtained by CMS with the results shown here, one should note a small and subtle difference in the definitions of the observables used in the two analyses. Whereas the analysis presented here measures the foreground, background and final correlation function inclusively for all event multiplicities within the event selection, the CMS analysis first determines the correlation 

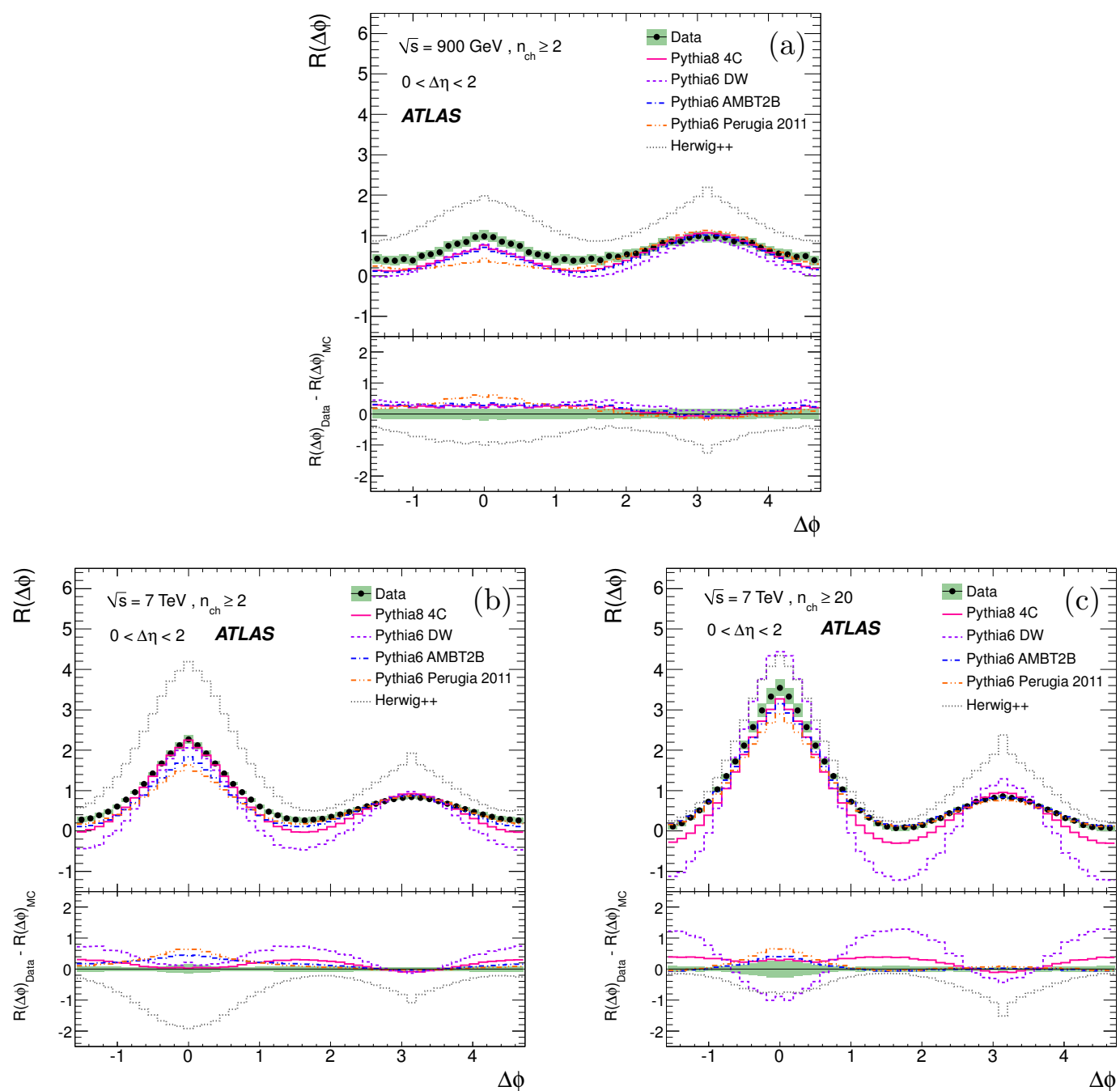

Figure 9. Corrected $R(\Delta \phi)$ two-particle correlation functions obtained by integrating $\Delta \eta$ between 0 and 2 for data and the different Monte Carlo tunes at (a) $\sqrt{s}=900 \mathrm{GeV}$ and $7 \mathrm{TeV}$ for events with a charged-particle multiplicity (b) $n_{c h} \geq 2$ and (c) $n_{c h} \geq 20$.

function in separate bins of multiplicity. The weighted average of the function in each bin is then computed to give an inclusive set of distributions. In order to see good agreement between these two observable definitions, one would need to correct the correlation function in each multiplicity bin when using the CMS definition and correct the multiplicity selection requirements used to define the multiplicity-dependent correlation function. The analysis performed by CMS was reproduced using the data presented here, and the results obtained were found to be in good agreement with those published by the CMS Collaboration. 

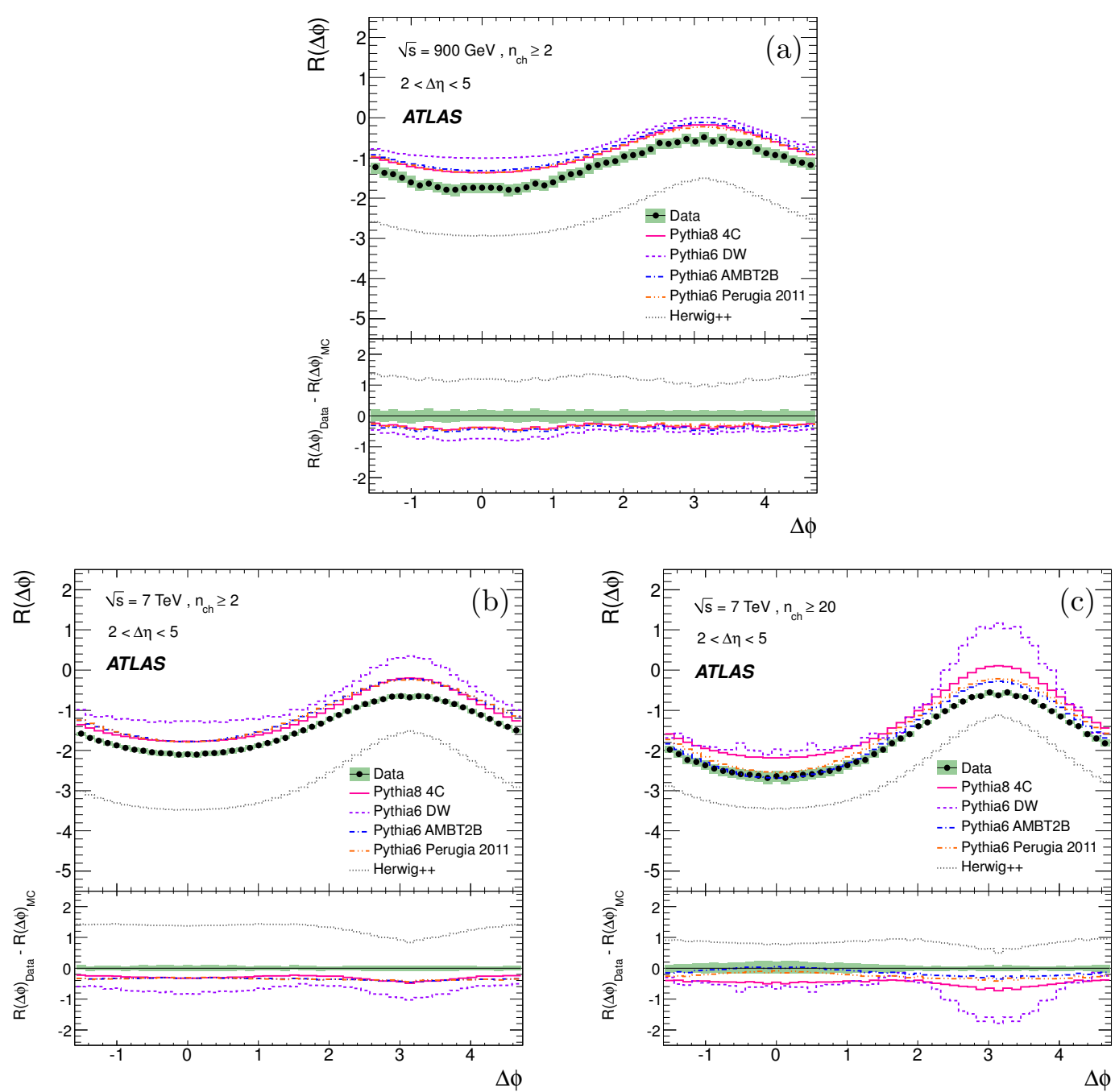

Figure 10. Corrected $R(\Delta \phi)$ two-particle correlation functions obtained by integrating the foreground and background distributions over $\Delta \eta$ between 2 and 5 for data and the different Monte Carlo tunes at (a) $\sqrt{s}=900 \mathrm{GeV}$ and $7 \mathrm{TeV}$ for events with a charged-particle multiplicity (b) $n_{c h} \geq 2$ and (c) $n_{c h} \geq 20$.

\section{$9 \quad$ Summary and conclusions}

The two-particle angular correlation functions in $\Delta \eta$ and $\Delta \phi$ have been measured using charged particles with $p_{\mathrm{T}}>100 \mathrm{MeV}$ and $|\eta|<2.5$. Events were recorded by the ATLAS detector at the LHC from proton-proton collisions at $\sqrt{s}=900 \mathrm{GeV}$ and $7 \mathrm{TeV}$, using a minimum bias trigger. In total, integrated luminosities of approximately $7 \mu \mathrm{b}^{-1}$ and $190 \mu \mathrm{b}^{-1}$ have been analysed, respectively. At $\sqrt{s}=7 \mathrm{TeV}$, two different phase-space regions defined by the charged particle multiplicity were investigated: $n_{c h} \geq 2$ and $n_{c h} \geq 20$. The structure of the correlation was explored in more detail by projecting the two-dimensional distributions into both $\Delta \eta$ and $\Delta \phi$. The results have been compared to Monte Carlo pre- 
dictions, which show a similar complex structure but fail to reproduce the strength of the correlations seen in the data. Overall, the AMBT2B tune of PYTHIA 6 and the 4C tune of PYTHIA 8 are the models that provide the best description, while HERWIG++ is the most discrepant in all of the distributions.

While many of the models shown here reproduce the general features of the two-particle correlation function, none of them provide a good quantitative description of the strength of the correlations. In order to properly describe the correlations, the phenomenology of soft particle production needs further improvement. Such improvement will, at a minimum, require the adjustment of existing models for diffraction and hadronisation. However, it may be that the changes required to describe these data will extend beyond the re-tuning of existing models.

\section{Acknowledgments}

We thank CERN for the very successful operation of the LHC, as well as the support staff from our institutions without whom ATLAS could not be operated efficiently.

We acknowledge the support of ANPCyT, Argentina; YerPhI, Armenia; ARC, Australia; BMWF, Austria; ANAS, Azerbaijan; SSTC, Belarus; CNPq and FAPESP, Brazil; NSERC, NRC and CFI, Canada; CERN; CONICYT, Chile; CAS, MOST and NSFC, China; COLCIENCIAS, Colombia; MSMT CR, MPO CR and VSC CR, Czech Republic; DNRF, DNSRC and Lundbeck Foundation, Denmark; EPLANET and ERC, European Union; IN2P3-CNRS, CEA-DSM/IRFU, France; GNAS, Georgia; BMBF, DFG, HGF, MPG and AvH Foundation, Germany; GSRT, Greece; ISF, MINERVA, GIF, DIP and Benoziyo Center, Israel; INFN, Italy; MEXT and JSPS, Japan; CNRST, Morocco; FOM and NWO, Netherlands; RCN, Norway; MNiSW, Poland; GRICES and FCT, Portugal; MERYS (MECTS), Romania; MES of Russia and ROSATOM, Russian Federation; JINR; MSTD, Serbia; MSSR, Slovakia; ARRS and MVZT, Slovenia; DST/NRF, South Africa; MICINN, Spain; SRC and Wallenberg Foundation, Sweden; SER, SNSF and Cantons of Bern and Geneva, Switzerland; NSC, Taiwan; TAEK, Turkey; STFC, the Royal Society and Leverhulme Trust, United Kingdom; DOE and NSF, United States of America.

The crucial computing support from all WLCG partners is acknowledged gratefully, in particular from CERN and the ATLAS Tier-1 facilities at TRIUMF (Canada), NDGF (Denmark, Norway, Sweden), CC-IN2P3 (France), KIT/GridKA (Germany), INFN-CNAF (Italy), NL-T1 (Netherlands), PIC (Spain), ASGC (Taiwan), RAL (U.K.) and BNL (U.S.A.) and in the Tier-2 facilities worldwide.

Open Access. This article is distributed under the terms of the Creative Commons Attribution License which permits any use, distribution and reproduction in any medium, provided the original author(s) and source are credited.

\section{References}

[1] B. Andersson, G. Gustafson and B. Nilsson-Almqvist, A model for low $p_{T}$ hadronic reactions, with generalizations to hadron-nucleus and nucleus-nucleus collisions, Nucl. Phys. B 281 (1987) 289 [INSPIRE]. 
[2] A. Morel and G. Plaut, How do clusters look in semi-inclusive cross-sections?, Nucl. Phys. B 78 (1974) 541 [INSPIRE].

[3] K. Eggert et al., Angular correlations between the charged particles produced in pp collisions at ISR energies, Nucl. Phys. B 86 (1975) 201 [INSPIRE].

[4] UA5 collaboration, R. Ansorge et al., Charged particle correlations in p̄p collisions at c.m. energies of $200 \mathrm{GeV}, 546 \mathrm{GeV}$ and $900 \mathrm{GeV}, \mathrm{Z}$. Phys. C 37 (1988) 191 [INSPIRE].

[5] PHOBOS collaboration, B. Alver et al., Cluster properties from two-particle angular correlations in $p+p$ collisions at $\sqrt{s}=200 \mathrm{GeV}$ and $410 \mathrm{GeV}$, Phys. Rev. C 75 (2007) 054913 [arXiv: 0704.0966] [INSPIRE].

[6] ATLAS collaboration, G. Aad et al., The ATLAS experiment at the CERN Large Hadron Collider, 2008 JINST 3 S08003 [INSPIRE].

[7] L. Evans and P. Bryant eds., LHC machine, 2008 JINST 3 S08001 [INSPIRE].

[8] T. Sjöstrand, S. Mrenna and P.Z. Skands, PYTHIA 6.4 physics and manual, JHEP 05 (2006) 026 [hep-ph/0603175] [INSPIRE].

[9] T. Sjöstrand, S. Mrenna and P.Z. Skands, A brief introduction to PYTHIA 8.1, Comput. Phys. Commun. 178 (2008) 852 [arXiv:0710.3820] [INSPIRE].

[10] M. Bähr et al., HERWIG++ physics and manual, Eur. Phys. J. C 58 (2008) 639 [arXiv: 0803.0883] [INSPIRE].

[11] M. Bähr, J.M. Butterworth, S. Gieseke and M.H. Seymour, Soft interactions in $H E R W I G++$, [arXiv:0905.4671] [INSPIRE].

[12] ATLAS collaboration, ATLAS tunes of PYTHIA 6 and PYTHIA 8 for MC11, ATL-PHYS-PUB-2011-009, CERN, Geneva Switzerland (2011).

[13] P.Z. Skands, Tuning Monte Carlo generators: the Perugia tunes, Phys. Rev. D 82 (2010) 074018 [arXiv: 1005. 3457] [INSPIRE].

[14] TeV4LHC QCD Working Group collaboration, M.G. Albrow et al., Tevatron-for-LHC report of the QCD working group, [hep-ph/0610012] [INSPIRE].

[15] R. Corke and T. Sjöstrand, Interleaved parton showers and tuning prospects, JHEP 03 (2011) 032 [arXiv:1011.1759] [INSPIRE].

[16] Energy-dependent UE tune webpage, http://projects.hepforge.org/herwig/trac/wiki/MB_UE_tunes.

[17] S. Gieseke et al., HERWIG++ 2.5 release note, [arXiv:1102.1672] [INSPIRE].

[18] J. Pumplin et al., New generation of parton distributions with uncertainties from global QCD analysis, JHEP 07 (2002) 012 [hep-ph/0201195] [INSPIRE].

[19] A. Sherstnev and R. Thorne, Parton distributions for LO generators, Eur. Phys. J. C 55 (2008) 553 [arXiv:0711.2473] [INSPIRE].

[20] P.Z. Skands, The Perugia tunes, [arXiv:0905.3418] [INSPIRE].

[21] ATLAS collaboration, ATLAS Monte Carlo tunes for MC09, ATL-PHYS-PUB-2010-002, CERN, Geneva Switzerland (2010).

[22] R. Engel, Photoproduction within the two component dual parton model. 1. Amplitudes and cross-sections, Z. Phys. C 66 (1995) 203 [InSPIRE]. 
[23] GEANT4 collaboration, S. Agostinelli et al., GEANT4: a simulation toolkit, Nucl. Instrum. Meth. A 506 (2003) 250 [INSPIRE].

[24] ATLAS collaboration, G. Aad et al., The ATLAS simulation infrastructure, Eur. Phys. J. C 70 (2010) 823 [arXiv: 1005.4568] [INSPIRE].

[25] ATLAS collaboration, G. Aad et al., Charged-particle multiplicities in pp interactions measured with the ATLAS detector at the LHC, New J. Phys. 13 (2011) 053033 [arXiv: 1012.5104] [INSPIRE].

[26] J.W. Monk and C. Oropeza-Barrera, The HBOM method for unfolding detector effects, submitted to Nucl. Instrum. Meth. (2011) [arXiv:1111.4896] [INSPIRE].

[27] ATLAS collaboration, G. Aad et al., Measurement of underlying event characteristics using charged particles in pp collisions at $\sqrt{s}=900 \mathrm{GeV}$ and $7 \mathrm{TeV}$ with the ATLAS detector, Phys. Rev. D 83 (2011) 112001 [arXiv:1012.0791] [INSPIRE].

[28] CMS collaboration, V. Khachatryan et al., Observation of long-range near-side angular correlations in proton-proton collisions at the LHC, JHEP 09 (2010) 091 [arXiv: 1009.4122] [INSPIRE]. 


\section{The ATLAS collaboration}

G. Aad $^{48}$, B. Abbott ${ }^{111}$, J. Abdallah ${ }^{11}$, A.A. Abdelalim ${ }^{49}$, A. Abdesselam ${ }^{118}$, O. Abdinov ${ }^{10}$, B. Abi ${ }^{112}$, M. Abolins ${ }^{88}$, O.S. AbouZeid ${ }^{158}$, H. Abramowicz ${ }^{153}$, H. Abreu $^{115}$, E. Acerbi ${ }^{89 a, 89 b}$, B.S. Acharya ${ }^{164 a, 164 b}$, L. Adamczyk ${ }^{37}$, D.L. Adams ${ }^{24}$, T.N. Addy ${ }^{56}$, J. Adelman ${ }^{176}$, M. Aderholz ${ }^{99}$, S. Adomeit ${ }^{98}$, P. Adragna ${ }^{75}$, T. Adye ${ }^{129}$, S. Aefsky ${ }^{22}$, J.A. Aguilar-Saavedra ${ }^{124 b, a}$, M. Aharrouche ${ }^{81}$, S.P. Ahlen ${ }^{21}$, F. Ahles ${ }^{48}$, A. Ahmad ${ }^{148}$, M. $\operatorname{Ahsan}^{40}$, G. Aielli133a,133b, T. Akdogan ${ }^{18 a}$, T.P.A. Åkesson ${ }^{79}$, G. Akimoto ${ }^{155}$, A.V. Akimov ${ }^{94}$, A. Akiyama ${ }^{66}$, M.S. Alam ${ }^{1}$, M.A. Alam ${ }^{76}$, J. Albert ${ }^{169}$, S. Albrand ${ }^{55}$, M. Aleksa ${ }^{29}$, I.N. Aleksandrov ${ }^{64}$, F. Alessandria ${ }^{89 a}$, C. Alexa ${ }^{25 a}$, G. Alexander ${ }^{153}$, G. Alexandre ${ }^{49}$, T. Alexopoulos ${ }^{9}$, M. Alhroob ${ }^{20}$, M. Aliev $^{15}$, G. Alimonti ${ }^{89 a}$, J. Alison ${ }^{120}$, M. Aliyev ${ }^{10}$, B.M.M. Allbrooke ${ }^{17}$, P.P. Allport ${ }^{73}$, S.E. Allwood-Spiers ${ }^{53}$, J. Almond ${ }^{82}$, A. Aloisio ${ }^{102 a, 102 b}$, R. Alon ${ }^{172}$, A. Alonso ${ }^{79}$, B. Alvarez Gonzalez ${ }^{88}$, M.G. Alviggi ${ }^{102 a, 102 b}$, K. Amako 65 , P. Amaral ${ }^{29}$, C. Amelung ${ }^{22}$, V.V. Ammosov ${ }^{128}$, A. Amorim ${ }^{124 a, b}$, G. Amorós ${ }^{167}$, N. Amram ${ }^{153}$, C. Anastopoulos ${ }^{29}$, L.S. Ancu ${ }^{16}$, N. Andari ${ }^{115}$, T. Andeen ${ }^{34}$, C.F. Anders $^{20}$, G. Anders ${ }^{58 a}$, K.J. Anderson ${ }^{30}$, A. Andreazza ${ }^{89 a, 89 b}$, V. Andrei ${ }^{58 a}$, ML. Andrieux ${ }^{55}$, X.S. Anduaga ${ }^{70}$, A. Angerami ${ }^{34}$, F. Anghinolfi ${ }^{29}$, A. Anisenkov ${ }^{107}$, N. Anjos ${ }^{124 a}$, A. Annovi ${ }^{47}$, A. Antonaki ${ }^{8}$, M. Antonelli ${ }^{47}$, A. Antonov ${ }^{96}$, J. Antos ${ }^{144 b}$, F. Anulli ${ }^{132 a}$, S. Aoun ${ }^{83}$, L. Aperio Bella ${ }^{4}$, R. Apolle ${ }^{118, c}$, G. Arabidze ${ }^{88}$, I. Aracena ${ }^{143}$, Y. Arai ${ }^{65}$, A.T.H. Arce ${ }^{44}$, S. Arfaoui ${ }^{148}$, J-F. Arguin ${ }^{14}$, E. Arik ${ }^{18 a, *}$, M. Arik ${ }^{18 a}$, A.J. Armbruster ${ }^{87}$, O. Arnaez ${ }^{81}$, C. Arnault ${ }^{115}$, A. Artamonov ${ }^{95}$, G. Artoni ${ }^{132 a, 132 b}$, D. Arutinov ${ }^{20}$, S. Asai ${ }^{155}$, R. Asfandiyarov ${ }^{173}$, S. Ask ${ }^{27}$, B. Åsman ${ }^{146 a, 146 b}$, L. Asquith ${ }^{5}$, K. Assamagan ${ }^{24}$, A. Astbury ${ }^{169}$, A. Astvatsatourov ${ }^{52}$, B. Aubert ${ }^{4}$, E. Auge ${ }^{115}$, K. Augsten ${ }^{127}$, M. Aurousseau ${ }^{145 a}$, G. Avolio ${ }^{163}$, R. Avramidou ${ }^{9}$, D. Axen ${ }^{168}$, C. Ay $^{54}$, G. Azuelos ${ }^{93, d}$, Y. Azuma ${ }^{155}$, M.A. Baak ${ }^{29}$, G. Baccaglioni89a, C. Bacci ${ }^{134 a, 134 b}$, A.M. Bach ${ }^{14}$, H. Bachacou ${ }^{136}$, K. Bachas ${ }^{29}$, M. Backes ${ }^{49}$, M. Backhaus ${ }^{20}$, E. Badescu ${ }^{25 a}$, P. Bagnaia ${ }^{132 a, 132 b}$, S. Bahinipati ${ }^{2}$, Y. Bai ${ }^{32 a}$, D.C. Bailey ${ }^{158}$, T. Bain ${ }^{158}$, J.T. Baines ${ }^{129}$, O.K. Baker ${ }^{176}$, M.D. Baker ${ }^{24}$, S. Baker ${ }^{77}$, E. Banas ${ }^{38}$, P. Banerjee ${ }^{93}$, Sw. Banerjee ${ }^{173}$, D. Banfi ${ }^{29}$, A. Bangert ${ }^{150}$, V. Bansal ${ }^{169}$, H.S. Bansil ${ }^{17}$, L. Barak ${ }^{172}$, S.P. Baranov ${ }^{94}$, A. Barashkou ${ }^{64}$, A. Barbaro Galtieri ${ }^{14}$, T. Barber $^{48}$, E.L. Barberio ${ }^{86}$, D. Barberis ${ }^{50 a, 50 b}$, M. Barbero ${ }^{20}$, D.Y. Bardin ${ }^{64}$, T. Barillari ${ }^{99}$, M. Barisonzi ${ }^{175}$, T. Barklow ${ }^{143}$, N. Barlow ${ }^{27}$, B.M. Barnett ${ }^{129}$, R.M. Barnett ${ }^{14}$, A. Baroncelli134a, G. Barone ${ }^{49}$, A.J. Barr ${ }^{118}$, F. Barreiro ${ }^{80}$, J. Barreiro Guimarães da Costa ${ }^{57}$, P. Barrillon ${ }^{115}$, R. Bartoldus ${ }^{143}$, A.E. Barton ${ }^{71}$, V. Bartsch ${ }^{149}$, R.L. Bates ${ }^{53}$, L. Batkova ${ }^{144 a}$, J.R. Batley ${ }^{27}$, A. Battaglia ${ }^{16}$, M. Battistin ${ }^{29}$, F. Bauer ${ }^{136}$, H.S. Bawa ${ }^{143, e}$, S. Beale ${ }^{98}$, T. Beau ${ }^{78}$, P.H. Beauchemin ${ }^{161}$, R. Beccherle ${ }^{50 a}$, P. Bechtle ${ }^{20}$, H.P. Beck ${ }^{16}$, S. Becker ${ }^{98}$, M. Beckingham ${ }^{138}$, K.H. Becks ${ }^{175}$, A.J. Beddall ${ }^{18 c}$, A. Beddall ${ }^{18 c}$, S. Bedikian ${ }^{176}$, V.A. Bednyakov ${ }^{64}$, C.P. Bee ${ }^{83}$, M. Begel ${ }^{24}$, S. Behar Harpaz ${ }^{152}$, P.K. Behera ${ }^{62}$, M. Beimforde ${ }^{99}$, C. Belanger-Champagne ${ }^{85}$, P.J. Bell ${ }^{49}$, W.H. Bell ${ }^{49}$, G. Bella ${ }^{153}$, L. Bellagamba ${ }^{19 a}$, F. Bellina ${ }^{29}$, M. Bellomo ${ }^{29}$, A. Belloni ${ }^{57}$, O. Beloborodova ${ }^{107, f}$, K. Belotskiy ${ }^{96}$, O. Beltramello ${ }^{29}$, S. Ben Ami ${ }^{152}$, O. Benary ${ }^{153}$, D. Benchekroun ${ }^{135 a}$, C. Benchouk ${ }^{83}$, M. Bendel ${ }^{81}$, N. Benekos ${ }^{165}$, Y. Benhammou ${ }^{153}$, E. Benhar Noccioli ${ }^{49}$, J.A. Benitez Garcia ${ }^{159 b}$, D.P. Benjamin ${ }^{44}$, M. Benoit ${ }^{115}$, J.R. Bensinger ${ }^{22}$, K. Benslama ${ }^{130}$, 
S. Bentvelsen ${ }^{105}$, D. Berge ${ }^{29}$, E. Bergeaas Kuutmann ${ }^{41}$, N. Berger ${ }^{4}$, F. Berghaus ${ }^{169}$, E. Berglund ${ }^{105}$, J. Beringer ${ }^{14}$, P. Bernat ${ }^{77}$, R. Bernhard ${ }^{48}$, C. Bernius ${ }^{24}$, T. Berry ${ }^{76}$, C. Bertella ${ }^{83}$, A. Bertin ${ }^{19 a, 19 b}$, F. Bertinelli ${ }^{29}$, F. Bertolucci ${ }^{122 a, 122 b}$, M.I. Besana ${ }^{89 a, 89 b}$, N. Besson ${ }^{136}$, S. Bethke ${ }^{99}$, W. Bhimji ${ }^{45}$, R.M. Bianchi ${ }^{29}$, M. Bianco ${ }^{72 a, 72 b}$, O. Biebel ${ }^{98}$, S.P. Bieniek ${ }^{77}$, K. Bierwagen ${ }^{54}$, J. Biesiada ${ }^{14}$, M. Biglietti134a, H. Bilokon ${ }^{47}$, M. Bindi ${ }^{19 a}, 19 b$, S. Binet ${ }^{115}$, A. Bingul ${ }^{18 c}$, C. Bini132a,132b , C. Biscarat ${ }^{178}$, U. Bitenc ${ }^{48}$, K.M. Black ${ }^{21}$, R.E. Blair ${ }^{5}$, J.-B. Blanchard ${ }^{136}$, G. Blanchot ${ }^{29}$, T. Blazek ${ }^{144 a}$, C. Blocker $^{22}$, J. Blocki ${ }^{38}$, A. Blondel ${ }^{49}$, W. Blum ${ }^{81}$, U. Blumenschein ${ }^{54}$, G.J. Bobbink ${ }^{105}$, V.B. Bobrovnikov ${ }^{107}$, S.S. Bocchetta ${ }^{79}$, A. Bocci ${ }^{44}$, C.R. Boddy ${ }^{118}$, M. Boehler ${ }^{41}$, J. Boek ${ }^{175}$, N. Boelaert ${ }^{35}$, J.A. Bogaerts ${ }^{29}$, A. Bogdanchikov ${ }^{107}$, A. Bogouch ${ }^{90, *}$, C. Bohm ${ }^{146 a}$, V. Boisvert ${ }^{76}$, T. Bold ${ }^{37}$, V. Boldea ${ }^{25 a}$, N.M. Bolnet ${ }^{136}$, M. Bona $^{75}$, V.G. Bondarenko ${ }^{96}$, M. Bondioli ${ }^{163}$, M. Boonekamp ${ }^{136}$, C.N. Booth ${ }^{139}$, S. Bordoni ${ }^{78}$, C. Borer ${ }^{16}$, A. Borisov ${ }^{128}$, G. Borissov ${ }^{71}$, I. Borjanovic ${ }^{12 a}$, M. Borri ${ }^{82}$, S. Borroni ${ }^{87}$, V. Bortolotto ${ }^{134 a, 134 b}$, K. Bos ${ }^{105}$, D. Boscherini ${ }^{19 a}$, M. Bosman ${ }^{11}$, H. Boterenbrood ${ }^{105}$, D. Botterill ${ }^{129}$, J. Bouchami ${ }^{93}$, J. Boudreau ${ }^{123}$, E.V. Bouhova-Thacker ${ }^{71}$, D. Boumediene ${ }^{33}$, C. Bourdarios ${ }^{115}$, N. Bousson ${ }^{83}$, A. Boveia ${ }^{30}$, J. Boyd ${ }^{29}$, I.R. Boyko ${ }^{64}$, N.I. Bozhko ${ }^{128}$, I. Bozovic-Jelisavcic ${ }^{12 b}$, J. Bracinik ${ }^{17}$, A. Braem ${ }^{29}$, P. Branchinii ${ }^{134 a}$, G.W. Brandenburg ${ }^{57}$, A. Brandt ${ }^{7}$, G. Brandt ${ }^{118}$, O. Brandt ${ }^{54}$, U. Bratzler ${ }^{156}$, B. Brau ${ }^{84}$, J.E. Brau ${ }^{114}$, H.M. Braun ${ }^{175}$, B. Brelier ${ }^{158}$, J. Bremer ${ }^{29}$, R. Brenner ${ }^{166}$, S. Bressler ${ }^{172}$, D. Breton ${ }^{115}$, D. Britton ${ }^{53}$, F.M. Brochu ${ }^{27}$, I. Brock ${ }^{20}$, R. Brock ${ }^{88}$, T.J. Brodbeck ${ }^{71}$, E. Brodet ${ }^{153}$, F. Broggi ${ }^{89 a}$, C. Bromberg ${ }^{88}$, J. Bronner ${ }^{99}$, G. Brooijmans ${ }^{34}$, W.K. Brooks ${ }^{31 b}$, G. Brown ${ }^{82}$, H. Brown ${ }^{7}$, P.A. Bruckman de Renstrom ${ }^{38}$, D. Bruncko ${ }^{144 b}$, R. Bruneliere ${ }^{48}$, S. Brunet ${ }^{60}$, A. Bruni ${ }^{19 a}$, G. Bruni ${ }^{19 a}$, M. Bruschi ${ }^{19 a}$, T. Buanes ${ }^{13}$, Q. Buat ${ }^{55}$, F. Bucci ${ }^{49}$, J. Buchanan ${ }^{118}$, N.J. Buchanan ${ }^{2}$, P. Buchholz ${ }^{141}$, R.M. Buckingham ${ }^{118}$, A.G. Buckley ${ }^{45}$, S.I. Buda ${ }^{25 a}$, I.A. Budagov ${ }^{64}$, B. Budick ${ }^{108}$, V. Büscher ${ }^{81}$, L. Bugge ${ }^{117}$, O. Bulekov ${ }^{96}$, M. Bunse ${ }^{42}$, T. Buran ${ }^{117}$, H. Burckhart ${ }^{29}$, S. Burdin ${ }^{73}$, T. Burgess ${ }^{13}$, S. Burke ${ }^{129}$, E. Busato ${ }^{33}$, P. Bussey ${ }^{53}$, C.P. Buszello ${ }^{166}$, F. Butin ${ }^{29}$, B. Butler ${ }^{143}$, J.M. Butler ${ }^{21}$, C.M. Buttar ${ }^{53}$, J.M. Butterworth ${ }^{77}$, W. Buttinger ${ }^{27}$, S. Cabrera Urbán $^{167}$, D. Caforio ${ }^{19 a, 19 b}$, O. Cakir ${ }^{3 a}$, P. Calafiura ${ }^{14}$, G. Calderini ${ }^{78}$, P. Calfayan ${ }^{98}$, R. Calkins ${ }^{106}$, L.P. Caloba ${ }^{23 a}$, R. Caloi ${ }^{132 a, 132 b}$, D. Calvet ${ }^{33}$, S. Calvet ${ }^{33}$, R. Camacho Toro ${ }^{33}$, P. Camarri133a,133b , M. Cambiaghi119a,119b ${ }^{13}$ D. Cameron ${ }^{117}$, L.M. Caminada ${ }^{14}$, S. Campana ${ }^{29}$, M. Campanelli ${ }^{77}$, V. Canale ${ }^{102 a, 102 b}$, F. Canelli ${ }^{30, g}$, A. Canepa ${ }^{159 a}$, J. Cantero ${ }^{80}$, L. Capasso ${ }^{102 a, 102 b}$, M.D.M. Capeans Garrido ${ }^{29}$ I. Caprini2 $^{25 a}$, M. Caprini ${ }^{25 a}$, D. Capriotti ${ }^{99}$, M. Capua ${ }^{36 a, 36 b}$, R. Caputo $^{81}$, C. Caramarcu ${ }^{24}$, R. Cardarelli ${ }^{133 a}$, T. Carli $^{29}$, G. Carlino ${ }^{102 a}$, L. Carminati ${ }^{89 a, 89 b}$, B. Caron $^{85}$, S. Caron ${ }^{104}$, G.D. Carrillo Montoya ${ }^{173}$, A.A. Carter ${ }^{75}$, J.R. Carter ${ }^{27}$, J. Carvalho ${ }^{124 a, h}$, D. Casadei ${ }^{108}$, M.P. Casado ${ }^{11}$, M. Cascella ${ }^{122 a, 122 b}$, C. Caso $^{50 a, 50 b, *}$, A.M. Castaneda Hernandez ${ }^{173}$, E. Castaneda-Miranda ${ }^{173}$, V. Castillo Gimenez ${ }^{167}$, N.F. Castro ${ }^{124 a}$, G. Cataldi ${ }^{72 a}$, F. Cataneo $^{29}$, A. Catinaccio $^{29}$, J.R. Catmore ${ }^{29}$, A. Cattai29, G. Cattani ${ }^{133 a, 133 b}$, S. Caughron ${ }^{88}$, D. Cauz $^{164 a, 164 c}$, P. Cavalleri ${ }^{78}$, D. Cavalli ${ }^{89 a}$, M. Cavalli-Sforza ${ }^{11}$, V. Cavasinni ${ }^{122 a, 122 b}$, F. Ceradini ${ }^{134 a, 134 b}$, A.S. Cerqueira ${ }^{23 b}$, A. Cerri ${ }^{29}$, L. Cerrito ${ }^{75}$, F. Cerutti $^{47}$, S.A. Cetin ${ }^{18 b}$, F. Cevenini ${ }^{102 a, 102 b}$, A. Chafaq ${ }^{135 a}$, D. Chakraborty ${ }^{106}$, K. Chan $^{2}$, B. Chapleau ${ }^{85}$, J.D. Chapman ${ }^{27}$, J.W. Chapman ${ }^{87}$, E. Chareyre ${ }^{78}$, 
D.G. Charlton ${ }^{17}$, V. Chavda ${ }^{82}$, C.A. Chavez Barajas $^{29}$, S. Cheatham ${ }^{85}$, S. Chekanov ${ }^{5}$, S.V. Chekulaev ${ }^{159 a}$, G.A. Chelkov ${ }^{64}$, M.A. Chelstowska ${ }^{104}$, C. Chen ${ }^{63}$, H. Chen $^{24}$, S. Chen ${ }^{32 c}$, T. Chen ${ }^{32 c}$, X. Chen ${ }^{173}$, S. Cheng ${ }^{32 a}$, A. Cheplakov ${ }^{64}$, V.F. Chepurnov ${ }^{64}$, R. Cherkaoui El Moursli ${ }^{135 e}$, V. Chernyatin ${ }^{24}$, E. Cheu ${ }^{6}$, S.L. Cheung ${ }^{158}$, L. Chevalier ${ }^{136}$, G. Chiefari ${ }^{102 a, 102 b}$, L. Chikovani ${ }^{51 a}$, J.T. Childers ${ }^{29}$, A. Chilingarov ${ }^{71}$, G. Chiodini $^{72 a}$, A.S. Chisholm ${ }^{17}$, M.V. Chizhov $^{64}$, G. Choudalakis $^{30}$, S. Chouridou ${ }^{137}$, I.A. Christidi ${ }^{77}$, A. Christov ${ }^{48}$, D. Chromek-Burckhart ${ }^{29}$, M.L. Chu ${ }^{151}$, J. Chudoba ${ }^{125}$, G. Ciapetti ${ }^{132 a, 132 b}$, K. $\mathrm{Ciba}^{37}$, A.K. Ciftci ${ }^{3 a}$, R. Ciftci ${ }^{3 a}$, D. Cinca ${ }^{33}$, V. Cindro ${ }^{74}$, M.D. Ciobotaru ${ }^{163}$,

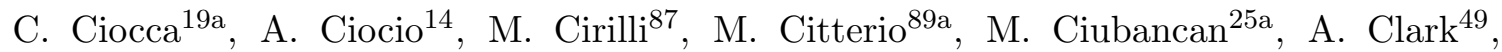
P.J. Clark ${ }^{45}$, W. Cleland ${ }^{123}$, J.C. Clemens ${ }^{83}$, B. Clement ${ }^{55}$, C. Clement ${ }^{146 a, 146 b}$, R.W. Clifft ${ }^{129}$, Y. Coadou ${ }^{83}$, M. Cobal ${ }^{164 a, 164 c}$, A. Coccaro ${ }^{173}$, J. Cochran ${ }^{63}$, P. Coe ${ }^{118}$, J.G. Cogan ${ }^{143}$, J. Coggeshall ${ }^{165}$, E. Cogneras ${ }^{178}$, J. Colas ${ }^{4}$, A.P. Colijn ${ }^{105}$, N.J. Collins ${ }^{17}$, C. Collins-Tooth ${ }^{53}$, J. Collot ${ }^{55}$, G. Colon ${ }^{84}$, P. Conde Muiño ${ }^{124 a}$, E. Coniavitis ${ }^{118}$, M.C. Conidi ${ }^{11}$, M. Consonni ${ }^{104}$, V. Consorti ${ }^{48}$, S. Constantinescu ${ }^{25 a}$, C. Conta ${ }^{119 a, 119 b}$, F. Conventi ${ }^{102 a, i}$, J. Cook ${ }^{29}$, M. Cooke ${ }^{14}$, B.D. Cooper ${ }^{77}$, A.M. CooperSarkar $^{118}$, K. Copic $^{14}$, T. Cornelissen ${ }^{175}$, M. Corradi ${ }^{19 a}$, F. Corriveau ${ }^{85, j}$, A. CortesGonzalez $^{165}$, G. Cortiana ${ }^{99}$, G. Costa ${ }^{89 a}$, M.J. Costa ${ }^{167}$, D. Costanzo ${ }^{139}$, T. Costin $^{30}$, D. Côté ${ }^{29}$, R. Coura Torres ${ }^{23 a}$, L. Courneyea ${ }^{169}$, G. Cowan ${ }^{76}$, C. Cowden ${ }^{27}$, B.E. $\operatorname{Cox}^{82}$, K. Cranmer ${ }^{108}$, F. Crescioli ${ }^{122 a, 122 b}$, M. Cristinziani ${ }^{20}$, G. Crosetti ${ }^{36 a, 36 b}$, R. Crupi ${ }^{72 a, 72 b}$, S. Crépé-Renaudin ${ }^{55}$, C.-M. Cuciuc ${ }^{25 a}$, C. Cuenca Almenar ${ }^{176}$, T. Cuhadar Donszelmann ${ }^{139}$, M. Curatolo ${ }^{47}$, C.J. Curtis ${ }^{17}$, C. Cuthbert ${ }^{150}$, P. Cwetanski ${ }^{60}$, H. Czirr ${ }^{141}$, P. Czodrowski ${ }^{43}$, Z. Czyczula ${ }^{176}$, S. D'Auria ${ }^{53}$, M. D'Onofrio ${ }^{73}$, A. D'Orazio ${ }^{132 a, 132 b}$, P.V.M. Da Silva ${ }^{23 a}$, C. Da Via ${ }^{82}$, W. Dabrowski ${ }^{37}$, T. Dai $^{87}$, C. Dallapiccola ${ }^{84}$, M. Dam ${ }^{35}$, M. Dameri ${ }^{50 a, 50 b}$, D.S. Damiani ${ }^{137}$, H.O. Danielsson ${ }^{29}$, D. Dannheim ${ }^{99}$, V. Dao ${ }^{49}$, G. Darbo ${ }^{50 a}$, G.L. Darlea ${ }^{25 b}$, W. Davey ${ }^{20}$, T. Davidek ${ }^{126}$, N. Davidson ${ }^{86}$, R. Davidson ${ }^{71}$, E. Davies ${ }^{118, c}$, M. Davies ${ }^{93}$, A.R. Davison ${ }^{77}$, Y. Davygora ${ }^{58 a}$, E. Dawe ${ }^{142}$, I. Dawson ${ }^{139}$, J.W. Dawson ${ }^{5, *}$, R.K. Daya-Ishmukhametova ${ }^{22}$, K. De ${ }^{7}$, R. de Asmundis ${ }^{102 a}$, S. De Castro ${ }^{19 a, 19 b}$, P.E. De Castro Faria Salgado ${ }^{24}$, S. De $\mathrm{Cecco}^{78}$, J. de Graat ${ }^{98}$, N. De Groot ${ }^{104}$, P. de Jong ${ }^{105}$, C. De La Taille ${ }^{115}$, H. De la Torre ${ }^{80}$, B. De Lotto ${ }^{164 a, 164 c}$, L. de Mora ${ }^{71}$, L. De Nooij ${ }^{105}$, D. De Pedis ${ }^{132 a}$, A. De Salvo ${ }^{132 a}$, U. De Sanctis ${ }^{164 a, 164 c}$, A. De Santo ${ }^{149}$, J.B. De Vivie De Regie ${ }^{115}$, S. Dean ${ }^{77}$, W.J. Dearnaley ${ }^{71}$, R. Debbe ${ }^{24}$, C. Debenedetti ${ }^{45}$, D.V. Dedovich ${ }^{64}$, J. Degenhardt ${ }^{120}$, M. Dehchar ${ }^{118}$, C. Del Papa ${ }^{164 a, 164 c}$, J. Del Peso ${ }^{80}$, T. Del Prete ${ }^{122 a, 122 b}$, T. Delemontex ${ }^{55}$, M. Deliyergiyev ${ }^{74}$, A. Dell'Acqua ${ }^{29}$, L. Dell'Asta ${ }^{21}$, M. Della Pietra ${ }^{102 a, ~} i$, D. della Volpe 102a,102b, M. Delmastro ${ }^{4}$, N. Delruelle ${ }^{29}$, P.A. Delsart ${ }^{55}$, C. Deluca ${ }^{148}$, S. Demers ${ }^{176}$, M. Demichev ${ }^{64}$, B. Demirkoz ${ }^{11, k}$, J. Deng ${ }^{163}$, S.P. Denisov ${ }^{128}$, D. Derendarz ${ }^{38}$, J.E. Derkaoui ${ }^{135 d}$, F. Derue ${ }^{78}$, P. Dervan ${ }^{73}$, K. Desch ${ }^{20}$, E. Devetak ${ }^{148}$, P.O. Deviveiros ${ }^{105}$, A. Dewhurst ${ }^{129}$, B. DeWilde ${ }^{148}$, S. Dhaliwal ${ }^{158}$, R. Dhullipudi ${ }^{24, l}$, A. Di Ciaccio 133a,133b, L. Di Ciaccio $^{4}$, A. Di Girolamo ${ }^{29}$, B. Di Girolamo ${ }^{29}$, S. Di Luise ${ }^{134 a, 134 b}$, A. Di Mattia ${ }^{173}$, B. Di Micco ${ }^{29}$, R. Di Nardo ${ }^{47}$, A. Di Simone ${ }^{133 a, 133 b}$,

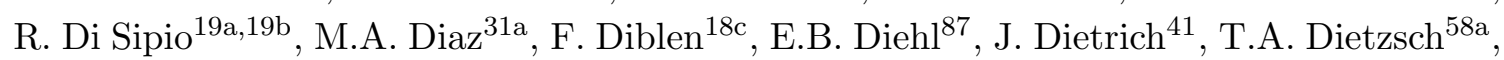
S. Diglio ${ }^{86}$, K. Dindar Yagci ${ }^{39}$, J. Dingfelder ${ }^{20}$, C. Dionisi ${ }^{132 a, 132 b}$, P. Dita $^{25 a}$, S. Dita $^{25 a}$, F. Dittus ${ }^{29}$, F. Djama ${ }^{83}$, T. Djobava ${ }^{51 b}$, M.A.B. do Vale ${ }^{23 c}$, A. Do Valle Wemans ${ }^{124 a}$, 
T.K.O. Doan ${ }^{4}$, M. Dobbs ${ }^{85}$, R. Dobinson ${ }^{29, *}$, D. Dobos ${ }^{29}$, E. Dobson ${ }^{29, m}$, J. Dodd ${ }^{34}$, C. Doglioni ${ }^{49}$, T. Doherty ${ }^{53}$, Y. Doi ${ }^{65, *}$, J. Dolejsi ${ }^{126}$, I. Dolenc ${ }^{74}$, Z. Dolezal ${ }^{126}$, B.A. Dolgoshein ${ }^{96, *}$, T. Dohmae ${ }^{155}$, M. Donadelli ${ }^{23 d}$, M. Donega ${ }^{120}$, J. Donini ${ }^{33}$, J. Dopke ${ }^{29}$, A. Doria ${ }^{102 a}$, A. Dos Anjos ${ }^{173}$, M. Dosil ${ }^{11}$, A. Dotti ${ }^{122 a, 122 b}$, M.T. Dova ${ }^{70}$, J.D. Dowell ${ }^{17}$, A.D. Doxiadis ${ }^{105}$, A.T. Doyle ${ }^{53}$, Z. Drasal ${ }^{126}$, J. Drees ${ }^{175}$, N. Dressnandt ${ }^{120}$, H. Drevermann ${ }^{29}$, C. Driouichi ${ }^{35}$, M. Dris ${ }^{9}$, J. Dubbert ${ }^{99}$, S. Dube ${ }^{14}$, E. Duchovni ${ }^{172}$, G. Duckeck ${ }^{98}$, A. Dudarev ${ }^{29}$, F. Dudziak ${ }^{63}$, M. Dührssen 29, I.P. Duerdoth ${ }^{82}$, L. Duflot ${ }^{115}$, M-A. Dufour ${ }^{85}$, M. Dunford ${ }^{29}$, H. Duran Yildiz ${ }^{3 a}$, R. Duxfield ${ }^{139}$, M. Dwuznik ${ }^{37}$, F. Dydak ${ }^{29}$, M. Düren ${ }^{52}$, W.L. Ebenstein ${ }^{44}$, J. Ebke ${ }^{98}$, S. Eckweiler ${ }^{81}$, K. Edmonds ${ }^{81}$, C.A. Edwards ${ }^{76}$, N.C. Edwards ${ }^{53}$, W. Ehrenfeld ${ }^{41}$, T. Ehrich ${ }^{99}$, T. Eifert ${ }^{143}$, G. Eigen ${ }^{13}$, K. Einsweiler ${ }^{14}$, E. Eisenhandler ${ }^{75}$, T. Ekelof ${ }^{166}$, M. El Kacimi ${ }^{135 c}$, M. Ellert ${ }^{166}$, S. Elles ${ }^{4}$, F. Ellinghaus ${ }^{81}$, K. Ellis ${ }^{75}$, N. Ellis ${ }^{29}$, J. Elmsheuser ${ }^{98}$, M. Elsing ${ }^{29}$, D. Emeliyanov ${ }^{129}$, R. Engelmann ${ }^{148}$, A. Engl ${ }^{98}$, B. Epp ${ }^{61}$, A. Eppig ${ }^{87}$, J. Erdmann ${ }^{54}$, A. Ereditato ${ }^{16}$, D. Eriksson ${ }^{146 a}$, J. Ernst ${ }^{1}$, M. Ernst ${ }^{24}$, J. Ernwein ${ }^{136}$, D. Errede ${ }^{165}$, S. Errede ${ }^{165}$, E. Ertel ${ }^{81}$, M. Escalier ${ }^{115}$, C. Escobar ${ }^{123}$, X. Espinal Curull ${ }^{11}$, B. Esposito ${ }^{47}$, F. Etienne ${ }^{83}$, A.I. Etienvre ${ }^{136}$, E. Etzion ${ }^{153}$, D. Evangelakou ${ }^{54}$, H. Evans $^{60}$, L. Fabbri ${ }^{19 a, 19 b}$, C. Fabre ${ }^{29}$, R.M. Fakhrutdinov ${ }^{128}$, S. Falciano ${ }^{132 a}$, Y. Fang ${ }^{173}$, M. Fanti ${ }^{89 a, 89 b}$, A. Farbin ${ }^{7}$, A. Farilla ${ }^{134 a}$, J. Farley ${ }^{148}$, T. Farooque ${ }^{158}$, S.M. Farrington ${ }^{118}$, P. Farthouat ${ }^{29}$, P. Fassnacht ${ }^{29}$, D. Fassouliotis ${ }^{8}$, B. Fatholahzadeh ${ }^{158}$, A. Favareto ${ }^{89 a, 89 b}$, L. Fayard ${ }^{115}$, S. Fazio ${ }^{36 a, 36 b}$, R. Febbraro ${ }^{33}$, P. Federic ${ }^{144 a}$, O.L. Fedin ${ }^{121}$, W. Fedorko ${ }^{88}$, M. Fehling-Kaschek ${ }^{48}$, L. Feligioni ${ }^{83}$, D. Fellmann ${ }^{5}$, C. Feng ${ }^{32 d}$, E.J. Feng ${ }^{30}$, A.B. Fenyuk ${ }^{128}$, J. Ferencei ${ }^{144 b}$, J. Ferland ${ }^{93}$, W. Fernando ${ }^{109}$, S. Ferrag ${ }^{53}$, J. Ferrando ${ }^{53}$, V. Ferrara ${ }^{41}$, A. Ferrari ${ }^{166}$, P. Ferrari ${ }^{105}$, R. Ferrari ${ }^{119 a}$, D.E. Ferreira de Lima $^{53}$, A. Ferrer ${ }^{167}$, M.L. Ferrer ${ }^{47}$, D. Ferrere ${ }^{49}$, C. Ferretti ${ }^{87}$, A. Ferretto Parodi ${ }^{50 a}, 50 \mathrm{~b}$, M. Fiascaris ${ }^{30}$, F. Fiedler ${ }^{81}$, A. Filipčič ${ }^{74}$, A. Filippas ${ }^{9}$, F. Filthaut ${ }^{104}$, M. Fincke-Keeler ${ }^{169}$, M.C.N. Fiolhais ${ }^{124 a}, h$, L. Fiorini ${ }^{167}$, A. Firan ${ }^{39}$, G. Fischer ${ }^{41}$, P. Fischer ${ }^{20}$, M.J. Fisher ${ }^{109}$, M. Flechl ${ }^{48}$, I. Fleck ${ }^{141}$, J. Fleckner ${ }^{81}$, P. Fleischmann ${ }^{174}$, S. Fleischmann ${ }^{175}$, T. Flick ${ }^{175}$, A. Floderus ${ }^{79}$, L.R. Flores Castillo ${ }^{173}$, M.J. Flowerdew ${ }^{99}$, M. Fokitis ${ }^{9}$, T. Fonseca Martin ${ }^{16}$, D.A. Forbush ${ }^{138}$, A. Formica ${ }^{136}$, A. Forti ${ }^{82}$, D. Fortin ${ }^{159 a}$, J.M. Foster ${ }^{82}$, D. Fournier ${ }^{115}$, A. Foussat ${ }^{29}$, A.J. Fowler ${ }^{44}$, K. Fowler ${ }^{137}$, H. Fox ${ }^{71}$, P. Francavilla ${ }^{11}$, S. Franchino ${ }^{119 a, 119 b}$, D. Francis ${ }^{29}$, T. Frank ${ }^{172}$, M. Franklin ${ }^{57}$, S. Franz $^{29}$, M. Fraternali ${ }^{119 a, 119 b}$, S. Fratina ${ }^{120}$, S.T. French ${ }^{27}$, F. Friedrich ${ }^{43}$, R. Froeschl ${ }^{29}$, D. Froidevaux ${ }^{29}$, J.A. Frost ${ }^{27}$, C. Fukunaga ${ }^{156}$, E. Fullana Torregrosa ${ }^{29}$, J. Fuster ${ }^{167}$, C. Gabaldon ${ }^{29}$, O. Gabizon ${ }^{172}$, T. Gadfort ${ }^{24}$, S. Gadomski ${ }^{49}$, G. Gagliardi ${ }^{50 a, 50 b}$, P. Gagnon ${ }^{60}$, C. Galea ${ }^{98}$, E.J. Gallas ${ }^{118}$, V. Gallo ${ }^{16}$, B.J. Gallop ${ }^{129}$, P. Gallus ${ }^{125}$, K.K. Gan ${ }^{109}$, Y.S. Gao ${ }^{143, e}$, V.A. Gapienko ${ }^{128}$, A. Gaponenko ${ }^{14}$, F. Garberson ${ }^{176}$, M. Garcia-Sciveres ${ }^{14}$, C. García ${ }^{167}$, J.E. García Navarro ${ }^{167}$, R.W. Gardner ${ }^{30}$, N. Garelli ${ }^{29}$, H. Garitaonandia ${ }^{105}$, V. Garonne ${ }^{29}$, J. Garvey ${ }^{17}$, C. Gatti $^{47}$, G. Gaudio ${ }^{119 a}$, B. Gaur ${ }^{141}$, L. Gauthier ${ }^{136}$, I.L. Gavrilenko ${ }^{94}$, C. Gay ${ }^{168}$, G. Gaycken ${ }^{20}$, J-C. Gayde ${ }^{29}$, E.N. Gazis ${ }^{9}$, P. $\mathrm{Ge}^{32 \mathrm{~d}}$, C.N.P. Gee ${ }^{129}$, D.A.A. Geerts ${ }^{105}$, Ch. Geich-Gimbel ${ }^{20}$, K. Gellerstedt ${ }^{146 a, 146 \mathrm{~b}}$, C. Gemme ${ }^{50 a}$, A. Gemmell ${ }^{53}$, M.H. Genest ${ }^{55}$, S. Gentile ${ }^{132 a, 132 b}$, M. George ${ }^{54}$, S. George ${ }^{76}$, P. Gerlach ${ }^{175}$, A. Gershon ${ }^{153}$, C. Geweniger ${ }^{58 a}$, H. Ghazlane ${ }^{135 b}$, N. Ghodbane ${ }^{33}$, B. Giacobbe ${ }^{19 a}$, S. Giagu ${ }^{132 a, 132 b}$, V. Giakoumopoulou ${ }^{8}$, V. Giangiobbe ${ }^{11}$, 
F. Gianotti ${ }^{29}$, B. Gibbard ${ }^{24}$, A. Gibson ${ }^{158}$, S.M. Gibson ${ }^{29}$, L.M. Gilbert ${ }^{118}$, V. Gilewsky ${ }^{91}$, D. Gillberg ${ }^{28}$, A.R. Gillman ${ }^{129}$, D.M. Gingrich ${ }^{2, d}$, J. Ginzburg ${ }^{153}$, N. Giokaris ${ }^{8}$, M.P. Giordani ${ }^{164 c}$, R. Giordano ${ }^{102 a, 102 b}$, F.M. Giorgi ${ }^{15}$, P. Giovannini ${ }^{99}$, P.F. Giraud ${ }^{136}$, D. Giugni ${ }^{89 a}$, M. Giunta ${ }^{93}$, P. Giusti ${ }^{19 a}$, B.K. Gjelsten ${ }^{117}$, L.K. Gladilin ${ }^{97}$, C. Glasman ${ }^{80}$, J. Glatzer ${ }^{48}$, A. Glazov ${ }^{41}$, K.W. Glitza ${ }^{175}$, G.L. Glonti ${ }^{64}$, J.R. Goddard ${ }^{75}$, J. Godfrey ${ }^{142}$, J. Godlewski ${ }^{29}$, M. Goebel ${ }^{41}$, T. Göpfert ${ }^{43}$, C. Goeringer ${ }^{81}$, C. Gössling ${ }^{42}$, T. Göttfert ${ }^{99}$, S. Goldfarb ${ }^{87}$, T. Golling ${ }^{176}$, A. Gomes ${ }^{124 a, b}$, L.S. Gomez Fajardo ${ }^{41}$, R. Gonçalo ${ }^{76}$, J. Goncalves Pinto Firmino Da Costa ${ }^{41}$, L. Gonella ${ }^{20}$, A. Gonidec ${ }^{29}$, S. Gonzalez ${ }^{173}$, S. González de la Hoz ${ }^{167}$, G. Gonzalez Parra ${ }^{11}$, M.L. Gonzalez Silva ${ }^{26}$, S. GonzalezSevilla $^{49}$, J.J. Goodson ${ }^{148}$, L. Goossens ${ }^{29}$, P.A. Gorbounov ${ }^{95}$, H.A. Gordon ${ }^{24}$, I. Gorelov ${ }^{103}$, G. Gorfine ${ }^{175}$, B. Gorini ${ }^{29}$, E. Gorini ${ }^{72 a, 72 b}$, A. Gorišek ${ }^{74}$, E. Gornicki ${ }^{38}$, S.A. Gorokhov ${ }^{128}$, V.N. Goryachev ${ }^{128}$, B. Gosdzik ${ }^{41}$, M. Gosselink ${ }^{105}$, M.I. Gostkin ${ }^{64}$, I. Gough Eschrich ${ }^{163}$, M. Gouighri ${ }^{135 a}$, D. Goujdami ${ }^{135 c}$, M.P. Goulette ${ }^{49}$, A.G. Goussiou ${ }^{138}$, C. Goy ${ }^{4}$, S. Gozpinar ${ }^{22}$, I. Grabowska-Bold ${ }^{37}$, P. Grafström ${ }^{29}$, K-J. Grahn ${ }^{41}$, F. Grancagnolo ${ }^{72 a}$, S. Grancagnolo ${ }^{15}$, V. Grassi ${ }^{148}$, V. Gratchev ${ }^{121}$, N. Grau ${ }^{34}$, H.M. Gray ${ }^{29}$, J.A. Gray ${ }^{148}$, E. Graziani ${ }^{134 a}$, O.G. Grebenyuk ${ }^{121}$, T. Greenshaw ${ }^{73}$, Z.D. Greenwood ${ }^{24, l}$, K. Gregersen ${ }^{35}$, I.M. Gregor ${ }^{41}$, P. Grenier ${ }^{143}$, J. Griffiths ${ }^{138}$, N. Grigalashvili64, A.A. Grillo ${ }^{137}$, S. Grinstein ${ }^{11}$, Y.V. Grishkevich ${ }^{97}$, J.-F. Grivaz ${ }^{115}$, M. Groh ${ }^{99}$, E. Gross ${ }^{172}$, J. GrosseKnetter $^{54}$, J. Groth-Jensen ${ }^{172}$, K. Grybel ${ }^{141}$, V.J. Guarino ${ }^{5}$, D. Guest ${ }^{176}$, C. Guicheney ${ }^{33}$, A. Guida ${ }^{72 a, 72 b}$, S. Guindon ${ }^{54}$, H. Guler ${ }^{85, n}$, J. Gunther ${ }^{125}$, B. Guo ${ }^{158}$, J. Guo ${ }^{34}$, A. Gupta ${ }^{30}$, Y. Gusakov ${ }^{64}$, V.N. Gushchin ${ }^{128}$, P. Gutierrez ${ }^{111}$, N. Guttman ${ }^{153}$, O. Gutzwiller ${ }^{173}$, C. Guyot ${ }^{136}$, C. Gwenlan ${ }^{118}$, C.B. Gwilliam ${ }^{73}$, A. Haas ${ }^{143}$, S. Haas ${ }^{29}$, C. Haber ${ }^{14}$, H.K. Hadavand ${ }^{39}$, D.R. Hadley ${ }^{17}$, P. Haefner ${ }^{99}$, F. Hahn ${ }^{29}$, S. Haider ${ }^{29}$, Z. Hajduk ${ }^{38}$, H. Hakobyan ${ }^{177}$, D. Hall ${ }^{118}$, J. Haller ${ }^{54}$, K. Hamacher ${ }^{175}$, P. Hamal ${ }^{113}$, M. Hamer ${ }^{54}$, A. Hamilton ${ }^{145 b, o}$, S. Hamilton ${ }^{161}$, H. Han ${ }^{32 a}$, L. Han ${ }^{32 b}$, K. Hanagaki ${ }^{116}$, K. Hanawa ${ }^{160}$, M. Hance ${ }^{14}$, C. Handel ${ }^{81}$, P. Hanke ${ }^{58 a}$, J.R. Hansen ${ }^{35}$, J.B. Hansen ${ }^{35}$,

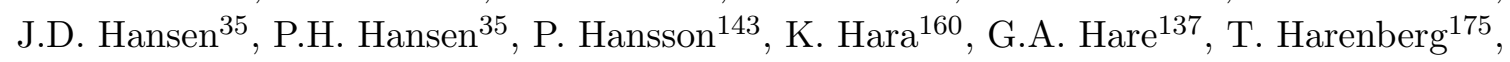
S. Harkusha ${ }^{90}$, D. Harper ${ }^{87}$, R.D. Harrington ${ }^{45}$, O.M. Harris ${ }^{138}$, K. Harrison ${ }^{17}$, J. Hartert ${ }^{48}$, F. Hartjes ${ }^{105}$, T. Haruyama ${ }^{65}$, A. Harvey ${ }^{56}$, S. Hasegawa ${ }^{101}$, Y. Hasegawa ${ }^{140}$, S. Hassani ${ }^{136}$, M. Hatch ${ }^{29}$, D. Hauff ${ }^{99}$, S. Haug ${ }^{16}$, M. Hauschild ${ }^{29}$, R. Hauser ${ }^{88}$, M. Havranek ${ }^{20}$, B.M. Hawes ${ }^{118}$, C.M. Hawkes ${ }^{17}$, R.J. Hawkings ${ }^{29}$, A.D. Hawkins ${ }^{79}$, D. Hawkins ${ }^{163}$, T. Hayakawa ${ }^{66}$, T. Hayashi ${ }^{160}$, D. Hayden ${ }^{76}$, H.S. Hayward ${ }^{73}$, S.J. Haywood ${ }^{129}$, E. Hazen ${ }^{21}$, M. He ${ }^{32 d}$, S.J. Head ${ }^{17}$, V. Hedberg ${ }^{79}$, L. Heelan ${ }^{7}$, S. Heim ${ }^{88}$, B. Heinemann ${ }^{14}$, S. Heisterkamp ${ }^{35}$, L. Helary ${ }^{4}$, C. Heller ${ }^{98}$, M. Heller ${ }^{29}$, S. Hellman 146a,146b D. Hellmich ${ }^{20}$, C. Helsens ${ }^{11}$, R.C.W. Henderson ${ }^{71}$, M. Henke ${ }^{58 a}$, A. Henrichs ${ }^{54}$, A.M. Henriques Correia ${ }^{29}$, S. Henrot-Versille ${ }^{115}$, F. Henry-Couannier ${ }^{83}$, C. Hensel ${ }^{54}$, T. Henß ${ }^{175}$, C.M. Hernandez ${ }^{7}$, Y. Hernández Jiménez ${ }^{167}$, R. Herrberg ${ }^{15}$, A.D. Hershenhorn ${ }^{152}$, G. Herten ${ }^{48}$, R. Hertenberger ${ }^{98}$, L. Hervas ${ }^{29}$, G.G. Hesketh ${ }^{77}$, N.P. Hessey ${ }^{105}$, E. HigónRodriguez $^{167}$, D. Hill ${ }^{5, *}$, J.C. Hill ${ }^{27}$, N. Hill ${ }^{5}$, K.H. Hiller ${ }^{41}$, S. Hillert ${ }^{20}$, S.J. Hillier ${ }^{17}$, I. Hinchliffe ${ }^{14}$, E. Hines ${ }^{120}$, M. Hirose ${ }^{116}$, F. Hirsch ${ }^{42}$, D. Hirschbuehl ${ }^{175}$, J. Hobbs ${ }^{148}$, N. $\operatorname{Hod}^{153}$, M.C. Hodgkinson ${ }^{139}$, P. Hodgson ${ }^{139}$, A. Hoecker ${ }^{29}$, M.R. Hoeferkamp ${ }^{103}$,

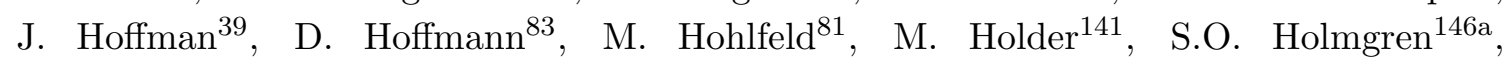
T. Holy ${ }^{127}$, J.L. Holzbauer ${ }^{88}$, Y. Homma ${ }^{66}$, T.M. Hong ${ }^{120}$, L. Hooft van Huysduynen ${ }^{108}$, 
T. Horazdovsky ${ }^{127}$, C. Horn ${ }^{143}$, S. Horner ${ }^{48}$, J-Y. Hostachy ${ }^{55}$, S. Hou ${ }^{151}$, M.A. Houlden ${ }^{73}$,

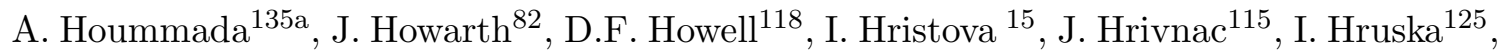
T. Hryn'ova ${ }^{4}$, P.J. Hsu ${ }^{81}$, S.-C. Hsu ${ }^{14}$, G.S. Huang ${ }^{111}$, Z. Hubacek ${ }^{127}$, F. Hubaut ${ }^{83}$,

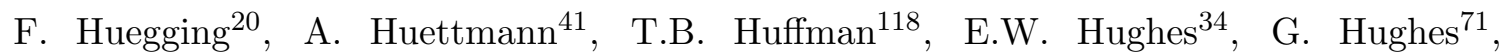
R.E. Hughes-Jones ${ }^{82}$, M. Huhtinen ${ }^{29}$, P. Hurst ${ }^{57}$, M. Hurwitz ${ }^{14}$, U. Husemann ${ }^{41}$,

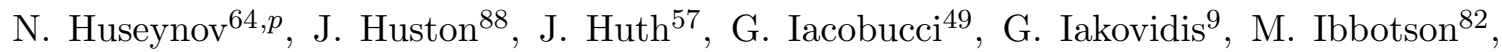
I. Ibragimov ${ }^{141}$, R. Ichimiya ${ }^{66}$, L. Iconomidou-Fayard ${ }^{115}$, J. Idarraga ${ }^{115}$, P. Iengo ${ }^{102 a}$, O. Igonkina ${ }^{105}$, Y. Ikegami ${ }^{65}$, M. Ikeno ${ }^{65}$, Y. Ilchenko ${ }^{39}$, D. Iliadis ${ }^{154}$, N. Ilic ${ }^{158}$, M. Imori ${ }^{155}$, T. Ince ${ }^{20}, J_{\text {. Inigo-Golfin }}{ }^{29}$, P. Ioannou ${ }^{8}$, M. Iodice ${ }^{134 a}$, V. Ippolito ${ }^{132 a, 132 b}$, A. Irles Quiles ${ }^{167}$, C. Isaksson ${ }^{166}$, A. Ishikawa ${ }^{66}$, M. Ishino ${ }^{67}$, R. Ishmukhametov ${ }^{39}$, C. Issever ${ }^{118}$, S. Istin ${ }^{18 a}$, A.V. Ivashin ${ }^{128}$, W. Iwanski ${ }^{38}$, H. Iwasaki ${ }^{65}$, J.M. Izen ${ }^{40}$, V. Izzo ${ }^{102 a}$, B. Jackson ${ }^{120}$, J.N. Jackson ${ }^{73}$, P. Jackson ${ }^{143}$, M.R. Jaekel ${ }^{29}$, V. Jain ${ }^{60}$, K. Jakobs ${ }^{48}$, S. Jakobsen ${ }^{35}$, J. Jakubek ${ }^{127}$, D.K. Jana ${ }^{111}$, E. Jankowski ${ }^{158}$, E. Jansen ${ }^{77}$, H. Jansen ${ }^{29}$, A. Jantsch ${ }^{99}$, M. Janus ${ }^{20}$, G. Jarlskog ${ }^{79}$, L. Jeanty ${ }^{57}$, K. Jelen ${ }^{37}$, I. JenLa Plante ${ }^{30}$, P. Jenni ${ }^{29}$, A. Jeremie ${ }^{4}$, P. Jež ${ }^{35}$, S. Jézéquel ${ }^{4}$, M.K. Jha ${ }^{19 a}$, H. Ji ${ }^{173}$, W. Ji ${ }^{81}$, J. Jia ${ }^{148}$, Y. Jiang ${ }^{32 b}$, M. Jimenez Belenguer ${ }^{41}$, G. Jin ${ }^{32 b}, \quad$ S. Jin ${ }^{32 a}$, O. Jinnouchi ${ }^{157}$, M.D. Joergensen ${ }^{35}$, D. Joffe ${ }^{39}$, L.G. Johansen ${ }^{13}$, M. Johansen ${ }^{146 a, 146 b}$, K.E. Johansson ${ }^{146 a}$, P. Johansson ${ }^{139}$, S. Johnert ${ }^{41}$, K.A. Johns ${ }^{6}$, K. Jon-And ${ }^{146 a, 146 b}$, G. Jones ${ }^{118}$, R.W.L. Jones ${ }^{71}$, T.W. Jones ${ }^{77}$, T.J. Jones ${ }^{73}$, O. Jonsson ${ }^{29}$, C. Joram ${ }^{29}$, P.M. Jorge ${ }^{124 a}$, J. Joseph ${ }^{14}$, J. Jovicevic ${ }^{147}$, T. Jovin ${ }^{12 b}$, X. Ju ${ }^{173}$, C.A. Jung ${ }^{42}$, R.M. Jungst ${ }^{29}$, V. Juranek ${ }^{125}$, P. Jussel ${ }^{61}$, A. Juste Rozas ${ }^{11}$, V.V. Kabachenko ${ }^{128}$, S. Kabana ${ }^{16}$, M. Kaci ${ }^{167}$, A. Kaczmarska ${ }^{38}$, P. Kadlecik ${ }^{35}$, M. Kado ${ }^{115}$, H. Kagan ${ }^{109}$, M. Kagan ${ }^{57}$, S. Kaiser ${ }^{99}$, E. Kajomovitz ${ }^{152}$, S. Kalinin ${ }^{175}$, L.V. Kalinovskaya ${ }^{64}$, S. Kama ${ }^{39}$, N. Kanaya ${ }^{155}$, M. Kaneda ${ }^{29}$, S. Kaneti ${ }^{27}$, T. Kanno ${ }^{157}$, V.A. Kantserov ${ }^{96}$, J. Kanzaki ${ }^{65}$, B. Kaplan ${ }^{176}$, A. Kapliy ${ }^{30}$, J. Kaplon ${ }^{29}$, D. Kar ${ }^{43}$, M. Karagounis ${ }^{20}$, M. Karagoz ${ }^{118}$, M. Karnevskiy ${ }^{41}$, K. Karr ${ }^{5}$, V. Kartvelishvili ${ }^{71}$, A.N. Karyukhin ${ }^{128}$, L. Kashif ${ }^{173}$, G. Kasieczka ${ }^{58 b}$, R.D. Kass ${ }^{109}$, A. Kastanas ${ }^{13}$, M. Kataoka ${ }^{4}$, Y. Kataoka ${ }^{155}$, E. Katsoufis ${ }^{9}$, J. Katzy ${ }^{41}$, V. Kaushik ${ }^{6}$, K. Kawagoe ${ }^{66}$, T. Kawamoto ${ }^{155}$, G. Kawamura ${ }^{81}$, M.S. Kayl ${ }^{105}$, V.A. Kazanin ${ }^{107}$, M.Y. Kazarinov ${ }^{64}$, R. Keeler ${ }^{169}$, R. Kehoe ${ }^{39}$, M. Keil ${ }^{54}$, G.D. Kekelidze ${ }^{64}$, J. Kennedy ${ }^{98}$, C.J. Kenney ${ }^{143}$, M. Kenyon ${ }^{53}$, O. Kepka ${ }^{125}$, N. Kerschen ${ }^{29}$, B.P. Kerševan ${ }^{74}$, S. Kersten ${ }^{175}$, K. Kessoku ${ }^{155}$, J. Keung ${ }^{158}$, F. Khalil-zada ${ }^{10}$, H. Khandanyan ${ }^{165}$, A. Khanov ${ }^{112}$, D. Kharchenko ${ }^{64}$, A. Khodinov ${ }^{96}$, A.G. Kholodenko ${ }^{128}$, A. Khomich ${ }^{58 a}$, T.J. Khoo ${ }^{27}$, G. Khoriauli ${ }^{20}$, A. Khoroshilov ${ }^{175}$, N. Khovanskiy ${ }^{64}$, V. Khovanskiy ${ }^{95}$, E. Khramov ${ }^{64}$, J. Khubua ${ }^{51 b}$, H. Kim ${ }^{146 a, 146 b}$, M.S. Kim ${ }^{2}$, S.H. Kim ${ }^{160}$, N. Kimura ${ }^{171}$, O. Kind ${ }^{15}$, B.T. King ${ }^{73}$, M. King ${ }^{66}$, R.S.B. King ${ }^{118}$, J. Kirk ${ }^{129}$, L.E. Kirsch ${ }^{22}$, A.E. Kiryunin ${ }^{99}$, T. Kishimoto ${ }^{66}$, D. Kisielewska ${ }^{37}$, T. Kittelmann ${ }^{123}$, A.M. Kiver ${ }^{128}$, E. Kladiva ${ }^{144 \mathrm{~b}}$, J. Klaiber-Lodewigs ${ }^{42}$, M. Klein ${ }^{73}$, U. Klein ${ }^{73}$, K. Kleinknecht ${ }^{81}$, M. Klemetti ${ }^{85}$, A. Klier ${ }^{172}$, P. Klimek ${ }^{146 a, 146 b}$, A. Klimentov ${ }^{24}$, R. Klingenberg ${ }^{42}$, J.A. Klinger ${ }^{82}$, E.B. Klinkby ${ }^{35}$, T. Klioutchnikova ${ }^{29}$, P.F. Klok ${ }^{104}$, S. Klous ${ }^{105}$, E.E. Kluge ${ }^{58 a}$, T. Kluge ${ }^{73}$, P. Kluit ${ }^{105}$, S. Kluth ${ }^{99}$, N.S. Knecht ${ }^{158}$, E. Kneringer ${ }^{61}$, J. Knobloch ${ }^{29}$, E.B.F.G. Knoops ${ }^{83}$, A. Knue ${ }^{54}$, B.R. Ko ${ }^{44}$, T. Kobayashi ${ }^{155}$, M. Kobel $^{43}$, M. Kocian ${ }^{143}$, P. Kodys ${ }^{126}$, K. Köneke ${ }^{29}$, A.C. König ${ }^{104}$, S. Koenig ${ }^{81}$, L. Köpke ${ }^{81}$, F. Koetsveld ${ }^{104}$, P. Koevesarki ${ }^{20}$, T. Koffas ${ }^{28}$, E. Koffeman ${ }^{105}$, L.A. Kogan ${ }^{118}$, 
F. Kohn ${ }^{54}$, Z. Kohout ${ }^{127}$, T. Kohriki ${ }^{65}$, T. Koi ${ }^{143}$, T. Kokott ${ }^{20}$, G.M. Kolachev ${ }^{107}$, H. Kolanoski ${ }^{15}$, V. Kolesnikov ${ }^{64}$, I. Koletsou ${ }^{89 a}$, J. Koll ${ }^{88}$, M. Kollefrath ${ }^{48}$, S.D. Kolya ${ }^{82}$, A.A. Komar ${ }^{94}$, Y. Komori ${ }^{155}$, T. Kondo ${ }^{65}$, T. Kono ${ }^{41, q}$, A.I. Kononov ${ }^{48}$, R. Konoplich ${ }^{108, r}$,

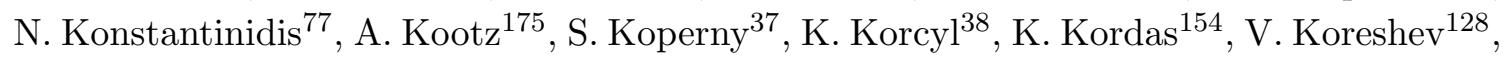
A. Korn ${ }^{118}$, A. Korol ${ }^{107}$, I. Korolkov ${ }^{11}$, E.V. Korolkova ${ }^{139}$, V.A. Korotkov ${ }^{128}$, O. Kortner ${ }^{99}$, S. Kortner ${ }^{99}$, V.V. Kostyukhin ${ }^{20}$, M.J. Kotamäkii ${ }^{29}$, S. Kotov ${ }^{99}$, V.M. Kotov ${ }^{64}$, A. Kotwal ${ }^{44}$, C. Kourkoumelis ${ }^{8}$, V. Kouskoura ${ }^{154}$, A. Koutsman ${ }^{159 a}$, R. Kowalewski ${ }^{169}$, T.Z. Kowalski ${ }^{37}$, W. Kozanecki ${ }^{136}$, A.S. Kozhin ${ }^{128}$, V. Kral ${ }^{127}$, V.A. Kramarenko ${ }^{97}$, G. Kramberger ${ }^{74}$, M.W. Krasny ${ }^{78}$, A. Krasznahorkay ${ }^{108}$, J. Kraus ${ }^{88}$, J.K. Kraus ${ }^{20}$, A. Kreisel ${ }^{153}$, F. Krejci ${ }^{127}$, J. Kretzschmar ${ }^{73}$, N. Krieger ${ }^{54}$, P. Krieger ${ }^{158}$, K. Kroeninger ${ }^{54}$, H. Kroha ${ }^{99}$, J. Kroll ${ }^{120}$, J. Kroseberg ${ }^{20}$, J. Krstic ${ }^{12 a}$, U. Kruchonak ${ }^{64}$, H. Krüger ${ }^{20}$, T. Kruker ${ }^{16}$, N. Krumnack ${ }^{63}$, Z.V. Krumshteyn ${ }^{64}$, A. Kruth ${ }^{20}$, T. Kubota ${ }^{86}$, S. Kuday ${ }^{3 a}$, S. Kuehn ${ }^{48}$, A. Kugel ${ }^{58 c}$, T. Kuhl ${ }^{41}$, D. Kuhn ${ }^{61}$, V. Kukhtin ${ }^{64}$, Y. Kulchitsky ${ }^{90}$, S. Kuleshov ${ }^{31 b}$, C. Kummer ${ }^{98}$, M. Kuna ${ }^{78}$, N. Kundu ${ }^{118}$, J. Kunkle ${ }^{120}$, A. Kupco ${ }^{125}$, H. Kurashige ${ }^{66}$, M. Kurata ${ }^{160}$, Y.A. Kurochkin ${ }^{90}$, V. Kus ${ }^{125}$, E.S. Kuwertz ${ }^{147}$, M. Kuze ${ }^{157}$, J. Kvita ${ }^{142}$, R. Kwee ${ }^{15}$, A. La Rosa ${ }^{49}$, L. La Rotonda ${ }^{36 a, 36 b}$, L. Labarga ${ }^{80}$, J. Labbe ${ }^{4}$, S. Lablak ${ }^{135 a}$, C. Lacasta ${ }^{167}$, F. Lacava ${ }^{132 a, 132 b}$, H. Lacker ${ }^{15}$, D. Lacour ${ }^{78}$, V.R. Lacuesta ${ }^{167}$, E. Ladygin ${ }^{64}$, R. Lafaye ${ }^{4}$, B. Laforge ${ }^{78}$, T. Lagouri ${ }^{80}$, S. Lai ${ }^{48}$, E. Laisne ${ }^{55}$, M. Lamanna ${ }^{29}$, C.L. Lampen ${ }^{6}$, W. Lampl ${ }^{6}$, E. Lancon ${ }^{136}$, U. Landgraf ${ }^{48}$, M.P.J. Landon ${ }^{75}$, J.L. Lane ${ }^{82}$, C. Lange ${ }^{41}$, A.J. Lankford ${ }^{163}$, F. Lanni ${ }^{24}$, K. Lantzsch $^{175}$, S. Laplace ${ }^{78}$, C. Lapoire ${ }^{20}$, J.F. Laporte ${ }^{136}$, T. Lari ${ }^{89 a}$, A.V. Larionov ${ }^{128}$, A. Larner ${ }^{118}$, C. Lasseur ${ }^{29}$, M. Lassnig ${ }^{29}$, P. Laurelli ${ }^{47}$, V. Lavorini ${ }^{36 a, 36 b}$, W. Lavrijsen ${ }^{14}$, P. Laycock ${ }^{73}$, A.B. Lazarev ${ }^{64}$, O. Le Dortz ${ }^{78}$, E. Le Guirriec ${ }^{83}$, C. Le Maner ${ }^{158}$, E. Le Menedeu ${ }^{9}$, C. Lebel ${ }^{93}$, T. LeCompte ${ }^{5}$, F. Ledroit-Guillon ${ }^{55}$, H. Lee ${ }^{105}$, J.S.H. Lee ${ }^{116}$, S.C. Lee ${ }^{151}$, L. Lee ${ }^{176}$, M. Lefebvre ${ }^{169}$, M. Legendre ${ }^{136}$, A. Leger ${ }^{49}$, B.C. LeGeyt ${ }^{120}$, F. Legger ${ }^{98}$, C. Leggett ${ }^{14}$, M. Lehmacher ${ }^{20}$, G. Lehmann Miotto ${ }^{29}$, X. Lei ${ }^{6}$, M.A.L. Leite ${ }^{23 d}$, R. Leitner ${ }^{126}$, D. Lellouch ${ }^{172}$, M. Leltchouk ${ }^{34}$, B. Lemmer ${ }^{54}$, V. Lendermann ${ }^{58 a}$, K.J.C. Leney ${ }^{145 b}$, T. Lenz ${ }^{105}$, G. Lenzen ${ }^{175}$, B. Lenzi ${ }^{29}$, K. Leonhardt ${ }^{43}$, S. Leontsinis ${ }^{9}$, C. Leroy ${ }^{93}$, JR. Lessard ${ }^{169}$, J. Lesser ${ }^{146 a}$, C.G. Lester ${ }^{27}$, A. Leung Fook Cheong ${ }^{173}$, J. Levêque ${ }^{4}$, D. Levin ${ }^{87}$, L.J. Levinson ${ }^{172}$, M.S. Levitski ${ }^{128}$, A. Lewis ${ }^{118}$, G.H. Lewis ${ }^{108}$, A.M. Leyko ${ }^{20}$, M. Leyton ${ }^{15}$, B. $\mathrm{Li}^{83}$, H. $\mathrm{Li}^{173, s}, \mathrm{~S} . \mathrm{Li}^{32 \mathrm{~b}, t}, \mathrm{X} . \mathrm{Li}^{87}$, Z. Liang ${ }^{118, u}, \mathrm{H}_{\mathrm{Liao}}{ }^{33}$, B. Liberti ${ }^{133 a}$, P. Lichard $^{29}$, M. Lichtnecker ${ }^{98}$, K. Lie ${ }^{165}$, W. Liebig ${ }^{13}$, R. Lifshitz ${ }^{152}$, C. Limbach $^{20}$, A. Limosani ${ }^{86}$, M. Limper ${ }^{62}$, S.C. Lin ${ }^{151, v}$, F. Linde ${ }^{105}$, J.T. Linnemann ${ }^{88}$, E. Lipeles ${ }^{120}$, L. Lipinsky ${ }^{125}$, A. Lipniacka ${ }^{13}$, T.M. Liss ${ }^{165}$, D. Lissauer ${ }^{24}$, A. Lister ${ }^{49}$, A.M. Litke ${ }^{137}$, C. $\mathrm{Liu}^{28}$, D. $\mathrm{Liu}^{151}$, H. Liu ${ }^{87}$, J.B. Liu ${ }^{87}$, M. Liu ${ }^{32 b}$, Y. Liu ${ }^{32 b}$, M. Livan ${ }^{119 a, 119 b}$, S.S.A. Livermore ${ }^{118}$, A. Lleres $^{55}$, J. Llorente Merino ${ }^{80}$, S.L. Lloyd ${ }^{75}$, E. Lobodzinska ${ }^{41}$, P. Loch $^{6}$, W.S. Lockman ${ }^{137}$, T. Loddenkoetter ${ }^{20}$, F.K. Loebinger ${ }^{82}$, A. Loginov ${ }^{176}$, C.W. Loh $^{168}$, T. Lohse ${ }^{15}$, K. Lohwasser ${ }^{48}$, M. Lokajicek ${ }^{125}$, J. Loken ${ }^{118}$, V.P. Lombardo ${ }^{4}$, R.E. Long ${ }^{71}$, L. Lopes ${ }^{124 a}$, D. Lopez Mateos ${ }^{57}$, J. Lorenz ${ }^{98}$, N. Lorenzo Martinez ${ }^{115}$, M. Losada ${ }^{162}$, P. Loscutoff ${ }^{14}$, F. Lo Sterzo ${ }^{132 a, 132 b}$, M.J. Losty ${ }^{159 a}$,

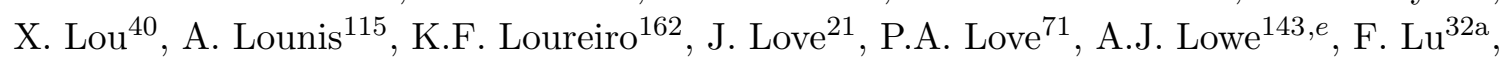
H.J. Lubatti ${ }^{138}$, C. Luci ${ }^{132 a, 132 b}$, A. Lucotte ${ }^{55}$, A. Ludwig ${ }^{43}$, D. Ludwig ${ }^{41}$, I. Ludwig ${ }^{48}$, J. Ludwig ${ }^{48}$, F. Luehring ${ }^{60}$, G. Luijckx ${ }^{105}$, D. Lumb $^{48}$, L. Luminari ${ }^{132 a}$, E. Lund ${ }^{117}$, 
B. Lund-Jensen ${ }^{147}$, B. Lundberg ${ }^{79}$, J. Lundberg ${ }^{146 a, 146 b}$, J. Lundquist ${ }^{35}$, M. Lungwitz ${ }^{81}$, G. Lutz ${ }^{99}$, D. Lynn $^{24}$, J. Lys ${ }^{14}$, E. Lytken ${ }^{79}$, H. Ma ${ }^{24}$, L.L. Ma ${ }^{173}$, J.A. Macana Goia ${ }^{93}$, G. Maccarrone ${ }^{47}$, A. Macchiolo ${ }^{99}$, B. Maček ${ }^{74}$, J. Machado Miguensi ${ }^{124 a}$, R. Mackeprang ${ }^{35}$, R.J. Madaras ${ }^{14}$, W.F. Mader ${ }^{43}$, R. Maenner ${ }^{58 c}$, T. Maeno ${ }^{24}$, P. Mättig ${ }^{175}$, S. Mättig ${ }^{41}$, L. Magnoni ${ }^{29}$, E. Magradze $^{54}$, Y. Mahalalel ${ }^{153}$, K. Mahboubi ${ }^{48}$, G. Mahout ${ }^{17}$, C. Maiani ${ }^{132 a, 132 b}$, C. Maidantchik ${ }^{23 a}$, A. Maio ${ }^{124 a, b}$, S. Majewski ${ }^{24}$, Y. Makida ${ }^{65}$, N. Makovec ${ }^{115}$, P. Mal ${ }^{136}$, B. Malaescu ${ }^{29}$, Pa. Malecki ${ }^{38}$, P. Malecki ${ }^{38}$, V.P. Maleev ${ }^{121}$, F. Malek ${ }^{55}$, U. Mallik ${ }^{62}$, D. Malon ${ }^{5}$, C. Malone ${ }^{143}$, S. Maltezos ${ }^{9}$, V. Malyshev ${ }^{107}$, S. Malyukov ${ }^{29}$, R. Mameghani ${ }^{98}$, J. Mamuzic ${ }^{12 b}$, A. Manabe ${ }^{65}$, L. Mandelli ${ }^{89 a}$, I. Mandić ${ }^{74}$, R. Mandrysch ${ }^{15}$, J. Maneira ${ }^{124 a}$, P.S. Mangeard ${ }^{88}$, L. Manhaes de Andrade Filho ${ }^{23 a}$, I.D. Manjavidze ${ }^{64}$, A. Mann ${ }^{54}$, P.M. Manning ${ }^{137}$, A. ManousakisKatsikakis $^{8}$, B. Mansoulie ${ }^{136}$, A. Manz ${ }^{99}$, A. Mapelli ${ }^{29}$, L. Mapelli ${ }^{29}$, L. March ${ }^{80}$, J.F. Marchand ${ }^{28}$, F. Marchese ${ }^{133 a, 133 b}$, G. Marchiori ${ }^{78}$, M. Marcisovsky ${ }^{125}$, A. Marin ${ }^{21, *}$, C.P. Marino ${ }^{169}$, F. Marroquim ${ }^{23 a}$, R. Marshall ${ }^{82}$, Z. Marshall ${ }^{29}$, F.K. Martens ${ }^{158}$, S. Marti-

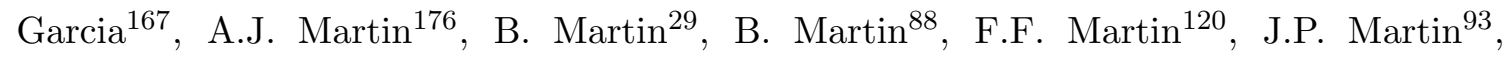
Ph. Martin ${ }^{55}$, T.A. Martin ${ }^{17}$, V.J. Martin ${ }^{45}$, B. Martin dit Latour ${ }^{49}$, S. MartinHaugh $^{149}$, M. Martinez ${ }^{11}$, V. Martinez Outschoorn ${ }^{57}$, A.C. Martyniuk ${ }^{169}$, M. Marx ${ }^{82}$, F. Marzano ${ }^{132 a}$, A. Marzin ${ }^{111}$, L. Masetti ${ }^{81}$, T. Mashimo ${ }^{155}$, R. Mashinistov ${ }^{94}$, J. Masik ${ }^{82}$, A.L. Maslennikov ${ }^{107}$, I. Massa ${ }^{19 a, 19 b}$, G. Massaro ${ }^{105}$, N. Massol ${ }^{4}$, P. Mastrandrea ${ }^{132 a, 132 b}$, A. Mastroberardino ${ }^{36 a, 36 b}$, T. Masubuchi ${ }^{155}$, M. Mathes ${ }^{20}$, P. Matricon ${ }^{115}$, H. Matsumoto ${ }^{155}$, H. Matsunaga ${ }^{155}$, T. Matsushita ${ }^{66}$, C. Mattravers ${ }^{118, c}$, J.M. Maugain ${ }^{29}$, J. Maurer ${ }^{83}$, S.J. Maxfield ${ }^{73}$, D.A. Maximov ${ }^{107, f}$, E.N. May ${ }^{5}$, A. Mayne ${ }^{139}$, R. Mazini ${ }^{151}$, M. Mazur ${ }^{20}$, M. Mazzanti ${ }^{89 a}$, E. Mazzoni122a,122b, S.P. Mc Kee ${ }^{87}$, A. McCarn ${ }^{165}$, R.L. McCarthy ${ }^{148}$, T.G. McCarthy ${ }^{28}$, N.A. McCubbin ${ }^{129}$, K.W. McFarlane ${ }^{56}$, J.A. Mcfayden ${ }^{139}$, H. McGlone ${ }^{53}$, G. Mchedlidze ${ }^{51 b}$, R.A. McLaren ${ }^{29}$, T. Mclaughlan ${ }^{17}$, S.J. McMahon ${ }^{129}$, R.A. McPherson ${ }^{169, j}$, A. Meade ${ }^{84}$, J. Mechnich ${ }^{105}$, M. Mechtel ${ }^{175}$, M. Medinnis ${ }^{41}$, R. Meera-Lebbai ${ }^{111}$, T. Meguro ${ }^{116}$, R. Mehdiyev ${ }^{93}$, S. Mehlhase $^{35}$, A. Mehta ${ }^{73}$, K. Meier ${ }^{58 a}$, B. Meirose ${ }^{79}$, C. Melachrinos ${ }^{30}$, B.R. Mellado Garcia ${ }^{173}$, L. Mendoza Navas ${ }^{162}$, Z. Meng ${ }^{151, s}$, A. Mengarelli19a,19b, S. Menke ${ }^{99}$, C. Menot $^{29}$, E. Meoni ${ }^{11}$, K.M. Mercurio ${ }^{57}$, P. Mermod ${ }^{49}$, L. Merola ${ }^{102 a, 102 b}$, C. Meroni ${ }^{89 a}$, F.S. Merritt ${ }^{30}$, H. Merritt ${ }^{109}$, A. Messina ${ }^{29}$, J. Metcalfe ${ }^{103}$, A.S. Mete ${ }^{63}$, C. Meyer ${ }^{81}$, C. Meyer $^{30}$, J-P. Meyer ${ }^{136}$, J. Meyer ${ }^{174}$, J. Meyer ${ }^{54}$, T.C. Meyer ${ }^{29}$, W.T. Meyer ${ }^{63}$,

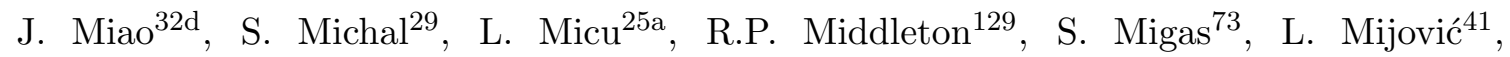
G. Mikenberg ${ }^{172}$, M. Mikestikova ${ }^{125}$, M. Mikuž ${ }^{74}$, D.W. Miller ${ }^{30}$, R.J. Miller ${ }^{88}$, W.J. Mills ${ }^{168}$, C. Mills ${ }^{57}$, A. Milov ${ }^{172}$, D.A. Milstead ${ }^{146 a, 146 b}$, D. Milstein ${ }^{172}$, A.A. Minaenko ${ }^{128}$, M. Miñano Moya ${ }^{167}$, I.A. Minashvili ${ }^{64}$, A.I. Mincer ${ }^{108}$, B. Mindur ${ }^{37}$, M. Mineev ${ }^{64}$, Y. Ming ${ }^{173}$, L.M. Mir ${ }^{11}$, G. Mirabelli ${ }^{132 a}$, L. Miralles Verge ${ }^{11}$, A. Misiejuk ${ }^{76}$, J. Mitrevski ${ }^{137}$, G.Y. Mitrofanov ${ }^{128}$, V.A. Mitsou ${ }^{167}$, S. Mitsui ${ }^{65}$, P.S. Miyagawa ${ }^{139}$, K. Miyazaki ${ }^{66}$, J.U. Mjörnmark ${ }^{79}$, T. Moa ${ }^{146 a, 146 b}$, P. Mockett ${ }^{138}$, S. Moed ${ }^{57}$, V. Moeller ${ }^{27}$, K. Mönig ${ }^{41}$, N. Möser ${ }^{20}$, S. Mohapatra ${ }^{148}$, W. Mohr ${ }^{48}$, S. Mohrdieck-Möck ${ }^{99}$, A.M. Moisseev ${ }^{128, *}$, R. Moles-Valls ${ }^{167}$, J. Molina-Perez ${ }^{29}$, J. Monk $^{77}$, E. Monnier ${ }^{83}$, S. Montesano ${ }^{89 a, 89 b}$, F. Monticelli ${ }^{70}$, S. Monzani19a,19b , R.W. Moore ${ }^{2}$, G.F. Moorhead ${ }^{86}$, C. Mora Herrera ${ }^{49}$, A. Moraes ${ }^{53}$, N. Morange ${ }^{136}$, J. Morel ${ }^{54}$, 
G. Morello ${ }^{36 a, 36 b}$, D. Moreno ${ }^{81}$, M. Moreno Llácer ${ }^{167}$, P. Morettini ${ }^{50 a}$, M. Morgenstern ${ }^{43}$, M. Morii ${ }^{57}$, J. Morin ${ }^{75}$, A.K. Morley ${ }^{29}$, G. Mornacchi ${ }^{29}$, S.V. Morozov ${ }^{96}$, J.D. Morris ${ }^{75}$, L. Morvaj101, H.G. Moser ${ }^{99}$, M. Mosidze ${ }^{51 b}$, J. Moss ${ }^{109}$, R. Mount ${ }^{143}$, E. Mountricha ${ }^{9, w}$, S.V. Mouraviev ${ }^{94}$, E.J.W. Moyse ${ }^{84}$, M. Mudrinic ${ }^{12 b}$, F. Mueller ${ }^{58 a}$, J. Mueller ${ }^{123}$, K. Mueller ${ }^{20}$, T.A. Müller ${ }^{98}$, T. Mueller ${ }^{81}$, D. Muenstermann ${ }^{29}$, A. Muir ${ }^{168}$, Y. Munwes ${ }^{153}$, W.J. Murray ${ }^{129}$, I. Mussche ${ }^{105}$, E. Musto102a,102b , A.G. Myagkov ${ }^{128}$, M. Myska ${ }^{125}$, J. Nadal ${ }^{11}$, K. Nagai ${ }^{160}$, K. Nagano ${ }^{65}$, A. Nagarkar ${ }^{109}$, Y. Nagasaka ${ }^{59}$, M. Nagel ${ }^{99}$, A.M. Nairz ${ }^{29}$, Y. Nakahama ${ }^{29}$, K. Nakamura ${ }^{155}$, T. Nakamura ${ }^{155}$, I. Nakano ${ }^{110}$, G. Nanava ${ }^{20}$, A. Napier ${ }^{161}$, R. Narayan ${ }^{58 b}$, M. Nash ${ }^{77, c}$, N.R. Nation ${ }^{21}$, T. Nattermann ${ }^{20}$, T. Naumann ${ }^{41}$, G. Navarro ${ }^{162}$, H.A. $\mathrm{Neal}^{87}$, E. $\mathrm{Nebot}^{80}$, P.Yu. Nechaeva ${ }^{94}$, T.J. Neep ${ }^{82}$, A. Negri ${ }^{119 a, 119 b}$, G. $\mathrm{Negri}^{29}$, S. Nektarijevic ${ }^{49}$, A. Nelson ${ }^{163}$, S. Nelson ${ }^{143}$, T.K. Nelson ${ }^{143}$, S. Nemecek ${ }^{125}$, P. Nemethy ${ }^{108}$, A.A. Nepomuceno ${ }^{23 a}$, M. Nessi ${ }^{29, x}$, M.S. Neubauer ${ }^{165}$, A. Neusiedl ${ }^{81}$, R.M. Neves ${ }^{108}$, P. Nevski ${ }^{24}$, P.R. Newman ${ }^{17}$, V. Nguyen Thi Hong ${ }^{136}$, R.B. Nickerson ${ }^{118}$, R. Nicolaidou ${ }^{136}$, L. Nicolas ${ }^{139}$, B. Nicquevert ${ }^{29}$, F. Niedercorn ${ }^{115}$, J. Nielsen ${ }^{137}$, T. Niinikoski ${ }^{29}$, N. Nikiforou ${ }^{34}$, A. Nikiforov ${ }^{15}$, V. Nikolaenko ${ }^{128}$, K. Nikolaev ${ }^{64}$, I. Nikolic-Audit ${ }^{78}$, K. Nikolics ${ }^{49}$, K. Nikolopoulos ${ }^{24}$, H. Nilsen ${ }^{48}$, P. Nilsson ${ }^{7}$, Y. Ninomiya ${ }^{155}$, A. Nisati ${ }^{132 a}$, T. Nishiyama ${ }^{66}$, R. Nisius ${ }^{99}$, L. Nodulman ${ }^{5}$, M. Nomachi ${ }^{116}$, I. Nomidis ${ }^{154}$, M. Nordberg ${ }^{29}$, B. Nordkvist ${ }^{146 a, 146 b}$, P.R. Norton ${ }^{129}$, J. Novakova ${ }^{126}$, M. Nozaki ${ }^{65}$, L. Nozka ${ }^{113}$, I.M. Nugent ${ }^{159 a}$, A.-E. Nuncio-Quiroz ${ }^{20}$, G. Nunes Hanninger ${ }^{86}$, T. Nunnemann ${ }^{98}$, E. Nurse ${ }^{77}$, B.J. O'Brien ${ }^{45}$, S.W. O'Neale ${ }^{17, *}$, D.C. O'Neil ${ }^{142}$, V. O'Shea ${ }^{53}$, L.B. Oakes ${ }^{98}$, F.G. Oakham ${ }^{28, d}$, H. Oberlack ${ }^{99}$, J. Ocariz ${ }^{78}$, A. Ochi ${ }^{66}$, S. Oda ${ }^{155}$, S. Odaka ${ }^{65}$, J. Odier ${ }^{83}$, H. Ogren ${ }^{60}$, A. $\mathrm{Oh}^{82}$, S.H. $\mathrm{Oh}^{44}$, C.C. Ohm ${ }^{146 a, 146 \mathrm{~b}}$, T. Ohshima ${ }^{101}$, H. Ohshita ${ }^{140}$, S. Okada ${ }^{66}$, H. Okawa ${ }^{163}$, Y. Okumura ${ }^{101}$, T. Okuyama ${ }^{155}$,

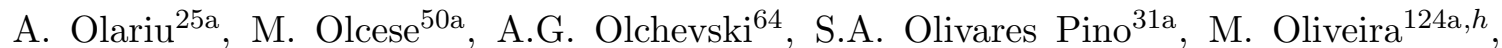
D. Oliveira Damazio ${ }^{24}$, E. Oliver Garcia ${ }^{167}$, D. Olivito ${ }^{120}$, A. Olszewski ${ }^{38}$, J. Olszowska $^{38}$, C. Omachi $^{66}$, A. Onofre ${ }^{124 a, y}$, P.U.E. Onyisi ${ }^{30}$, C.J. Oram ${ }^{159 a}$, M.J. Oreglia ${ }^{30}$, Y. Oren ${ }^{153}$, D. Orestano ${ }^{134 a, 134 b}$, I. Orlov ${ }^{107}$, C. Oropeza Barrera ${ }^{53}$, R.S. Orr ${ }^{158}$, B. Osculati50a,50b, R. Ospanov ${ }^{120}$, C. Osuna ${ }^{11}$, G. Otero y Garzon ${ }^{26}$, J.P. Ottersbach ${ }^{105}$, M. Ouchrif ${ }^{135 d}$, E.A. Ouellette ${ }^{169}$, F. Ould-Saada ${ }^{117}$, A. Ouraou ${ }^{136}$, Q. Ouyang ${ }^{32 a}$, A. Ovcharova ${ }^{14}$, M. Owen ${ }^{82}$, S. Owen ${ }^{139}$, V.E. Ozcan ${ }^{18 a}$, N. Ozturk ${ }^{7}$, A. Pacheco Pages ${ }^{11}$, C. Padilla Aranda ${ }^{11}$, S. Pagan Griso ${ }^{14}$, E. Paganis ${ }^{139}$, F. Paige ${ }^{24}$, P. Pais ${ }^{84}$, K. Pajchel ${ }^{117}$, G. Palacino ${ }^{159 b}$, C.P. Paleari ${ }^{6}$, S. Palestini ${ }^{29}$, D. Pallin ${ }^{33}$, A. Palma ${ }^{124 a}$, J.D. Palmer ${ }^{17}$, Y.B. Pan $^{173}$, E. Panagiotopoulou ${ }^{9}$, B. Panes ${ }^{31 a}$, N. Panikashvili ${ }^{87}$, S. Panitkin ${ }^{24}$, D. Pantea ${ }^{25 a}$, M. Panuskova ${ }^{125}$, V. Paolone ${ }^{123}$, A. Papadelis ${ }^{146 a}$, Th.D. Papadopoulou ${ }^{9}$, A. Paramonov ${ }^{5}$, D. Paredes Hernandez ${ }^{33}$, W. Park ${ }^{24, z}$, M.A. Parker ${ }^{27}$, F. Parodi50a,50b, J.A. Parsons ${ }^{34}$, U. Parzefall ${ }^{48}$, E. Pasqualucci ${ }^{132 a}$, S. Passaggio ${ }^{50 a}$, A. Passeri ${ }^{134 a}$, F. Pastore ${ }^{134 a, 134 b}$, Fr. Pastore ${ }^{76}$, G. Pásztor ${ }^{49, a a}$, S. Pataraia ${ }^{175}$, N. Patel ${ }^{150}$, J.R. Pater ${ }^{82}$, S. Patricelli102a,102b, T. Pauly ${ }^{29}$, M. Pecsy ${ }^{144 a}$, M.I. Pedraza Morales ${ }^{173}$, S.V. Peleganchuk ${ }^{107}$, H. Peng ${ }^{32 b}$, R. Pengo ${ }^{29}$, A. Penson ${ }^{34}$, J. Penwell ${ }^{60}$, M. Perantoni ${ }^{23 a}$, K. Perez ${ }^{34, a b}$, T. Perez Cavalcanti ${ }^{41}$, E. Perez Codina ${ }^{11}$, M.T. Pérez García-Estañ ${ }^{167}$, V. Perez Reale ${ }^{34}$, L. Perini ${ }^{89 a, 89 b}$, H. Pernegger ${ }^{29}$, R. Perrino ${ }^{72 a}$, P. Perrodo ${ }^{4}$, S. Persembe ${ }^{3 a}$, A. Perus ${ }^{115}$, V.D. Peshekhonov ${ }^{64}$, K. Peters ${ }^{29}$, B.A. Petersen ${ }^{29}$, J. Petersen ${ }^{29}$, T.C. Petersen ${ }^{35}$, E. Petit ${ }^{4}$, A. Petridis ${ }^{154}$, C. Petridou ${ }^{154}$, E. Petrolo ${ }^{132 a}$, 


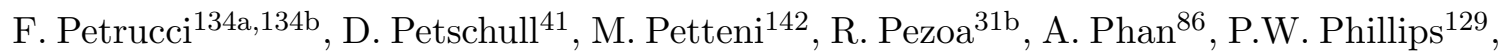
G. Piacquadio ${ }^{29}$, E. Piccaro ${ }^{75}$, M. Piccinini19a,19b, S.M. Piec ${ }^{41}$, R. Piegaia ${ }^{26}$, D.T. Pignotti ${ }^{109}$, J.E. Pilcher ${ }^{30}$, A.D. Pilkington ${ }^{82}$, J. Pina ${ }^{124 a, b}$, M. Pinamonti164a,164c, A. Pinder ${ }^{118}$, J.L. Pinfold ${ }^{2}$, J. Ping ${ }^{32 c}$, B. Pinto ${ }^{124 a}$, O. Pirotte ${ }^{29}$, C. Pizio ${ }^{89 a, 89 b}$, M. Plamondon ${ }^{169}$, M.-A. Pleier ${ }^{24}$, A.V. Pleskach ${ }^{128}$, A. Poblaguev ${ }^{24}$, S. Poddar ${ }^{58 a}$, F. Podlyski ${ }^{33}$, L. Poggioli ${ }^{115}$, T. Poghosyan ${ }^{20}$, M. Pohl ${ }^{49}$, F. Polci ${ }^{55}$, G. Polesello ${ }^{119 a}$, A. Policicchio ${ }^{36 a, 36 b}$, A. Polini ${ }^{19 a}$, J. Poll ${ }^{75}$, V. Polychronakos ${ }^{24}$, D.M. Pomarede ${ }^{136}$, D. Pomeroy $^{22}$, K. Pommès ${ }^{29}$, L. Pontecorvo ${ }^{132 a}$, B.G. Pope ${ }^{88}$, G.A. Popeneciu ${ }^{25 a}$, D.S. Popovic ${ }^{12 a}$, A. Poppleton ${ }^{29}$, X. Portell Bueso ${ }^{29}$, C. Posch ${ }^{21}$, G.E. Pospelov ${ }^{99}$, S. Pospisil ${ }^{127}$, I.N. Potrap ${ }^{99}$, C.J. Potter ${ }^{149}$, C.T. Potter ${ }^{114}$, G. Poulard ${ }^{29}$, J. Poveda ${ }^{173}$,

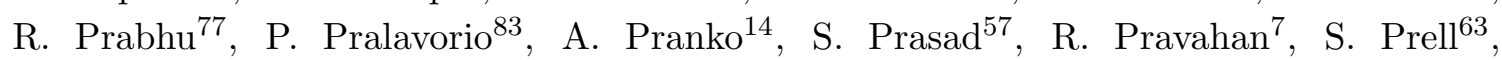
K. Pretzl $^{16}$, L. Pribyl ${ }^{29}$, D. Price ${ }^{60}$, J. Price ${ }^{73}$, L.E. Price ${ }^{5}$, M.J. Price ${ }^{29}$, D. Prieur ${ }^{123}$, M. Primavera ${ }^{72 a}$, K. Prokofiev ${ }^{108}$, F. Prokoshin ${ }^{31 b}$, S. Protopopescu ${ }^{24}$, J. Proudfoot ${ }^{5}$, X. Prudent ${ }^{43}$, M. Przybycien ${ }^{37}$, H. Przysiezniak ${ }^{4}$, S. Psoroulas ${ }^{20}$, E. Ptacek ${ }^{114}$, E. Pueschel ${ }^{84}$, J. Purdham ${ }^{87}$, M. Purohit ${ }^{24, z}$, P. Puzo ${ }^{115}$, Y. Pylypchenko ${ }^{62}$, J. Qian ${ }^{87}$, Z. $\operatorname{Qian}^{83}$, Z. Qin ${ }^{41}$, A. Quadt ${ }^{54}$, D.R. Quarrie ${ }^{14}$, W.B. Quayle ${ }^{173}$, F. Quinonez ${ }^{31 a}$, M. $\operatorname{Raas}^{104}$, V. Radescu ${ }^{58 b}$, B. Radics ${ }^{20}$, P. Radloff ${ }^{114}$, T. Rador ${ }^{18 a}$, F. Ragusa ${ }^{89 a, 89 b}$, G. Rahal ${ }^{178}$, A.M. Rahimi ${ }^{109}$, D. Rahm ${ }^{24}$, S. Rajagopalan ${ }^{24}$, M. Rammensee ${ }^{48}$, M. Rammes ${ }^{141}$, A.S. Randle-Conde ${ }^{39}$, K. Randrianarivony ${ }^{28}$, P.N. Ratoff ${ }^{71}$, F. Rauscher ${ }^{98}$, T.C. $\operatorname{Rave}^{48}$, M. Raymond ${ }^{29}$, A.L. Read ${ }^{117}$, D.M. Rebuzzi119a,119b, A. Redelbach ${ }^{174}$, G. Redlinger ${ }^{24}$, R. Reece ${ }^{120}$, K. Reeves ${ }^{40}$, A. Reichold ${ }^{105}$, E. Reinherz-Aronis ${ }^{153}$, A. Reinsch ${ }^{114}$, I. Reisinger ${ }^{42}$, C. Rembser ${ }^{29}$, Z.L. Ren ${ }^{151}$, A. Renaud ${ }^{115}$, P. Renkel ${ }^{39}$, M. Rescigno ${ }^{132 a}$, S. Resconi ${ }^{89 a}$, B. Resende ${ }^{136}$, P. Reznicek $^{98}$, R. Rezvani ${ }^{158}$, A. Richards ${ }^{77}$, R. Richter ${ }^{99}$, E. Richter-Was ${ }^{4, a c}$, M. Ridel ${ }^{78}$, M. Rijpstra ${ }^{105}$, M. Rijssenbeek ${ }^{148}$, A. Rimoldi ${ }^{119 a, 119 b}$, L. Rinaldi ${ }^{19 a}$, R.R. $\operatorname{Rios}^{39}$, I. Riu ${ }^{11}$, G. Rivoltella ${ }^{89 a, 89 b}$, F. Rizatdinova ${ }^{112}$, E. Rizvi ${ }^{75}$, S.H. Robertson ${ }^{85, j}$, A. Robichaud-Veronneau ${ }^{118}$, D. Robinson ${ }^{27}$, J.E.M. Robinson ${ }^{77}$, M. Robinson ${ }^{114}$, A. Robson ${ }^{53}$, J.G. Rocha de Lima ${ }^{106}$, C. Roda ${ }^{122 a, 122 b}$, D. Roda Dos Santos ${ }^{29}$, D. Rodriguez ${ }^{162}$, A. Roe ${ }^{54}$, S. Roe ${ }^{29}$, O. Røhne ${ }^{117}$, V. Rojo ${ }^{1}$, S. Rolli ${ }^{161}$, A. Romaniouk ${ }^{96}$, M. Romano ${ }^{19 a, 19 b}$, V.M. Romanov ${ }^{64}$, G. Romeo ${ }^{26}$, E. Romero Adam ${ }^{167}$, L. Roos ${ }^{78}$, E. Ros ${ }^{167}$, S. Rosati ${ }^{132 a}$, K. Rosbach ${ }^{49}$, A. Rose ${ }^{149}$, M. $\operatorname{Rose}^{76}$, G.A. Rosenbaum ${ }^{158}$, E.I. Rosenberg ${ }^{63}$, P.L. Rosendahl ${ }^{13}$, O. Rosenthal ${ }^{141}$, L. Rosselet ${ }^{49}$, V. Rossetti ${ }^{11}$, E. Rossi ${ }^{132 a, 132 b}$, L.P. Rossi ${ }^{50 a}$, M. Rotaru ${ }^{25 a}$, I. Roth ${ }^{172}$, J. Rothberg ${ }^{138}$, D. Rousseau ${ }^{115}$, C.R. Royon ${ }^{136}$, A. Rozanov ${ }^{83}$, Y. Rozen ${ }^{152}$, X. Ruan ${ }^{32 a, a d}$, I. Rubinskiy ${ }^{41}$, B. Ruckert ${ }^{98}$, N. Ruckstuhl ${ }^{105}$, V.I. Rud ${ }^{97}$, C. Rudolph ${ }^{43}$, G. Rudolph ${ }^{61}$, F. Rühr ${ }^{6}$, F. Ruggieri ${ }^{134 a, 134 b}$, A. Ruiz-Martinez ${ }^{63}$, V. Rumiantsev ${ }^{91, *}$, L. Rumyantsev ${ }^{64}$, K. Runge ${ }^{48}$, Z. Rurikova ${ }^{48}$, N.A. Rusakovich ${ }^{64}$, D.R. Rust ${ }^{60}$, J.P. Rutherfoord ${ }^{6}$, C. Ruwiedel ${ }^{14}$, P. Ruzicka ${ }^{125}$, Y.F. Ryabov ${ }^{121}$, V. Ryadovikov ${ }^{128}$, P. Ryan ${ }^{88}$, M. Rybar ${ }^{126}$, G. Rybkin ${ }^{115}$, N.C. Ryder ${ }^{118}$, S. Rzaeva ${ }^{10}$, A.F. Saavedra ${ }^{150}$, I. Sadeh ${ }^{153}$, H.F-W. Sadrozinski ${ }^{137}$, R. Sadykov ${ }^{64}$, F. Safai Tehrani ${ }^{132 a}$, H. Sakamoto ${ }^{155}$, G. Salamanna ${ }^{75}$, A. Salamon ${ }^{133 a}$, M. Saleem ${ }^{111}$, D. Salihagic ${ }^{99}$, A. Salnikov ${ }^{143}$, J. Salt ${ }^{167}$, B.M. Salvachua Ferrando ${ }^{5}$, D. Salvatore ${ }^{36 a, 36 b}$, F. Salvatore ${ }^{149}$, A. Salvucci ${ }^{104}$, A. Salzburger ${ }^{29}$, D. Sampsonidis ${ }^{154}$, B.H. Samset ${ }^{117}$, A. Sanchez ${ }^{102 a, 102 b}$, V. Sanchez Martinez ${ }^{167}$, H. Sandaker ${ }^{13}$, H.G. Sander ${ }^{81}$, M.P. Sanders ${ }^{98}$, M. Sandhoff ${ }^{175}$, 
T. Sandoval ${ }^{27}$, C. Sandoval ${ }^{162}$, R. Sandstroem ${ }^{99}$, S. Sandvoss ${ }^{175}$, D.P.C. Sankey ${ }^{129}$, A. Sansoni ${ }^{47}$, C. Santamarina Rios ${ }^{85}$, C. Santoni ${ }^{33}$, R. Santonico ${ }^{133 a, 133 b}$, H. Santos ${ }^{124 a}$, J.G. Saraiva ${ }^{124 a}$, T. Sarangi ${ }^{173}$, E. Sarkisyan-Grinbaum ${ }^{7}$, F. Sarri ${ }^{122 a, 122 b}$, G. Sartisohn ${ }^{175}$, O. Sasaki ${ }^{65}$, N. Sasao ${ }^{67}$, I. Satsounkevitch ${ }^{90}$, G. Sauvage ${ }^{4}$, E. Sauvan ${ }^{4}$, J.B. Sauvan ${ }^{115}$, P. Savard ${ }^{158, d}$, V. Savinov ${ }^{123}$, D.O. Savu ${ }^{29}$, L. Sawyer ${ }^{24, l}$, D.H. Saxon ${ }^{53}$, L.P. Says ${ }^{33}$, C. Sbarra ${ }^{19 a}$, A. Sbrizzi19a,19b, O. Scallon ${ }^{93}$, D.A. Scannicchio ${ }^{163}$, M. Scarcella ${ }^{150}$, J. Schaarschmidt ${ }^{115}$, P. Schacht ${ }^{99}$, U. Schäfer ${ }^{81}$, S. Schaepe ${ }^{20}$, S. Schaetzel ${ }^{58 b}$, A.C. Schaffer ${ }^{115}$, D. Schaile ${ }^{98}$, R.D. Schamberger ${ }^{148}$, A.G. Schamov ${ }^{107}$, V. Scharf ${ }^{58 a}$, V.A. Schegelsky ${ }^{121}$, D. Scheirich ${ }^{87}$, M. Schernau ${ }^{163}$, M.I. Scherzer ${ }^{34}$, C. Schiavi ${ }^{50 a, 50 b}$, J. Schieck ${ }^{98}$, M. Schioppa ${ }^{36 a, 36 b}$, S. Schlenker ${ }^{29}$, J.L. Schlereth ${ }^{5}$, E. Schmidt ${ }^{48}$, K. Schmieden ${ }^{20}$, C. Schmitt ${ }^{81}$, S. Schmitt ${ }^{58 b}$, M. Schmitz ${ }^{20}$, A. Schöning ${ }^{58 b}$, M. Schott ${ }^{29}$, D. Schouten ${ }^{159 a}$, J. Schovancova ${ }^{125}$, M. Schram ${ }^{85}$, C. Schroeder ${ }^{81}$, N. Schroer ${ }^{58 c}$, S. Schuh ${ }^{29}$, G. Schuler ${ }^{29}$, M.J. Schultens ${ }^{20}$, J. Schultes ${ }^{175}$, H.-C. Schultz-Coulon ${ }^{58 a}$, H. Schulz ${ }^{15}$, J.W. Schumacher ${ }^{20}$, M. Schumacher ${ }^{48}$, B.A. Schumm ${ }^{137}$, Ph. Schune ${ }^{136}$, C. Schwanenberger ${ }^{82}$, A. Schwartzman ${ }^{143}$, Ph. Schwemling ${ }^{78}$, R. Schwienhorst ${ }^{88}$, R. Schwierz ${ }^{43}$, J. Schwindling ${ }^{136}$, T. Schwindt ${ }^{20}$, M. Schwoerer ${ }^{4}$, W.G. Scott $^{129}$, J. Searcy ${ }^{114}$, G. Sedov $^{41}$, E. Sedykh ${ }^{121}$, E. Segura ${ }^{11}$, S.C. Seidel ${ }^{103}$, A. Seiden ${ }^{137}$, F. Seifert ${ }^{43}$, J.M. Seixas ${ }^{23 a}$, G. Sekhniaidze ${ }^{102 a}$, K.E. Selbach ${ }^{45}$, D.M. Seliverstov ${ }^{121}$, B. Sellden ${ }^{146 a}$, G. Sellers ${ }^{73}$, M. Seman ${ }^{144 b}$, N. Semprini-Cesari ${ }^{19 a, 19 b}$, C. Serfon ${ }^{98}$, L. Serin ${ }^{115}$, L. Serkin $^{54}$, R. Seuster ${ }^{99}$, H. Severini ${ }^{111}$, M.E. Sevior ${ }^{86}$, A. Sfyrla ${ }^{29}$, E. Shabalina ${ }^{54}$, M. Shamim ${ }^{114}$, L.Y. Shan ${ }^{32 a}$, J.T. Shank ${ }^{21}$, Q.T. Shao ${ }^{86}$, M. Shapiro ${ }^{14}$, P.B. Shatalov ${ }^{95}$, L. Shaver ${ }^{6}$, K. Shaw ${ }^{164 a, 164 c}$, D. Sherman ${ }^{176}$, P. Sherwood ${ }^{77}$, A. Shibata ${ }^{108}$, H. Shichi ${ }^{101}$, S. Shimizu ${ }^{29}$, M. Shimojima ${ }^{100}$, T. Shin $^{56}$, M. Shiyakova ${ }^{64}$, A. Shmeleva ${ }^{94}$, M.J. Shochet ${ }^{30}$, D. Short ${ }^{118}$, S. Shrestha ${ }^{63}$, E. Shulga ${ }^{96}$, M.A. Shupe ${ }^{6}$, P. Sicho ${ }^{125}$, A. Sidoti ${ }^{132 a}$, F. Siegert ${ }^{48}$, Dj. Sijacki ${ }^{12 a}$, O. Silbert ${ }^{172}$, J. Silva ${ }^{124 a, b}$, Y. Silver ${ }^{153}$, D. Silverstein ${ }^{143}$, S.B. Silverstein ${ }^{146 a}$, V. Simak ${ }^{127}$, O. Simard ${ }^{136}$, Lj. Simic ${ }^{12 a}$, S. Simion ${ }^{115}$, B. Simmons ${ }^{77}$, M. Simonyan ${ }^{35}$, P. Sinervo ${ }^{158}$, N.B. Sinev ${ }^{114}$, V. Sipica ${ }^{141}$, G. Siragusa ${ }^{174}$, A. Sircar $^{24}$, A.N. Sisakyan ${ }^{64}$, S.Yu. Sivoklokov ${ }^{97}$, J. Sjölin ${ }^{146 a, 146 b}$, T.B. Sjursen ${ }^{13}$, L.A. Skinnari ${ }^{14}$, H.P. Skottowe ${ }^{57}$, K. Skovpen ${ }^{107}$, P. Skubic ${ }^{111}$, N. Skvorodnev ${ }^{22}$, M. Slater ${ }^{17}$, T. Slavicek ${ }^{127}$, K. Sliwa ${ }^{161}$, J. Sloper ${ }^{29}$, V. Smakhtin ${ }^{172}$, B.H. Smart ${ }^{45}$, S.Yu. Smirnov ${ }^{96}$, Y. Smirnov ${ }^{96}$, L.N. Smirnova ${ }^{97}$, O. Smirnova ${ }^{79}$, B.C. Smith ${ }^{57}$, D. Smith ${ }^{143}$, K.M. Smith ${ }^{53}$, M. Smizanska ${ }^{71}$, K. Smolek ${ }^{127}$, A.A. Snesarev ${ }^{94}$, S.W. Snow ${ }^{82}$, J. Snow ${ }^{111}$, J. Snuverink ${ }^{105}$, S. Snyder ${ }^{24}$, M. Soares ${ }^{124 a}$, R. Sobie ${ }^{169, j}$, J. Sodomka ${ }^{127}$, A. Soffer ${ }^{153}$, C.A. Solans ${ }^{167}$, M. Solar ${ }^{127}$, J. Solc ${ }^{127}$, E. Soldatov ${ }^{96}$, U. Soldevila ${ }^{167}$, E. Solfaroli Camillocci ${ }^{132 a, 132 b}$, A.A. Solodkov ${ }^{128}$, O.V. Solovyanov ${ }^{128}$, N. Soni ${ }^{2}$, V. Sopko ${ }^{127}$, B. Sopko ${ }^{127}$, M. Sosebee ${ }^{7}$, R. Soualah ${ }^{164 a, 164 c}$, A. Soukharev ${ }^{107}$, S. Spagnolo ${ }^{72 a, 72 b}$, F. Spanò ${ }^{76}$, R. Spighi ${ }^{19 a}$, G. Spigo ${ }^{29}$, F. Spila ${ }^{132 a, 132 b}$, R. Spiwoks ${ }^{29}$, M. Spousta ${ }^{126}$, T. Spreitzer ${ }^{158}$, B. Spurlock ${ }^{7}$, R.D. St. Denis ${ }^{53}$, J. Stahlman ${ }^{120}$, R. Stamen ${ }^{58 a}$, E. Stanecka ${ }^{38}$, R.W. Stanek ${ }^{5}$, C. Stanescu ${ }^{134 a}$, S. Stapnes ${ }^{117}$, E.A. Starchenko ${ }^{128}$, J. Stark ${ }^{55}$, P. Staroba ${ }^{125}$, P. Starovoitov ${ }^{91}$, A. Staude ${ }^{98}$, P. Stavina ${ }^{144 a}$, G. Stavropoulos ${ }^{14}$, G. Steele ${ }^{53}$, P. Steinbach ${ }^{43}$, P. Steinberg ${ }^{24}$, I. Stekl ${ }^{127}$, B. Stelzer ${ }^{142}$, H.J. Stelzer ${ }^{88}$, O. Stelzer-Chilton ${ }^{159 a}$, H. Stenzel ${ }^{52}$, S. Stern ${ }^{99}$, K. Stevenson ${ }^{75}$, G.A. Stewart ${ }^{29}$, J.A. Stillings ${ }^{20}$, M.C. Stockton ${ }^{85}$, K. Stoerig ${ }^{48}$, G. Stoicea ${ }^{25 a}$, 
S. Stonjek ${ }^{99}$, P. Strachota ${ }^{126}$, A.R. Stradling 7 , A. Straessner ${ }^{43}$, J. Strandberg ${ }^{147}$, S. Strandberg ${ }^{146 a, 146 b}$, A. Strandlie ${ }^{117}$, M. Strang ${ }^{109}$, E. Strauss ${ }^{143}$, M. Strauss ${ }^{111}$, P. Strizenec ${ }^{144 b}$, R. Ströhmer ${ }^{174}$, D.M. Strom ${ }^{114}$, J.A. Strong ${ }^{76, *}$, R. Stroynowski ${ }^{39}$, J. Strube ${ }^{129}$, B. Stugu ${ }^{13}$, I. Stumer ${ }^{24, *}$, J. Stupak ${ }^{148}$, P. Sturm ${ }^{175}$, N.A. Styles ${ }^{41}$, D.A. Soh ${ }^{151, u}$, D. $\mathrm{Su}^{143}$, HS. Subramania ${ }^{2}$, A. Succurro ${ }^{11}$, Y. Sugaya ${ }^{116}$, T. Sugimoto ${ }^{101}$, C. Suhr ${ }^{106}$, K. Suita ${ }^{66}$, M. Suk ${ }^{126}$, V.V. Sulin ${ }^{94}$, S. Sultansoy ${ }^{3 d}$, T. Sumida ${ }^{67}$, X. Sun ${ }^{55}$, J.E. Sundermann ${ }^{48}$, K. Suruliz ${ }^{139}$, S. Sushkov ${ }^{11}$, G. Susinno ${ }^{36 a, 36 b}$, M.R. Sutton ${ }^{149}$, Y. Suzuki ${ }^{65}$, Y. Suzuki ${ }^{66}$, M. Svatos ${ }^{125}$, Yu.M. Sviridov ${ }^{128}$, S. Swedish ${ }^{168}$, I. Sykora ${ }^{144 a}$, T. Sykora ${ }^{126}$, B. Szeless ${ }^{29}$, J. Sánchez ${ }^{167}$, D. Ta ${ }^{105}$,

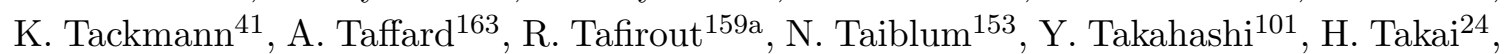
R. Takashima ${ }^{68}$, H. Takeda ${ }^{66}$, T. Takeshita ${ }^{140}$, Y. Takubo ${ }^{65}$, M. Talby ${ }^{83}$, A. Talyshev $^{107, f}$, M.C. Tamsett ${ }^{24}$, J. Tanaka ${ }^{155}$, R. Tanaka ${ }^{115}$, S. Tanaka ${ }^{131}$, S. Tanaka ${ }^{65}$, Y. Tanaka ${ }^{100}$, A.J. Tanasijczuk ${ }^{142}$, K. Tani ${ }^{66}$, N. Tannoury ${ }^{83}$, G.P. Tappern ${ }^{29}$, S. Tapprogge ${ }^{81}$, D. Tardif ${ }^{158}$, S. Tarem ${ }^{152}$, F. Tarrade ${ }^{28}$, G.F. Tartarelli ${ }^{89 a}$, P. Tas $^{126}$, M. Tasevsky ${ }^{125}$, E. Tassi ${ }^{36 a, 36 b}$, M. Tatarkhanov ${ }^{14}$, Y. Tayalati ${ }^{135 d}$, C. Taylor ${ }^{77}$, F.E. Taylor ${ }^{92}$, G.N. Taylor ${ }^{86}$, W. Taylor ${ }^{159 b}$, M. Teinturier ${ }^{115}$, M. Teixeira Dias Castanheira ${ }^{75}$, P. Teixeira-Dias ${ }^{76}$, K.K. Temming ${ }^{48}$, H. Ten Kate ${ }^{29}$, P.K. Teng ${ }^{151}$, S. Terada ${ }^{65}$, K. Terashi ${ }^{155}$, J. Terron ${ }^{80}$, M. Testa ${ }^{47}$, R.J. Teuscher ${ }^{158, j}$, J. Thadome ${ }^{175}$, J. Therhaag ${ }^{20}$, T. Theveneaux-Pelzer ${ }^{78}$, M. Thioye ${ }^{176}$, S. Thoma ${ }^{48}$, J.P. Thomas ${ }^{17}$, E.N. Thompson ${ }^{34}$, P.D. Thompson ${ }^{17}$, P.D. Thompson ${ }^{158}$, A.S. Thompson ${ }^{53}$, L.A. Thomsen ${ }^{35}$, E. Thomson ${ }^{120}$, M. Thomson ${ }^{27}$, R.P. Thun ${ }^{87}$, F. Tian $^{34}$, M.J. Tibbetts ${ }^{14}$, T. Tic ${ }^{125}$, V.O. Tikhomirov ${ }^{94}$, Y.A. Tikhonov ${ }^{107, f}$, S. Timoshenko ${ }^{96}$, P. Tipton ${ }^{176}$, F.J. Tique Aires Viegas ${ }^{29}$,

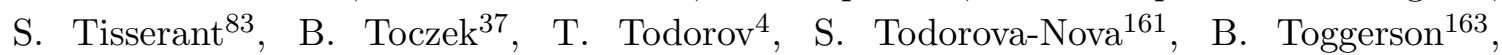
J. Tojo ${ }^{65}$, S. Tokár ${ }^{144 a}$, K. Tokunaga ${ }^{66}$, K. Tokushuku ${ }^{65}$, K. Tollefson ${ }^{88}$, M. Tomoto ${ }^{101}$, L. Tompkins ${ }^{30}$, K. Toms ${ }^{103}$, G. Tong ${ }^{32 a}$, A. Tonoyan ${ }^{13}$, C. Topfel ${ }^{16}$, N.D. Topilin ${ }^{64}$,

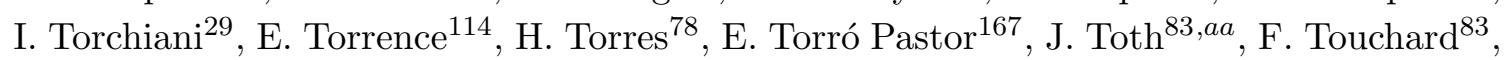
D.R. Tovey ${ }^{139}$, T. Trefzger ${ }^{174}$, L. Tremblet ${ }^{29}$, A. Tricoli ${ }^{29}$, I.M. Trigger ${ }^{159 a}$, S. Trincaz-

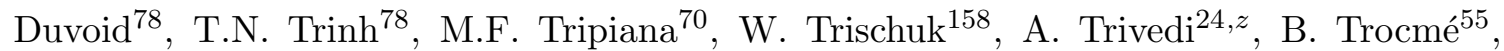

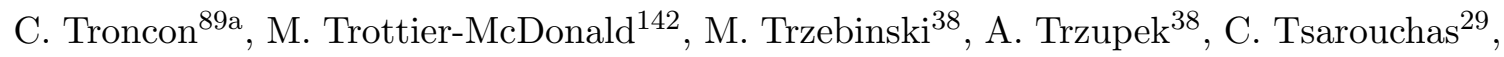
J.C-L. Tseng ${ }^{118}$, M. Tsiakiris ${ }^{105}$, P.V. Tsiareshka ${ }^{90}$, D. Tsionou ${ }^{4, a e}$, G. Tsipolitis ${ }^{9}$, V. Tsiskaridze ${ }^{48}$, E.G. Tskhadadze ${ }^{51 a}$, I.I. Tsukerman ${ }^{95}$, V. Tsulaia ${ }^{14}$, J.-W. Tsung ${ }^{20}$, S. Tsuno ${ }^{65}$, D. Tsybychev ${ }^{148}$, A. Tua ${ }^{139}$, A. Tudorache $^{25 a}$, V. Tudorache ${ }^{25 a}$, J.M. Tuggle $^{30}$, M. Turala ${ }^{38}$, D. Turecek ${ }^{127}$, I. Turk Cakir ${ }^{3 e}$, E. Turlay ${ }^{105}$, R. Turra ${ }^{89 a, 89 b}$, P.M. Tuts ${ }^{34}$,

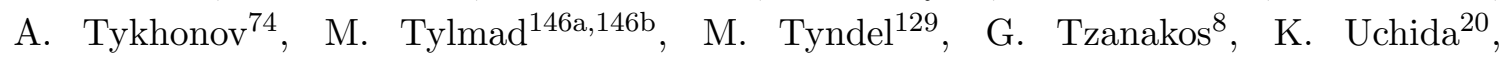
I. Ueda ${ }^{155}$, R. Ueno ${ }^{28}$, M. Ugland ${ }^{13}$, M. Uhlenbrock ${ }^{20}$, M. Uhrmacher ${ }^{54}$, F. Ukegawa ${ }^{160}$,

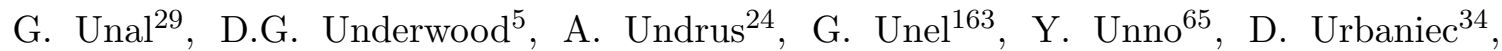
G. Usai ${ }^{7}$, M. Uslenghi ${ }^{119 a, 119 b}$, L. Vacavant ${ }^{83}$, V. Vacek ${ }^{127}$, B. Vachon ${ }^{85}$, S. Vahsen ${ }^{14}$, J. Valenta ${ }^{125}$, P. Valente ${ }^{132 a}$, S. Valentinetti19a,19b ${ }^{19}$ S. Valkar ${ }^{126}$, E. Valladolid Gallego ${ }^{167}$, S. Vallecorsa ${ }^{152}$, J.A. Valls Ferrer ${ }^{167}$, H. van der Graaf ${ }^{105}$, E. van der Kraaij ${ }^{105}$, R. Van Der Leeuw ${ }^{105}$, E. van der Poel $^{105}$, D. van der $\operatorname{Ster}^{29}$, N. van Eldik ${ }^{84}$, P. van Gemmeren ${ }^{5}$, Z. van Kesteren ${ }^{105}$, I. van Vulpen ${ }^{105}$, M. Vanadia ${ }^{99}$, W. Vandelli ${ }^{29}$, G. Vandoni ${ }^{29}$, A. Vaniachine ${ }^{5}$, P. Vankov ${ }^{41}$, F. Vannucci ${ }^{78}$, F. Varela Rodriguez ${ }^{29}$, R. Vari ${ }^{132 a}$, E.W. Varnes $^{6}$, D. Varouchas ${ }^{14}$, A. Vartapetian ${ }^{7}$, K.E. Varvell ${ }^{150}$, 
V.I. Vassilakopoulos ${ }^{56}$, F. Vazeille ${ }^{33}$, G. Vegni ${ }^{89 a, 89 b}$, J.J. Veillet ${ }^{115}$, C. Vellidis ${ }^{8}$,

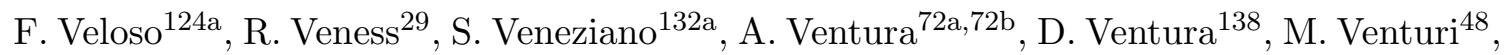
N. Venturi ${ }^{158}$, V. Vercesi ${ }^{119 a}$, M. Verducci ${ }^{138}$, W. Verkerke ${ }^{105}$, J.C. Vermeulen ${ }^{105}$, A. Vest ${ }^{43}$, M.C. Vetterli ${ }^{142, d}$, I. Vichou ${ }^{165}$, T. Vickey ${ }^{145 b, a f}$, O.E. Vickey Boeriu ${ }^{145 b}$, G.H.A. Viehhauser ${ }^{118}$, S. Viel ${ }^{168}$, M. Villa ${ }^{19 a, 19 b}$, M. Villaplana Perez ${ }^{167}$, E. Vilucchi ${ }^{47}$, M.G. Vincter ${ }^{28}$, E. $\operatorname{Vinek}^{29}$, V.B. Vinogradov ${ }^{64}$, M. Virchaux ${ }^{136, *}$, J. Virzi ${ }^{14}$, O. Vitells ${ }^{172}$, M. Viti ${ }^{41}$, I. Vivarelli ${ }^{48}$, F. Vives Vaque $^{2}$, S. Vlachos ${ }^{9}$, D. Vladoiu ${ }^{98}$,

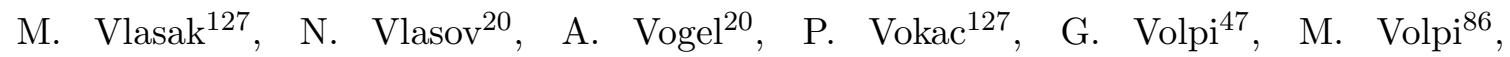
G. Volpini ${ }^{89 a}$, H. von der Schmitt $^{99}$, J. von Loeben $^{99}$, H. von Radziewski ${ }^{48}$, E. von Toerne ${ }^{20}$, V. Vorobel ${ }^{126}$, A.P. Vorobiev ${ }^{128}$, V. Vorwerk ${ }^{11}$, M. Vos ${ }^{167}$, R. $\operatorname{Voss}^{29}$, T.T. Voss ${ }^{175}$, J.H. Vossebeld ${ }^{73}$, N. Vranjes ${ }^{136}$, M. Vranjes Milosavljevic ${ }^{105}$, V. Vrba ${ }^{125}$, M. Vreeswijk ${ }^{105}$, T. Vu Anh ${ }^{48}$, R. Vuillermet ${ }^{29}$, I. Vukotic ${ }^{115}$, W. Wagner ${ }^{175}$, P. Wagner ${ }^{120}$, H. Wahlen ${ }^{175}$, J. Wakabayashi ${ }^{101}$, J. Walbersloh ${ }^{42}$, S. Walch ${ }^{87}$, J. Walder ${ }^{71}$, R. Walker ${ }^{98}$, W. Walkowiak ${ }^{141}$, R. Wall ${ }^{176}$, P. Waller ${ }^{73}$, C. Wang ${ }^{44}$, H. Wang ${ }^{173}$, H. Wang ${ }^{32 b, a g}$, J. Wang ${ }^{151}$, J. Wang ${ }^{55}$, J.C. Wang ${ }^{138}$, R. Wang ${ }^{103}$, S.M. Wang ${ }^{151}$, A. Warburton ${ }^{85}$, C.P. Ward ${ }^{27}$, M. Warsinsky ${ }^{48}$, P.M. Watkins ${ }^{17}$, A.T. Watson ${ }^{17}$, I.J. Watson ${ }^{150}$, M.F. Watson ${ }^{17}$, G. Watts ${ }^{138}$, S. Watts ${ }^{82}$, A.T. Waugh ${ }^{150}$, B.M. Waugh ${ }^{77}$, M. Weber ${ }^{129}$, M.S. Weber ${ }^{16}$, P. Weber ${ }^{54}$, A.R. Weidberg ${ }^{118}$, P. Weigell ${ }^{99}$, J. Weingarten ${ }^{54}$, C. Weiser ${ }^{48}$, H. Wellenstein ${ }^{22}$, P.S. Wells ${ }^{29}$, M. Wen ${ }^{47}$, T. Wenaus ${ }^{24}$, S. Wendler ${ }^{123}$, Z. Weng ${ }^{151, u}$, T. Wengler ${ }^{29}$, S. Wenig ${ }^{29}$, N. Wermes ${ }^{20}$, M. Werner ${ }^{48}$, P. Werner ${ }^{29}$, M. Werth ${ }^{163}$, M. Wessels ${ }^{58 a}$, C. Weydert ${ }^{55}$, K. Whalen ${ }^{28}$, S.J. Wheeler-Ellis ${ }^{163}$, S.P. Whitaker ${ }^{21}$, A. White ${ }^{7}$, M.J. White ${ }^{86}$, S.R. Whitehead ${ }^{118}$, D. Whiteson ${ }^{163}$, D. Whittington ${ }^{60}$, F. Wicek ${ }^{115}$, D. Wicke ${ }^{175}$, F.J. Wickens ${ }^{129}$, W. Wiedenmann ${ }^{173}$, M. Wielers ${ }^{129}$, P. Wienemann ${ }^{20}$, C. Wiglesworth ${ }^{75}$, L.A.M. Wiik-Fuchs ${ }^{48}$, P.A. Wijeratne ${ }^{77}$, A. Wildauer ${ }^{167}$, M.A. Wildt ${ }^{41, q}$, I. Wilhelm ${ }^{126}$, H.G. Wilkens ${ }^{29}$, J.Z. Will ${ }^{98}$, E. Williams ${ }^{34}$, H.H. Williams ${ }^{120}$, W. Willis ${ }^{34}$, S. Willocq ${ }^{84}$, J.A. Wilson ${ }^{17}$, M.G. Wilson ${ }^{143}$, A. Wilson ${ }^{87}$, I. Wingerter-Seez ${ }^{4}$, S. Winkelmann ${ }^{48}$, F. Winklmeier ${ }^{29}$, M. Wittgen ${ }^{143}$, M.W. Wolter ${ }^{38}$, H. Wolters ${ }^{124 a, h}$, W.C. Wong ${ }^{40}$, G. Wooden ${ }^{87}$, B.K. Wosiek ${ }^{38}$, J. Wotschack ${ }^{29}$, M.J. Woudstra ${ }^{84}$, K.W. Wozniak ${ }^{38}$, K. Wraight ${ }^{53}$, C. Wright ${ }^{53}$, M. Wright ${ }^{53}$, B. Wrona ${ }^{73}$, S.L. $\mathrm{Wu}^{173}, \mathrm{X} . \mathrm{Wu}^{49}, \mathrm{Y} . \mathrm{Wu}^{32 \mathrm{~b}, a h}$, E. Wulf ${ }^{34}$, R. Wunstorf ${ }^{42}$, B.M. Wynne ${ }^{45}$, S. Xella ${ }^{35}$, M. $\mathrm{Xiao}^{136}$, S. $\mathrm{Xie}^{48}, \mathrm{Y} \cdot \mathrm{Xie}^{32 \mathrm{a}}$, C. $\mathrm{Xu}^{32 \mathrm{~b}, w}, \mathrm{D} \cdot \mathrm{Xu}^{139}$, G. $\mathrm{Xu}^{32 \mathrm{a}}$, B. Yabsley ${ }^{150}$, S. Yacoob ${ }^{145 b}$, M. Yamada ${ }^{65}$, H. Yamaguchi ${ }^{155}$, A. Yamamoto ${ }^{65}$, K. Yamamoto ${ }^{63}$, S. Yamamoto ${ }^{155}$, T. Yamamura ${ }^{155}$, T. Yamanaka ${ }^{155}$, J. Yamaoka ${ }^{44}$, T. Yamazaki ${ }^{155}$,

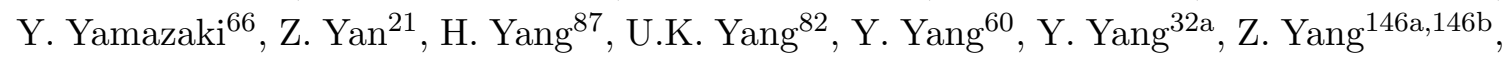

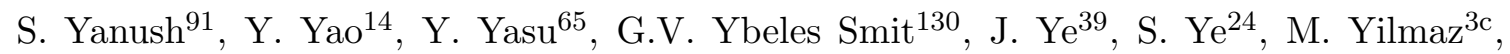
R. Yoosoofmiya ${ }^{123}$, K. Yorita ${ }^{171}$, R. Yoshida ${ }^{5}$, C. Young ${ }^{143}$, S. Youssef ${ }^{21}$, D. Yu ${ }^{24}$, J. Yu $^{7}$, J. Yu ${ }^{112}$, L. Yuan ${ }^{32 \mathrm{a}, a i}$, A. Yurkewicz ${ }^{106}$, B. Zabinski ${ }^{38}$, V.G. Zaets ${ }^{128}$, R. Zaidan ${ }^{62}$, A.M. Zaitsev ${ }^{128}$, Z. Zajacova ${ }^{29}$, L. Zanello132a,132b, P. Zarzhitsky ${ }^{39}$, A. Zaytsev ${ }^{107}$, C. Zeitnitz ${ }^{175}$, M. Zeller ${ }^{176}$, M. Zeman ${ }^{125}$, A. Zemla ${ }^{38}$, C. Zendler ${ }^{20}$, O. Zenin ${ }^{128}$, T. Ženišs ${ }^{144 a}$, Z. Zinonos ${ }^{122 a, 122 b}$, S. Zenz ${ }^{14}$, D. Zerwas ${ }^{115}$, G. Zevi della Porta ${ }^{57}$, Z. Zhan ${ }^{32 d}$, D. Zhang ${ }^{32 \mathrm{~b}, a g}$, H. Zhang ${ }^{88}$, J. Zhang ${ }^{5}$, X. Zhang ${ }^{32 d}$, Z. Zhang ${ }^{115}$, L. Zhao ${ }^{108}$, T. Zhao ${ }^{138}$, Z. Zhao ${ }^{32 b}$, A. Zhemchugov ${ }^{64}$, S. Zheng ${ }^{32 \mathrm{a}}$, J. Zhong ${ }^{118}$, B. Zhou ${ }^{87}$, N. Zhou ${ }^{163}$, Y. Zhou ${ }^{151}$, C.G. Zhu ${ }^{32 \mathrm{~d}}$, H. Zhu ${ }^{41}$, J. Zhu ${ }^{87}$, Y. Zhu ${ }^{32 \mathrm{~b}}$, X. Zhuang ${ }^{98}$, V. Zhuravlov ${ }^{99}$, D. Zieminska ${ }^{60}$, 
R. Zimmermann ${ }^{20}$, S. Zimmermann ${ }^{20}$, S. Zimmermann ${ }^{48}$, M. Ziolkowski ${ }^{141}$, R. Zitoun ${ }^{4}$, L. Živković ${ }^{34}$, V.V. Zmouchko ${ }^{128, *}$, G. Zobernig ${ }^{173}$, A. Zoccoli ${ }^{19 a, 19 b}$, Y. Zolnierowski ${ }^{4}$, A. Zsenei ${ }^{29}$, M. zur Nedden ${ }^{15}$, V. Zutshi ${ }^{106}$, L. Zwalinski ${ }^{29}$ and T. Ohsugi ${ }^{179}$.

1: University at Albany, Albany NY, United States of America

2: Department of Physics, University of Alberta, Edmonton AB, Canada

${ }^{3}$ : ${ }^{(a)}$ Department of Physics, Ankara University, Ankara; ${ }^{(b)}$ Department of Physics, Dumlupinar University, Kutahya; ${ }^{(c)}$ Department of Physics, Gazi University, Ankara; (d) Division of Physics, TOBB University of Economics and Technology, Ankara; ${ }^{(e)}$ Turkish Atomic Energy Authority, Ankara, Turkey

4: LAPP, CNRS/IN2P3 and Université de Savoie, Annecy-le-Vieux, France

5: High Energy Physics Division, Argonne National Laboratory, Argonne IL, United States of America

6: Department of Physics, University of Arizona, Tucson AZ, United States of America

7: Department of Physics, The University of Texas at Arlington, Arlington TX, United States of America

8: Physics Department, University of Athens, Athens, Greece

9: Physics Department, National Technical University of Athens, Zografou, Greece

10: Institute of Physics, Azerbaijan Academy of Sciences, Baku, Azerbaijan

11: Institut de Física d'Altes Energies and Departament de Física de la Universitat Autònoma de Barcelona and ICREA, Barcelona, Spain

12: ${ }^{(a)}$ Institute of Physics, University of Belgrade, Belgrade; ${ }^{(b)}$ Vinca Institute of Nuclear Sciences, University of Belgrade, Belgrade, Serbia

13: Department for Physics and Technology, University of Bergen, Bergen, Norway

14: Physics Division, Lawrence Berkeley National Laboratory and University of California, Berkeley CA, United States of America

15: Department of Physics, Humboldt University, Berlin, Germany

16: Albert Einstein Center for Fundamental Physics and Laboratory for High Energy Physics, University of Bern, Bern, Switzerland

17: School of Physics and Astronomy, University of Birmingham, Birmingham, United Kingdom

18: ${ }^{(a)}$ Department of Physics, Bogazici University, Istanbul; ${ }^{(b)}$ Division of Physics, Dogus University, Istanbul; ${ }^{(c)}$ Department of Physics Engineering, Gaziantep University, Gaziantep; ${ }^{(d)}$ Department of Physics, Istanbul Technical University, Istanbul, Turkey

19: ${ }^{(a)}$ INFN Sezione di Bologna; ${ }^{(b)}$ Dipartimento di Fisica, Università di Bologna, Bologna, Italy

20: Physikalisches Institut, University of Bonn, Bonn, Germany

21: Department of Physics, Boston University, Boston MA, United States of America

22: Department of Physics, Brandeis University, Waltham MA, United States of America

23: ${ }^{(a)}$ Universidade Federal do Rio De Janeiro COPPE/EE/IF, Rio de Janeiro; ${ }^{(b)}$ Federal University of Juiz de Fora (UFJF), Juiz de Fora; ${ }^{(c)}$ Federal University of Sao Joao 
del Rei (UFSJ), Sao Joao del Rei; ${ }^{(d)}$ Instituto de Fisica, Universidade de Sao Paulo, Sao Paulo, Brazil

24: Physics Department, Brookhaven National Laboratory, Upton NY, United States of America

25: ${ }^{(a)}$ National Institute of Physics and Nuclear Engineering, Bucharest; ${ }^{(b)}$ University Politehnica Bucharest, Bucharest; ${ }^{(c)}$ West University in Timisoara, Timisoara, Romania

26: Departamento de Física, Universidad de Buenos Aires, Buenos Aires, Argentina

27: Cavendish Laboratory, University of Cambridge, Cambridge, United Kingdom

28: Department of Physics, Carleton University, Ottawa ON, Canada

29: CERN, Geneva, Switzerland

30: Enrico Fermi Institute, University of Chicago, Chicago IL, United States of America

31: ${ }^{(a)}$ Departamento de Fisica, Pontificia Universidad Católica de Chile, Santiago;

${ }^{(b)}$ Departamento de Física, Universidad Técnica Federico Santa María, Valparaíso, Chile

32: ${ }^{(a)}$ Institute of High Energy Physics, Chinese Academy of Sciences, Beijing; ${ }^{(b)}$ Department of Modern Physics, University of Science and Technology of China, Anhui; ${ }^{(c)}$ Department of Physics, Nanjing University, Jiangsu; ${ }^{(d)}$ School of Physics, Shandong University, Shandong, China

33: Laboratoire de Physique Corpusculaire, Clermont Université and Université Blaise Pascal and CNRS/IN2P3, Aubiere Cedex, France

34: Nevis Laboratory, Columbia University, Irvington NY, United States of America

35: Niels Bohr Institute, University of Copenhagen, Kobenhavn, Denmark

36: ${ }^{(a)}$ INFN Gruppo Collegato di Cosenza; ${ }^{(b)}$ Dipartimento di Fisica, Università della Calabria, Arcavata di Rende, Italy

37: AGH University of Science and Technology, Faculty of Physics and Applied Computer Science, Krakow, Poland

38: The Henryk Niewodniczanski Institute of Nuclear Physics, Polish Academy of Sciences, Krakow, Poland

39: Physics Department, Southern Methodist University, Dallas TX, United States of America

40: Physics Department, University of Texas at Dallas, Richardson TX, United States of America

41: DESY, Hamburg and Zeuthen, Germany

42: Institut für Experimentelle Physik IV, Technische Universität Dortmund, Dortmund, Germany

43: Institut für Kern- und Teilchenphysik, Technical University Dresden, Dresden, Germany

44: Department of Physics, Duke University, Durham NC, United States of America

45: SUPA - School of Physics and Astronomy, University of Edinburgh, Edinburgh, United Kingdom

46: Fachhochschule Wiener Neustadt, Johannes Gutenbergstrasse 32700 Wiener Neustadt, Austria 
47: INFN Laboratori Nazionali di Frascati, Frascati, Italy

48: Fakultät für Mathematik und Physik, Albert-Ludwigs-Universität, Freiburg i.Br., Germany

49: Section de Physique, Université de Genève, Geneva, Switzerland

${ }^{50}$ : ${ }^{(a)}$ INFN Sezione di Genova; ${ }^{(b)}$ Dipartimento di Fisica, Università di Genova, Genova, Italy

51: ${ }^{(a)}$ E.Andronikashvili Institute of Physics, Tbilisi State University, Tbilisi; ${ }^{(b)}$ High Energy Physics Institute, Tbilisi State University, Tbilisi, Georgia

52: II Physikalisches Institut, Justus-Liebig-Universität Giessen, Giessen, Germany

53: SUPA - School of Physics and Astronomy, University of Glasgow, Glasgow, United Kingdom

54: II Physikalisches Institut, Georg-August-Universität, Göttingen, Germany

55: Laboratoire de Physique Subatomique et de Cosmologie, Université Joseph Fourier and CNRS/IN2P3 and Institut National Polytechnique de Grenoble, Grenoble, France

56: Department of Physics, Hampton University, Hampton VA, United States of America

57: Laboratory for Particle Physics and Cosmology, Harvard University, Cambridge MA, United States of America

58: (a) Kirchhoff-Institut für Physik, Ruprecht-Karls-Universität Heidelberg, Heidelberg; ${ }^{(b)}$ Physikalisches Institut, Ruprecht-Karls-Universität Heidelberg, Heidelberg; (c)ZITI Institut für technische Informatik, Ruprecht-Karls-Universität Heidelberg, Mannheim, Germany

59: Faculty of Applied Information Science, Hiroshima Institute of Technology, Hiroshima, Japan

60: Department of Physics, Indiana University, Bloomington IN, United States of America

61: Institut für Astro- und Teilchenphysik, Leopold-Franzens-Universität, Innsbruck, Austria

62: University of Iowa, Iowa City IA, United States of America

63: Department of Physics and Astronomy, Iowa State University, Ames IA, United States of America

64: Joint Institute for Nuclear Research, JINR Dubna, Dubna, Russia

65: KEK, High Energy Accelerator Research Organization, Tsukuba, Japan

66: Graduate School of Science, Kobe University, Kobe, Japan

67: Faculty of Science, Kyoto University, Kyoto, Japan

68: Kyoto University of Education, Kyoto, Japan

70: Instituto de Física La Plata, Universidad Nacional de La Plata and CONICET, La Plata, Argentina

71: Physics Department, Lancaster University, Lancaster, United Kingdom

72: ${ }^{(a)}$ INFN Sezione di Lecce; ${ }^{(b)}$ Dipartimento di Fisica, Università del Salento, Lecce, Italy

73: Oliver Lodge Laboratory, University of Liverpool, Liverpool, United Kingdom

74: Department of Physics, Jožef Stefan Institute and University of Ljubljana, Ljubljana, Slovenia 
75: School of Physics and Astronomy, Queen Mary University of London, London, United Kingdom

76: Department of Physics, Royal Holloway University of London, Surrey, United Kingdom

77: Department of Physics and Astronomy, University College London, London, United Kingdom

78: Laboratoire de Physique Nucléaire et de Hautes Energies, UPMC and Université Paris-Diderot and CNRS/IN2P3, Paris, France

79: Fysiska institutionen, Lunds universitet, Lund, Sweden

80: Departamento de Fisica Teorica C-15, Universidad Autonoma de Madrid, Madrid, Spain

81: Institut für Physik, Universität Mainz, Mainz, Germany

82: School of Physics and Astronomy, University of Manchester, Manchester, United Kingdom

83: CPPM, Aix-Marseille Université and CNRS/IN2P3, Marseille, France

84: Department of Physics, University of Massachusetts, Amherst MA, United States of America

85: Department of Physics, McGill University, Montreal QC, Canada

86: School of Physics, University of Melbourne, Victoria, Australia

87: Department of Physics, The University of Michigan, Ann Arbor MI, United States of America

88: Department of Physics and Astronomy, Michigan State University, East Lansing MI, United States of America

89: ${ }^{(a)}$ INFN Sezione di Milano; ${ }^{(b)}$ Dipartimento di Fisica, Università di Milano, Milano, Italy

90: B.I. Stepanov Institute of Physics, National Academy of Sciences of Belarus, Minsk, Republic of Belarus

91: National Scientific and Educational Centre for Particle and High Energy Physics, Minsk, Republic of Belarus

92: Department of Physics, Massachusetts Institute of Technology, Cambridge MA, United States of America

93: Group of Particle Physics, University of Montreal, Montreal QC, Canada

94: P.N. Lebedev Institute of Physics, Academy of Sciences, Moscow, Russia

95: Institute for Theoretical and Experimental Physics (ITEP), Moscow, Russia

96: Moscow Engineering and Physics Institute (MEPhI), Moscow, Russia

97: Skobeltsyn Institute of Nuclear Physics, Lomonosov Moscow State University, Moscow, Russia

98: Fakultät für Physik, Ludwig-Maximilians-Universität München, München, Germany

99: Max-Planck-Institut für Physik (Werner-Heisenberg-Institut), München, Germany

100: Nagasaki Institute of Applied Science, Nagasaki, Japan

101: Graduate School of Science, Nagoya University, Nagoya, Japan

102: ${ }^{(a)}$ INFN Sezione di Napoli; ${ }^{(b)}$ Dipartimento di Scienze Fisiche, Università di Napoli, Napoli, Italy 
103: Department of Physics and Astronomy, University of New Mexico, Albuquerque NM, United States of America

104: Institute for Mathematics, Astrophysics and Particle Physics, Radboud University Nijmegen/Nikhef, Nijmegen, Netherlands

105. Nikhef National Institute for Subatomic Physics and University of Amsterdam, Amsterdam, Netherlands

106: Department of Physics, Northern Illinois University, DeKalb IL, United States of America

107: Budker Institute of Nuclear Physics, SB RAS, Novosibirsk, Russia

108: Department of Physics, New York University, New York NY, United States of America

109: Ohio State University, Columbus OH, United States of America

110: Faculty of Science, Okayama University, Okayama, Japan

111: Homer L. Dodge Department of Physics and Astronomy, University of Oklahoma, Norman OK, United States of America

112: Department of Physics, Oklahoma State University, Stillwater OK, United States of America

113: Palacký University, RCPTM, Olomouc, Czech Republic

114: Center for High Energy Physics, University of Oregon, Eugene OR, United States of America

115: LAL, Univ. Paris-Sud and CNRS/IN2P3, Orsay, France

116: Graduate School of Science, Osaka University, Osaka, Japan

117: Department of Physics, University of Oslo, Oslo, Norway

118: Department of Physics, Oxford University, Oxford, United Kingdom

119: ${ }^{(a)}$ INFN Sezione di Pavia; ${ }^{(b)}$ Dipartimento di Fisica, Università di Pavia, Pavia, Italy

120: Department of Physics, University of Pennsylvania, Philadelphia PA, United States of America

121: Petersburg Nuclear Physics Institute, Gatchina, Russia

122: ${ }^{(a)}$ INFN Sezione di Pisa; ${ }^{(b)}$ Dipartimento di Fisica E. Fermi, Università di Pisa, Pisa, Italy

123: Department of Physics and Astronomy, University of Pittsburgh, Pittsburgh PA, United States of America

124: ${ }^{(a)}$ Laboratorio de Instrumentacao e Fisica Experimental de Particulas - LIP, Lisboa, Portugal; ${ }^{(b)}$ Departamento de Fisica Teorica y del Cosmos and CAFPE, Universidad de Granada, Granada, Spain

125: Institute of Physics, Academy of Sciences of the Czech Republic, Praha, Czech Republic

126: Faculty of Mathematics and Physics, Charles University in Prague, Praha, Czech Republic

127: Czech Technical University in Prague, Praha, Czech Republic

128: State Research Center Institute for High Energy Physics, Protvino, Russia

129: Particle Physics Department, Rutherford Appleton Laboratory, Didcot, United Kingdom 
130: Physics Department, University of Regina, Regina SK, Canada

131: Ritsumeikan University, Kusatsu, Shiga, Japan

132: ${ }^{(a)}$ INFN Sezione di Roma I; ${ }^{\left({ }^{b}\right)}$ Dipartimento di Fisica, Università La Sapienza, Roma, Italy

133: ${ }^{(a)}$ INFN Sezione di Roma Tor Vergata; ${ }^{(b)}$ Dipartimento di Fisica, Università di Roma Tor Vergata, Roma, Italy

134: ${ }^{(a)}$ INFN Sezione di Roma Tre; ${ }^{(b)}$ Dipartimento di Fisica, Università Roma Tre, Roma, Italy

135: ${ }^{(a)}$ Faculté des Sciences Ain Chock, Réseau Universitaire de Physique des Hautes Energies - Université Hassan II, Casablanca; ${ }^{(b)}$ Centre National de l'Energie des Sciences Techniques Nucleaires, Rabat; ${ }^{(c)}$ Faculté des Sciences Semlalia, Université Cadi Ayyad, LPHEA-Marrakech; ${ }^{(d)}$ Faculté des Sciences, Université Mohamed Premier and LPTPM, Oujda; ${ }^{(e)}$ Faculté des Sciences, Université Mohammed V- Agdal, Rabat, Morocco

136: DSM/IRFU (Institut de Recherches sur les Lois Fondamentales de l'Univers), CEA Saclay (Commissariat a l'Energie Atomique), Gif-sur-Yvette, France

137: Santa Cruz Institute for Particle Physics, University of California Santa Cruz, Santa Cruz CA, United States of America

138: Department of Physics, University of Washington, Seattle WA, United States of America

139: Department of Physics and Astronomy, University of Sheffield, Sheffield, United Kingdom

140: Department of Physics, Shinshu University, Nagano, Japan

141: Fachbereich Physik, Universität Siegen, Siegen, Germany

142: Department of Physics, Simon Fraser University, Burnaby BC, Canada

143: SLAC National Accelerator Laboratory, Stanford CA, United States of America

144: (a) Faculty of Mathematics, Physics \& Informatics, Comenius University, Bratislava;

(b) Department of Subnuclear Physics, Institute of Experimental Physics of the Slovak Academy of Sciences, Kosice, Slovak Republic

145: ${ }^{(a)}$ Department of Physics, University of Johannesburg, Johannesburg; ${ }^{(b)}$ School of Physics, University of the Witwatersrand, Johannesburg, South Africa

146: ${ }^{(a)}$ Department of Physics, Stockholm University; ${ }^{(b)}$ The Oskar Klein Centre, Stockholm, Sweden

147: Physics Department, Royal Institute of Technology, Stockholm, Sweden

148: Departments of Physics \& Astronomy and Chemistry, Stony Brook University, Stony Brook NY, United States of America

149: Department of Physics and Astronomy, University of Sussex, Brighton, United Kingdom

150: School of Physics, University of Sydney, Sydney, Australia

151: Institute of Physics, Academia Sinica, Taipei, Taiwan

152: Department of Physics, Technion: Israel Inst. of Technology, Haifa, Israel

153: Raymond and Beverly Sackler School of Physics and Astronomy, Tel Aviv University, Tel Aviv, Israel 
154: Department of Physics, Aristotle University of Thessaloniki, Thessaloniki, Greece

155: International Center for Elementary Particle Physics and Department of Physics, The University of Tokyo, Tokyo, Japan

156: Graduate School of Science and Technology, Tokyo Metropolitan University, Tokyo, Japan

157: Department of Physics, Tokyo Institute of Technology, Tokyo, Japan

158: Department of Physics, University of Toronto, Toronto ON, Canada

159: ${ }^{(a)}$ TRIUMF, Vancouver BC; ${ }^{(b)}$ Department of Physics and Astronomy, York University, Toronto ON, Canada

160: Institute of Pure and Applied Sciences, University of Tsukuba,1-1-1 Tennodai,Tsukuba, Ibaraki 305-8571, Japan

161: Science and Technology Center, Tufts University, Medford MA, United States of America

162: Centro de Investigaciones, Universidad Antonio Narino, Bogota, Colombia

163: Department of Physics and Astronomy, University of California Irvine, Irvine CA, United States of America

164: ${ }^{(a)}$ INFN Gruppo Collegato di Udine; ${ }^{(b)}$ ICTP, Trieste; ${ }^{(c)}$ Dipartimento di Chimica, Fisica e Ambiente, Università di Udine, Udine, Italy

165: Department of Physics, University of Illinois, Urbana IL, United States of America

166: Department of Physics and Astronomy, University of Uppsala, Uppsala, Sweden

167: Instituto de Física Corpuscular (IFIC) and Departamento de Física Atómica, Molecular y Nuclear and Departamento de Ingeniería Electrónica and Instituto de Microelectrónica de Barcelona (IMB-CNM), University of Valencia and CSIC, Valencia, Spain

168: Department of Physics, University of British Columbia, Vancouver BC, Canada

169: Department of Physics and Astronomy, University of Victoria, Victoria BC, Canada

171: Waseda University, Tokyo, Japan

172: Department of Particle Physics, The Weizmann Institute of Science, Rehovot, Israel

173: Department of Physics, University of Wisconsin, Madison WI, United States of America

174: Fakultät für Physik und Astronomie, Julius-Maximilians-Universität, Würzburg, Germany

175: Fachbereich C Physik, Bergische Universität Wuppertal, Wuppertal, Germany

176: Department of Physics, Yale University, New Haven CT, United States of America

177: Yerevan Physics Institute, Yerevan, Armenia

178: Domaine scientifique de la Doua, Centre de Calcul CNRS/IN2P3, Villeurbanne Cedex, France

${ }^{179}$ : Faculty of Science, Hiroshima University, Hiroshima, Japan

$a$ : Also at Laboratorio de Instrumentacao e Fisica Experimental de Particulas - LIP, Lisboa, Portugal

${ }^{b}$ : Also at Faculdade de Ciencias and CFNUL, Universidade de Lisboa, Lisboa, Portugal

${ }^{c}$ : Also at Particle Physics Department, Rutherford Appleton Laboratory, Didcot, United Kingdom 
${ }^{d}$ : Also at TRIUMF, Vancouver BC, Canada

$e$ : Also at Department of Physics, California State University, Fresno CA, United States of America

${ }^{f}$ : Also at Novosibirsk State University, Novosibirsk, Russia

${ }^{g}$ : Also at Fermilab, Batavia IL, United States of America

${ }^{h}$ : Also at Department of Physics, University of Coimbra, Coimbra, Portugal

${ }^{i}$ : Also at Università di Napoli Parthenope, Napoli, Italy

${ }^{j}$ : Also at Institute of Particle Physics (IPP), Canada

${ }^{k}$ : Also at Department of Physics, Middle East Technical University, Ankara, Turkey

${ }^{l}$ : Also at Louisiana Tech University, Ruston LA, United States of America

$m$ : Also at Department of Physics and Astronomy, University College London, London, United Kingdom

n: Also at Group of Particle Physics, University of Montreal, Montreal QC, Canada

${ }^{\circ}$ : Also at Department of Physics, University of Cape Town, Cape Town, South Africa

$p$ : Also at Institute of Physics, Azerbaijan Academy of Sciences, Baku, Azerbaijan

q: Also at Institut für Experimentalphysik, Universität Hamburg, Hamburg, Germany

$r$ : Also at Manhattan College, New York NY, United States of America

$s$ : Also at School of Physics, Shandong University, Shandong, China

${ }^{t}$ : Also at CPPM, Aix-Marseille Université and CNRS/IN2P3, Marseille, France

${ }^{u}$ : Also at School of Physics and Engineering, Sun Yat-sen University, Guanzhou, China

$v$ : Also at Academia Sinica Grid Computing, Institute of Physics, Academia Sinica, Taipei, Taiwan

w: Also at DSM/IRFU (Institut de Recherches sur les Lois Fondamentales de l'Univers), CEA Saclay (Commissariat a l'Energie Atomique), Gif-sur-Yvette, France

x: Also at Section de Physique, Université de Genève, Geneva, Switzerland

${ }^{y}$ : Also at Departamento de Fisica, Universidade de Minho, Braga, Portugal

z: Also at Department of Physics and Astronomy, University of South Carolina, Columbia SC, United States of America

${ }^{a a}$ : Also at Institute for Particle and Nuclear Physics, Wigner Research Centre for Physics, Budapest, Hungary

${ }^{a b}$ : Also at California Institute of Technology, Pasadena CA, United States of America

${ }^{a c}$ : Also at Institute of Physics, Jagiellonian University, Krakow, Poland

${ }^{a d}$ : Also at LAL, Univ. Paris-Sud and CNRS/IN2P3, Orsay, France

${ }^{a e}$ : Also at Department of Physics and Astronomy, University of Sheffield, Sheffield, United Kingdom

af : Also at Department of Physics, Oxford University, Oxford, United Kingdom

${ }^{a g}$ : Also at Institute of Physics, Academia Sinica, Taipei, Taiwan

${ }^{a h}$ : Also at Department of Physics, The University of Michigan, Ann Arbor MI, United States of America

ai: Also at Laboratoire de Physique Nucléaire et de Hautes Energies, UPMC and Université Paris-Diderot and CNRS/IN2P3, Paris, France

*: Deceased 